\title{
Monitoring the late events of translation initiation in real-time
}

\author{
Dissertation \\ for the award of the degree \\ "Doctor of Philosophy" (Ph.D.) \\ in the IMPRS Molecular Biology program \\ at the Georg-August University Göttingen \\ Faculty of Biology
}

submitted by

Akanksha Goyal

from New Delhi, India

Göttingen, 2015 
Members of the Thesis Committee

Prof. Marina Rodnina

$\left(1^{\text {st }}\right.$ reviewer)

Prof. Heike Krebber

$\left(2^{\text {nd }}\right.$ reviewer)

Prof. Heinz Neumann
Max Planck Institute for Biophysical Chemistry

Göttingen, Germany

Institute for Microbiology and Genetics

Göttingen, Germany

Göttingen Center for Molecular Biosciences (GZMB)

Göttingen, Germany

\section{Further members of the Examination Board}

$\begin{array}{ll}\text { Prof. Wolfgang Wintermeyer } & \begin{array}{l}\text { Max Planck Institute for Biophysical Chemistry } \\ \text { Göttingen, Germany }\end{array} \\ \text { Prof. Markus Bohnsack } & \begin{array}{l}\text { Universitätsmedizin Göttingen } \\ \text { Institute for Molecular Biology } \\ \text { Göttingen, Germany }\end{array} \\ \text { Prof. Ralf Ficner } & \text { Institute for Microbiology and Genetics } \\ & \text { Göttingen, Germany }\end{array}$

Date of the oral examination: $30^{\text {th }}$ November, 2015 


\section{AFFIDAVIT}

I hereby declare that the presented thesis entitled "Monitoring the late events of translation initiation in real-time" has been written independently and with no other sources and aids than quoted.

Gottingen, $30^{\text {th }}$ September 2015 


\section{TABLE OF CONTENTS}

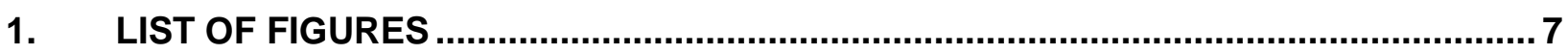

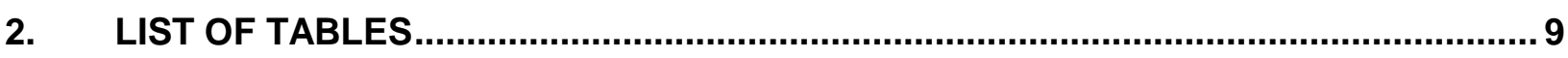

3. ABSTRACT 10

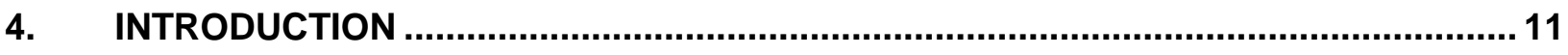

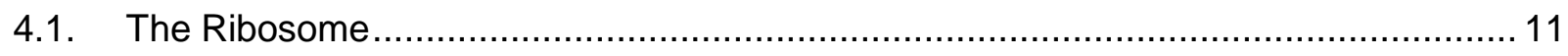

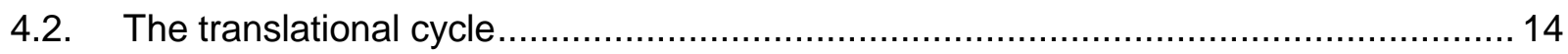

4.3. Key players involved in bacterial translation initiation ......................................... 16

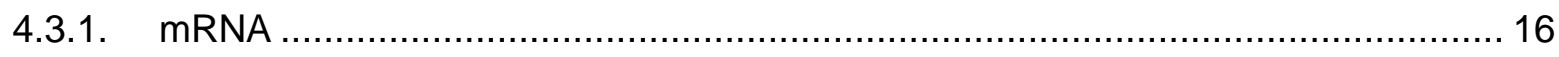

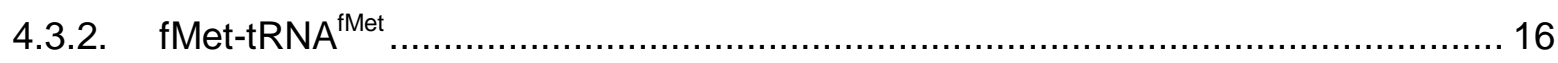

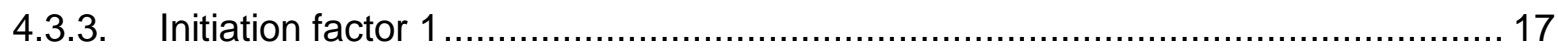

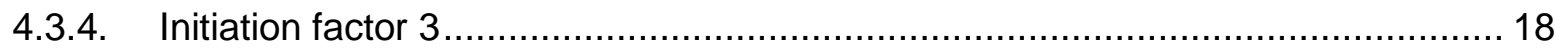

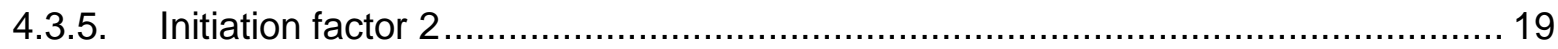

4.4. Translation initiation - kinetic, thermodynamic and structural insights ........................22

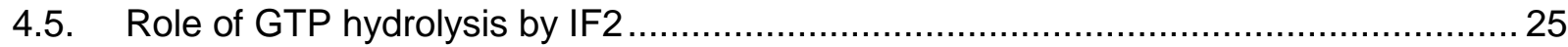

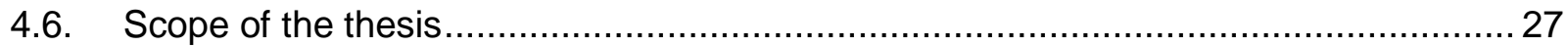

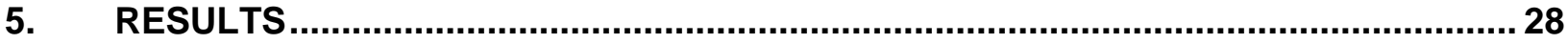

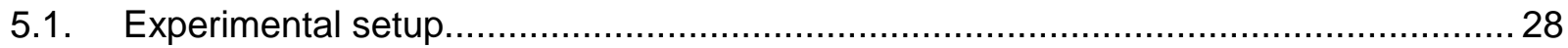

5.2. Screening the activity of translation initiation components ..................................... 30

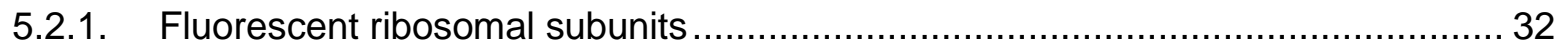

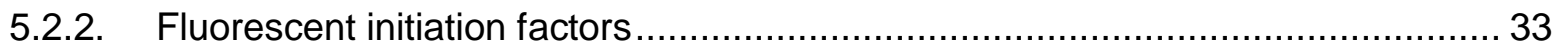

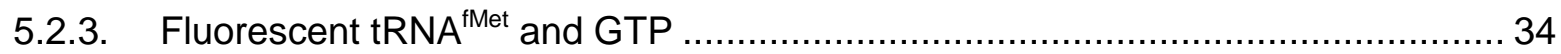

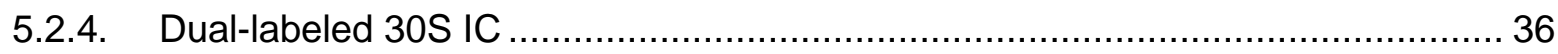

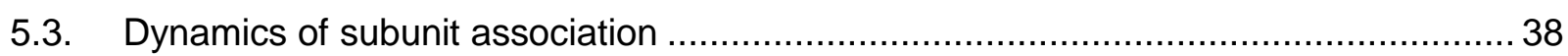

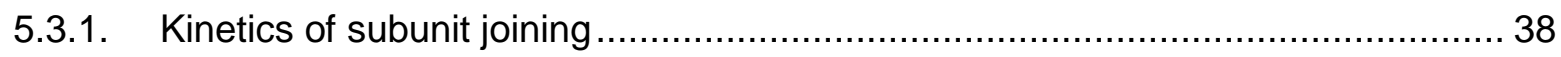

5.3.2. Effect of different GTP analogs on subunit joining .......................................... 39

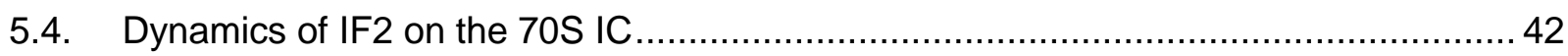

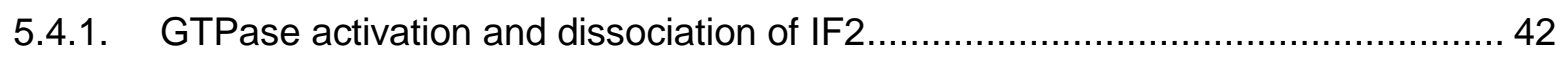

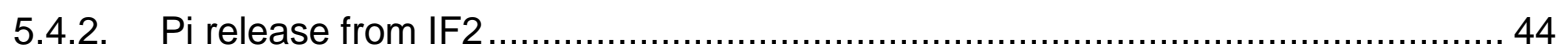

5.4.3. Release of fMet-tRNA ${ }^{\text {fMet }}$ from the C2-domain of IF2 ....................................... 45

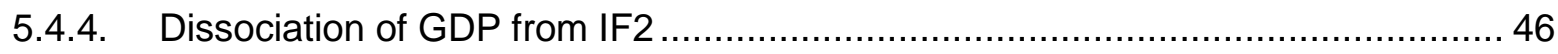

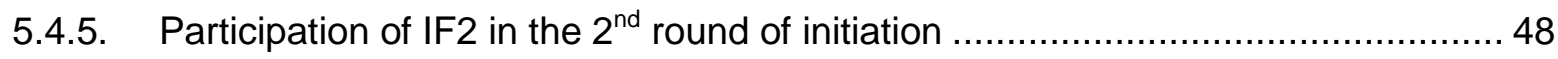

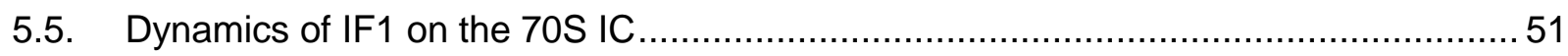


5.5.1. Change in IF1 environment after subunit joining ........................................... 51

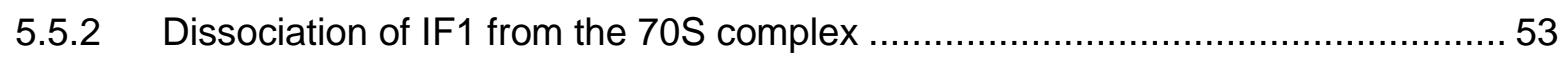

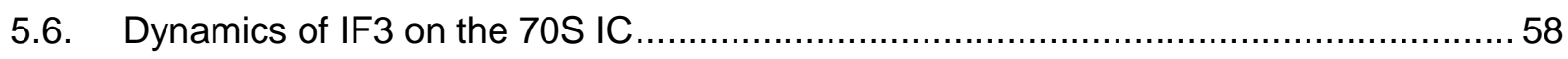

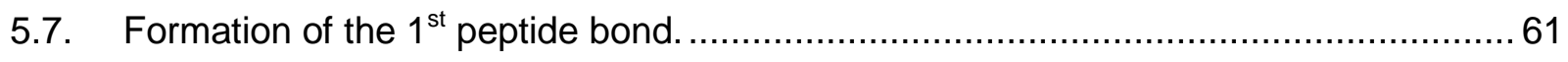

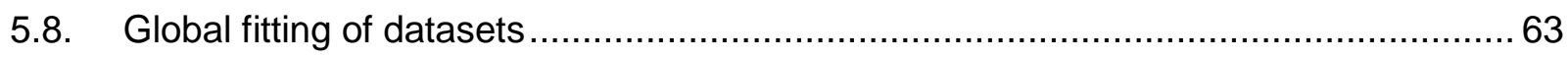

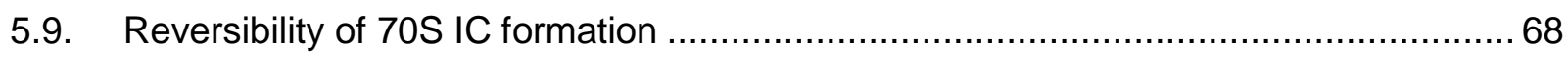

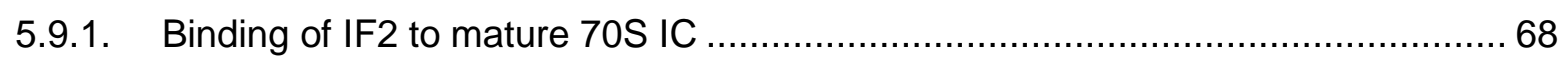

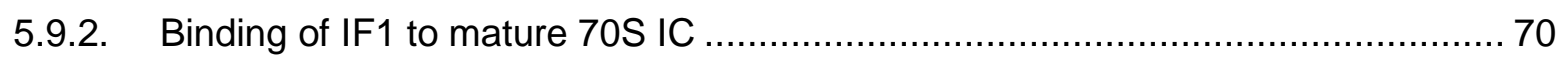

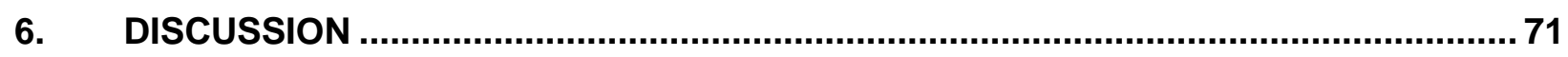

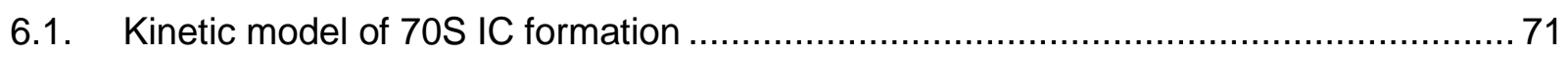

6.2. Role of GTP hydrolysis in maturation of the 70 S complex …................................. 74

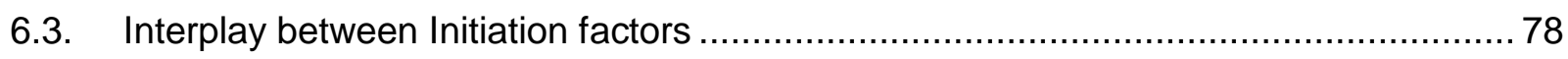

6.4. Comparisons with eukaryotic translation initiation and future perspectives ................. 81

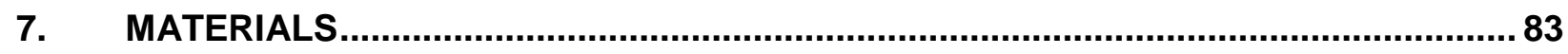

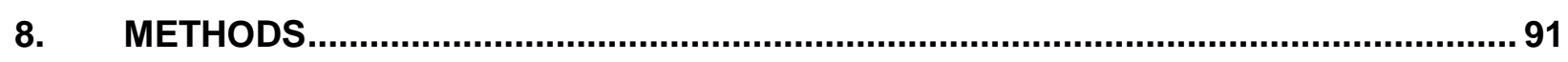

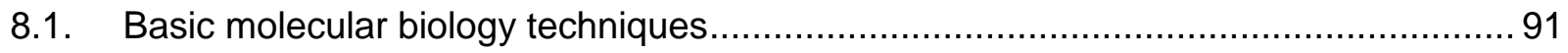

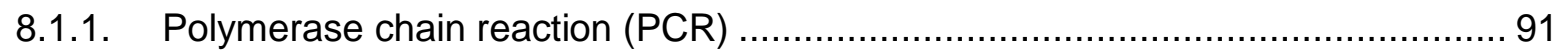

8.1.2. Transformation and plasmid isolation ........................................................... 92

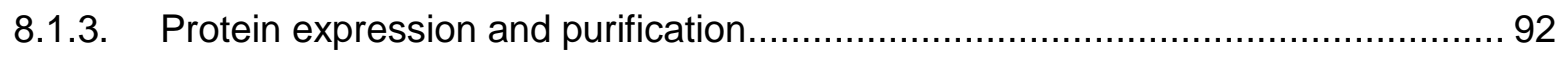

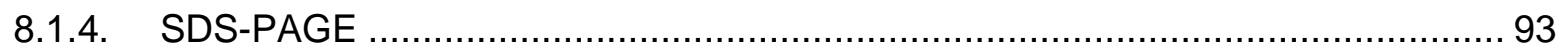

8.2. Preparation of purified translation initiation components ............................................ 93

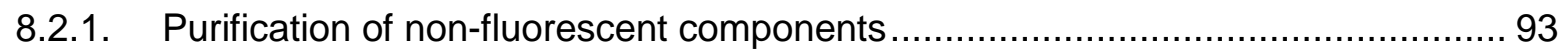

8.2.2. Preparation of fluorescence-labeled components ........................................... 94

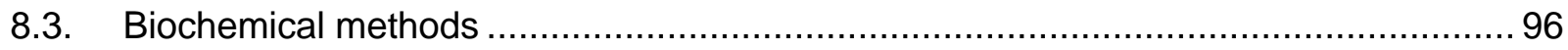

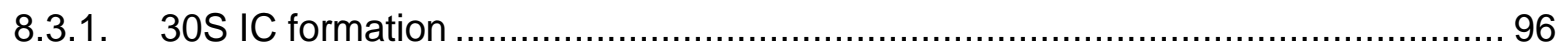

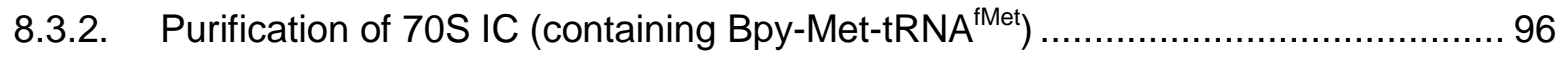

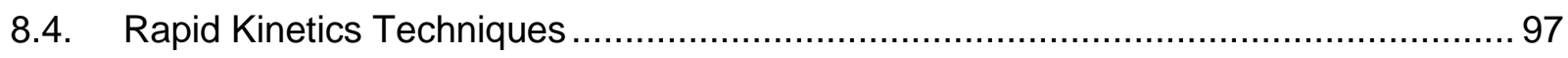

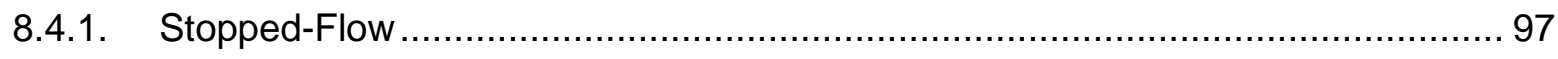

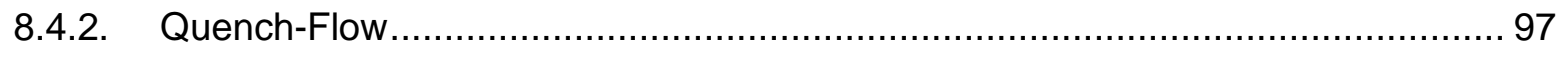

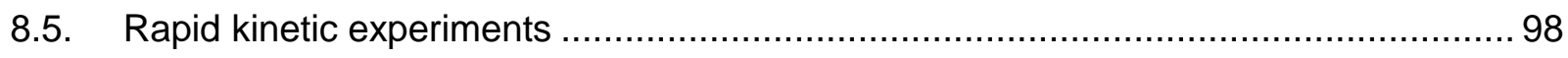

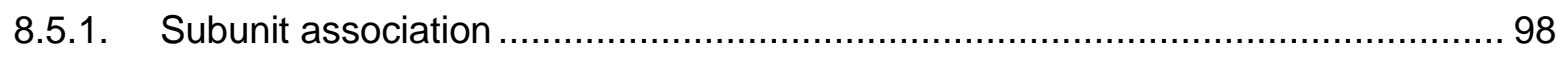

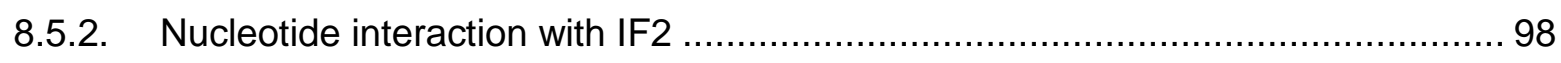

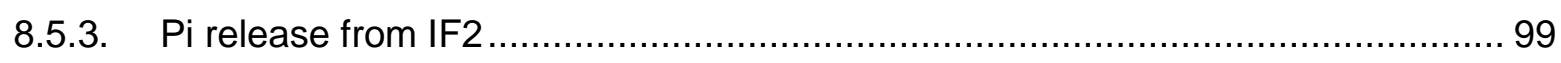

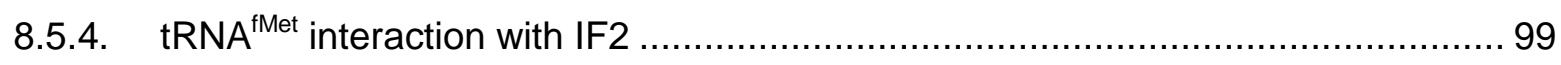


8.5.5. IF2 interaction with the 30 S subunit....................................................... 99

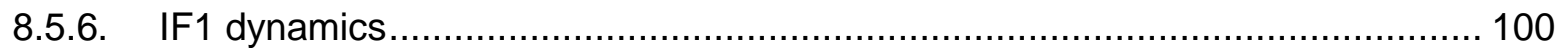

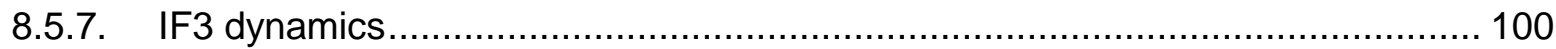

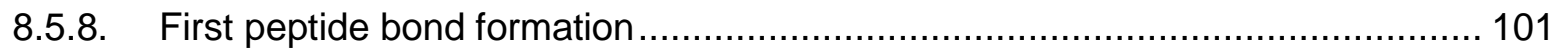

8.5.9. (Re)-association of IF1 and IF2 with mature 70S IC .................................. 101

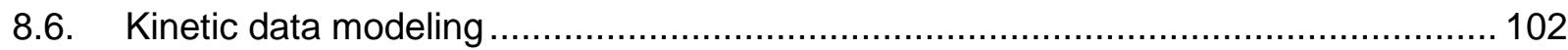

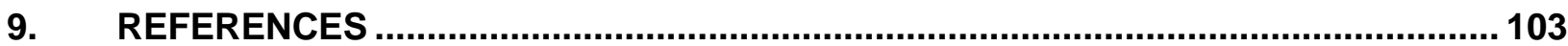

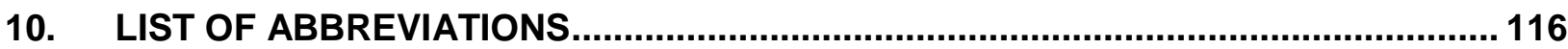

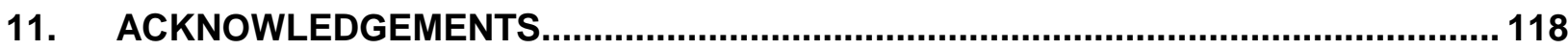

12. Curriculum Vitae ..................................................................................................... 120 


\section{LIST OF FIGURES}

Figure 1. Structure of the ribosome

Figure 2. Translation cycle in bacteria

Figure 3. Interactions of $\mathrm{PMet}^{-\mathrm{tRNA}} \mathrm{A}^{\mathrm{fMet}}$ on the $30 \mathrm{~S} \mathrm{IC}$

Figure 4. Domain structure of IF2 and its interactions on the 30S IC

Figure 5. Schematic of translation initiation in bacteria

Figure 6. Role of translation initiation components in promoting subunit association

Figure 7. Effect of fluorescence-labeled ribosomal subunits on subunit association

Figure 8. Effect of fluorescence-labeled IFs on subunit association

Figure 9. Effect of fluorescence-labeled tRNA ${ }^{\mathrm{fMet}}$ and GTP on subunit association

Figure 10. Effect of dual-labeled $30 \mathrm{~S} \mathrm{IC} \mathrm{on} \mathrm{subunit} \mathrm{association}$

Figure 11. $50 \mathrm{~S}$ subunit association with the $30 \mathrm{~S} \mathrm{IC}$

Figure 12. Effect of different guanosine nucleotide analogs on subunit association

Figure 13. GTPase activation and dissociation of the IF2 during 70S IC formation

Figure 14. Pi release from IF2 on the 70S IC.

Figure 15. Binding and dissociation kinetics of Bpy-Met-tRNA ${ }^{\text {fMet }}$ on the $30 \mathrm{~S} \mathrm{IC}$

Figure 16. Release of tRNA ${ }^{\text {fMet }}$ from IF2 during $70 \mathrm{~S} \mathrm{IC} \mathrm{formation}$

Figure 17. Binding and dissociation kinetics of Bpy-GTP and Bpy-GDP

Figure 18. Release of GDP from IF2 during 70S IC formation

Figure 19. Binding and dissociation kinetics of IF2 $2_{757}($ Alx555) on the $30 \mathrm{~S} \mathrm{IC}$

Figure 20. IF2 binding to $30 \mathrm{~S}$ PIC during the $2^{\text {nd }}$ round of initiation

Figure 21. Binding and dissociation kinetics of $\mathrm{IF}_{4}(\mathrm{Al} \times 555)$ on the $30 \mathrm{~S} \mathrm{IC}$

Figure 22. Change in IF1 environment during $70 \mathrm{~S} \mathrm{IC} \mathrm{formation}$

Figure 23. Binding and dissociation kinetics of $\mathrm{IF}_{1}{ }_{4}$ (Atto540Q) on the $30 \mathrm{~S}_{\mathrm{S} 13}(\mathrm{Al} \times 488) \mathrm{IC}$

Figure 24. Dissociation of IF1 during $70 \mathrm{~S} \mathrm{IC} \mathrm{formation}$

Figure 25. Efforts to uncouple IF1 dissociation from GTP hydrolysis

Figure 26. Effect of non-cognate start codons on IF1 dissociation

Figure 27. Binding and dissociation kinetics of $\mathrm{IF}_{166}(\mathrm{Al} \times 488)$ on the $30 \mathrm{~S} \mathrm{IC} \mathrm{containing}$ IF2 ${ }_{757}$ (Atto540Q) IC

Figure 28. Dissociation of IF3 during 70S IC formation

Figure 29. Formation of fMet-Phe dipeptide

Figure 30. Statistical analysis of global fitting of datasets using KinTek Explorer 
Figure 31. Binding of IF2 to mature 70S IC in the presence of pre- and post-hydrolysis state GTP analogs

Figure 32. Binding of IF1 to mature 70S IC in the presence of GTPYS

Figure 33. Detailed kinetic scheme of late events in bacterial translation initiation

Figure 34. Direct comparison of reaction time courses during 70S IC formation 


\section{LIST OF TABLES}

Table 1. Summary of apparent rate constants of reactions during 70S IC formation

Table 2. Summary of elemental rate constants of reactions during 70 S IC formation

Table $3 . \quad$ Chemicals

Table 4. Fluorophores and radioactive compounds

Table 5. Nucleotides

Table 6. Kits

Table 7. Plasmids

Table 8. DNA primers

Table 9. mRNA

Table 10. Enzymes

Table 11. Buffers and solutions

Table 12. Cell strains

Table 13. Cell culture media

Table 14. Chromatographic columns

Table 15. Instruments

Table 16. Other materials

Table 17. Softwares

Table 18. Conditions used for standard PCR reactions

Table 19. PCR conditions used for Site-directed mutagenesis

Table 20. Preparation of SDS gels 


\section{ABSTRACT}

In bacteria, translation initiation is promoted by three initiation factors (IF1, IF2 and IF3) and entails three distinct steps. First, the small ribosomal subunit (30S) recruits the initiation factors, initiator tRNA (fMet-tRNA ${ }^{\mathrm{fMet}}$ ), mRNA and GTP to form a $30 \mathrm{~S}$ pre-initiation complex (IC). During the next step, the complex matures into a $30 \mathrm{~S} \mathrm{IC} \mathrm{upon} \mathrm{start} \mathrm{codon-anticodon}$ recognition between the mRNA and fMet-tRNA ${ }^{\mathrm{fMet}}$. Finally, the large ribosomal subunit (50S) associates with the 30 S IC giving rise to a 70 S IC which can participate in the first peptide bond formation. Here, the timing and control of reactions that occur after subunit joining are studied with the help of rapid kinetic techniques and fluorescence-labeled translation components. A detailed kinetic model, based on global fitting of time courses, obtained with ten different reporters, is presented. The interplay between IF1 and IF3, as well as the role of IF2-dependent GTP hydrolysis in promoting 70S IC formation is studied. We observe that IF1 and IF3 together affect the rate of subunit joining, but not the following steps of $70 \mathrm{~S}$ IC maturation. GTP hydrolysis does not regulate IF3 dynamics but is required for the efficient dissociation of fMet-tRNA ${ }^{\mathrm{fMet}}$ from IF2. The absence of GTP hydrolysis also prevents the dissociation of IF1 and IF2 from the 70 S ribosome and the equilibrium is shifted towards a stable 70S-IF1-IF2-mRNA-fMet-tRNA ${ }^{\text {fMet }}$ complex, suggesting that GTP hydrolysis guides the irreversible transition of the $70 S$ complex into an elongation-competent state. 


\section{INTRODUCTION}

All life forms depend on critical cellular processes which are carried out by a myriad of proteins. Proteins are linear polymers consisting of amino acids whose sequence is encoded in the messenger RNA (mRNA). Protein synthesis is a fundamental process during which the coding sequence of the mRNA is translated by the ribosome into a corresponding polypeptide chain. The ribosome performs this task with the help of several translation factors, by recruiting the transfer RNA (tRNA) substrate which carries the respective amino acid. The bacterial translation apparatus is an important target for antibiotics, and compensatory mutations in the ribosome or translation factors lead to the generation of resistant strains. In mammals, defects in any part of the translation apparatus, including but not limited to mRNA, tRNA, tRNA modifying enzymes, translation factors or the ribosomal proteins, have been implicated in neurodegenerative and autoimmune diseases as well as tumorigenesis. In addition, several viruses have evolved to competently hijack the translation machinery of their host to favor their own propagation. Due to its significance in health and disease, the translation process warrants in-depth study, as the resulting mechanistic or structural insights may help generate novel therapeutic agents.

\subsection{The Ribosome}

Ribosomes are large macromolecular complexes (2.5-4.3 MDa) responsible for protein synthesis inside the cell. Bacterial ribosomes consist of roughly $60 \%$ RNA and $40 \%$ proteins. The interior of the ribosome is composed mostly of ribosomal RNA (rRNA), while the ribosomal proteins are found in the exterior of the particle, nestled between the rRNA elements (Ban et al., 2000). Bacterial ribosomes have a sedimentation coefficient of $70 \mathrm{~S}$ (Svedberg units) and consist of a small (30S) and a large (50S) subunit. The $30 \mathrm{~S}$ subunit consists of 21 polypeptide chains (designated S1 - S21) surrounding a 16S rRNA ( 1500 nts). The $30 \mathrm{~S}$ subunit binds to the mRNA template and ensures faithful decoding of mRNA codons. The 50S subunit consists of the 23S rRNA ( 2900 nts), 5S rRNA ( 120 nts), and 34 ribosomal proteins (designated L1 - L34). It holds two important catalytic centers - the peptidyl transferase center (PTC) which catalyzes the peptide bond formation, and the GTPase-activating center that stimulates the GTPase activity of the translation factors. The two subunits interact with each other via inter-subunit bridges formed by proteins and rRNA elements at the subunit interface. The ribosome contains three binding sites for tRNA (Fig. 1): the aminoacyl (A) site receives the incoming aminoacyl tRNA, the peptidyl (P) site holds 
the tRNA bound to the polypeptide chain and the exit (E) site guides the release of the deacylated tRNA from the ribosome.

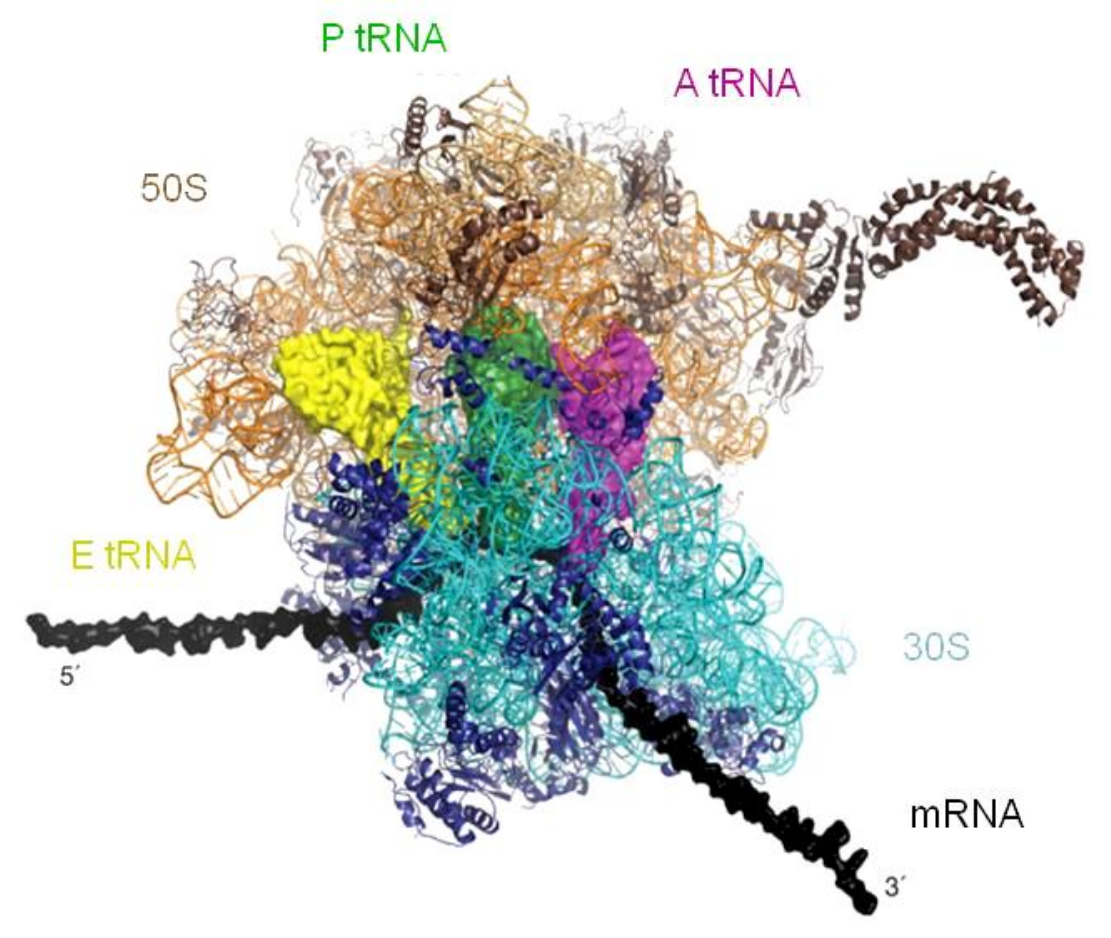

Figure 1. Structure of the ribosome. Crystal structure of the 705 ribosome complex with the mRNA and tRNAs (Schmeing and Ramakrishnan, 2009). The 30S and 50S subunits are depicted in blue and orange, respectively. The tRNAs in the $A, P$, and $E$ sites are shown in magenta, green, and yellow, respectively. The mRNA forms codon-anticodon interactions with the tRNAs in the decoding centre of the 30 S subunit.

Early structural information about the bacterial ribosome was derived mainly from lowresolution cryo-electron microscopy, cross-linking and chemical footprinting studies (reviewed in (Fraser and Doudna, 2007; Green et al., 1998)). Advances in the field of crystallization, data collection and computation facilitated the determination of several crystal structures (Ban et al., 2000; Harms et al., 2001; Schluenzen et al., 2000; Schuwirth et al., 2005; Selmer et al., 2006; Wimberly et al., 2000; Yusupov et al., 2001; Yusupova et al., 2001) and gave valuable insights into the architecture of the ribosome. Recent highresolution cryo-EM structures (Bai et al., 2013; Fischer et al., 2015; Li et al., 2015) have provided a dynamic picture of conformational states occupied by the ribosome during translation. Biochemical and genetic approaches have been used to dissect different stages of the translation pathway, as well as to study the importance of various ribosomal proteins, rRNA, translation factors and tRNAs. Rapid kinetic and single-molecule fluorescence techniques have allowed us to monitor conformational changes and ligand interactions on 
the ribosome and have given rise to detailed models of translation in bacteria (Blanchard, 2009; Blanchard et al., 2004; Milon et al., 2008; Milon et al., 2012; Pape et al., 1998; Peske et al., 2005; Petrov et al., 2012; Rodnina et al., 2006; Rodnina et al., 1997; Savelsbergh et al., 2003; Shoji et al., 2009; Wintermeyer et al., 2004). Complementing the advances made in solving the ribosome structures by X-ray crystallography and cryo-EM, the biochemical and biophysical techniques have provided a comprehensive mechanistic insight into the dynamics of the ribosome and translation factors. 


\subsection{The translational cycle}

Translation by the ribosome occurs in four major stages: initiation, elongation, termination, and ribosome recycling (reviewed in (Ramakrishnan, 2002)) (Fig. 2). Several translation factors termed initiation factors (IF), elongation factors (EF) and release factors (RF) facilitate each step.

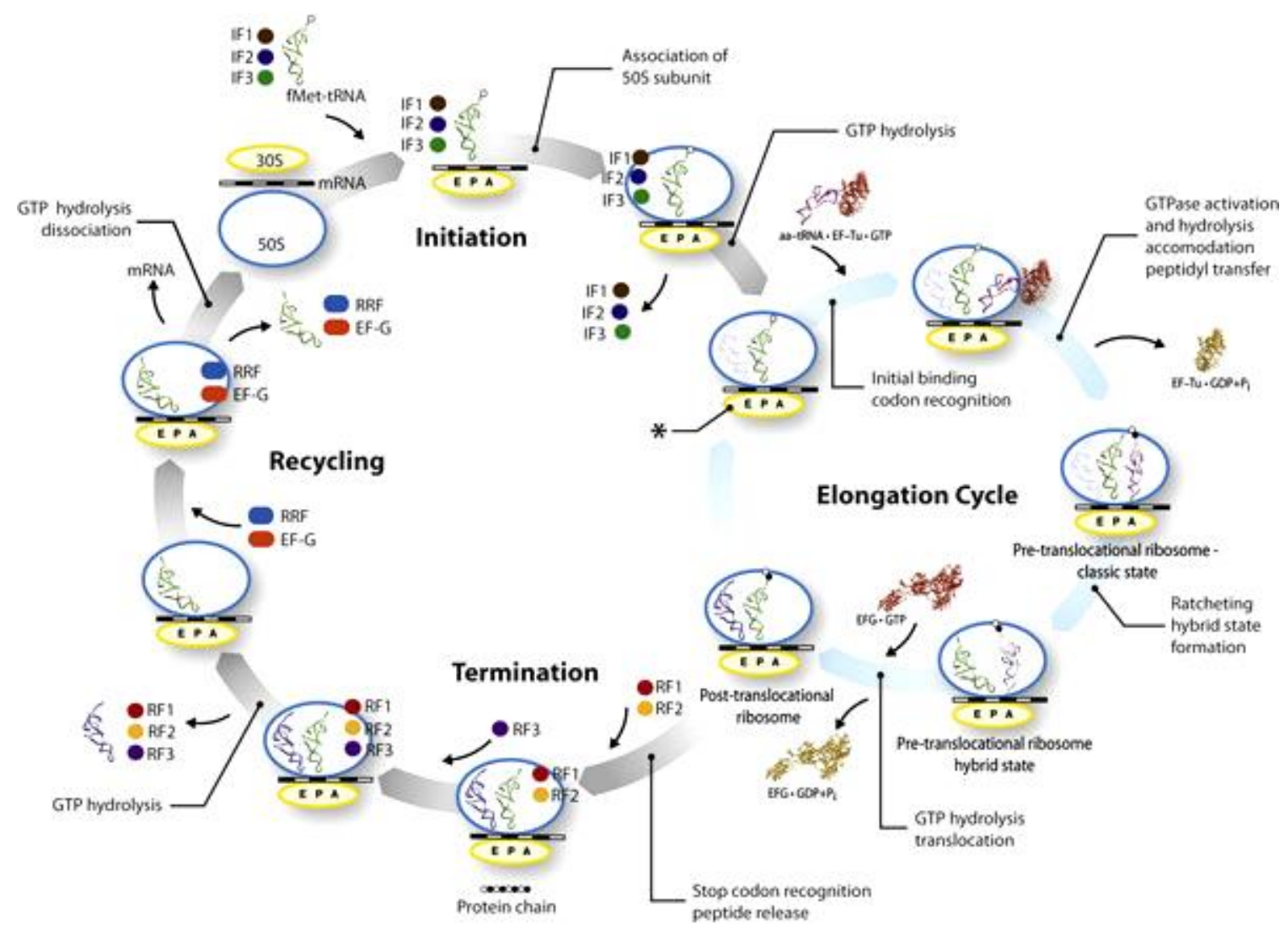

Figure 2. Translation cycle in bacteria. Translation involves four stages - initiation, elongation, termination and ribosomal recycling. During translation initiation, the $30 \mathrm{~S}$ subunit binds IF1, IF2-GTP, IF3, initiator tRNA (fMet-tRNA ${ }^{\mathrm{fMet}}$ ) and mRNA. The $50 \mathrm{~S}$ subunit joins this complex, IF2 hydrolyses GTP and all IFs are released. During elongation, EF-Tu and EF-G facilitate lengthening of the polypeptide chain until a stop codon is encountered (see text for details). RF1, RF2, and RF3 and recycling factors (RRF, EF-G) promote polypeptide release and subunit dissociation allowing the translation machinery to undergo subsequent initiation events (Agirrezabala and Frank, 2010).

During translation initiation, the $30 \mathrm{~S}$ subunit binds the mRNA, IF1, IF2 (a GTP-binding protein), IF3 and fMet-tRNA ${ }^{\text {fMet }}$ to form a $30 \mathrm{~S}$ pre-initiation complex (30S PIC). The anticodon of MMet-tRNA $^{\text {fMet }}$ base-pairs with the AUG start codon of the mRNA and the ribosomal complex matures into a $30 \mathrm{~S}$ initiation complex (30S IC). Upon $50 \mathrm{~S}$ subunit joining, GTP 
hydrolysis by IF2 is triggered, fMet-tRNA ${ }^{\mathrm{fMet}}$ enters the canonical P/P site and all IFs are released to give rise to a mature $70 \mathrm{~S} \mathrm{IC} \mathrm{which} \mathrm{can} \mathrm{partake} \mathrm{in} \mathrm{peptide} \mathrm{bond} \mathrm{formation.}$

In the next phase of translation elongation, EF-Tu (a GTP-binding protein) carries an aminoacyl-tRNA (aa-tRNA ${ }^{\text {aa }}$ ) to the A site of the ribosome. After correct codon-anticodon interaction is established between the mRNA and the aa-tRNA ${ }^{\text {aa }}$, in a process known as decoding, EF-Tu hydrolyses GTP and dissociates from the ribosome. The aa-tRNA ${ }^{\text {aa }}$ is accommodated into the ribosomal A site and the peptidyl transferase center (PTC) on the $50 S$ subunit catalyzes peptide bond formation between the two tRNAs present in the $A$ and $P$ sites. The ribosomal subunits rotate with respect to each other, triggering the tRNAs to enter hybrid $P / E$ and $A / P$ sites, where the anticodon loops of the tRNAs remain in the $P$ and $A$ site of the $30 \mathrm{~S}$ subunit and the respective acceptor stems move into the $E$ and $P$ sites of the $50 \mathrm{~S}$ subunit. Thereafter, in a process known as translocation, EF-G (a GTP-binding protein) binds to the ribosome and promotes the movement of the mRNA and tRNAs on the $30 \mathrm{~S}$ subunit from the $P$ and $A$ sites to the $E$ site and $P$ sites, respectively. Translocation is coupled to GTP hydrolysis by EF-G. Subsequently, EF-G and the E-site deacylated tRNA dissociate from the ribosome. Multiple rounds of decoding and translocation take place until a stop codon (UAA, UAG or UGA) enters the A site of the ribosome.

Stop codons are recognized by class I release factors RF1 and RF2 which enter the A site and promote hydrolysis of the aminoacyl ester bond of the P site peptidyl tRNA, resulting in the release of the nascent peptide chain from the ribosome. Another release factor, RF3, facilitates dissociation of RF1/2 from the ribosome. GTP hydrolysis by RF3 promotes its own release from the complex. Finally, the ribosomal subunits are separated to take part in further rounds of translation. The ribosomal release factor (RRF) perturbs the bridging interactions between the subunits while EF-G promotes subunit dissociation. The mRNA is released and IF3 binds the 30 S subunit to stabilize the subunit dissociation event and promote the release of deacylated tRNA.

In the following text, we will focus on translation initiation in bacteria. First, the different components which participate in the process are introduced. The current understanding of the initiation mechanism is discussed and finally, a brief summary describing the aim of the thesis is provided. 


\subsection{Key players involved in bacterial translation initiation}

\subsection{1. mRNA}

Bacterial mRNAs are usually polycistronic and harbor a translation initiation region (TIR) spanning 30-35 nts which affects the efficiency of ribosome recruitment (Dreyfus, 1988). The highly efficient TIR is characterized by several features such as (i) the AUG start codon (ii) presence of the Shine-Dalgarno (SD) sequence (iii) low thermodynamic stability of RNA folding around the initiation site, and (iv) presence of $A / U$ rich sequence elements in the 5 , mRNA leader, the average length of which is $\leq 40 \mathrm{nts}$.

$A U G$ is the most frequently used start codon in bacteria, although GUG and UUG are also commonly observed ( $\mathrm{Ma}$ et al., 2002). 40\% of all bacterial mRNAs harbor an SD sequence (GGAGG) upstream of the initiation codon (Shine and Dalgarno, 1974). The optimal spacing between the SD sequence and the start codon for E. coli mRNAs is 5-8 bases (Chen et al., 1994). The SD sequence of the mRNA base pairs with the 3 ' end of 165 rRNA (anti-SD sequence) in the $30 \mathrm{~S}$ subunit, thereby positioning the start codon in the $\mathrm{P}$ site of the $30 \mathrm{~S}$ subunit (Shine and Dalgarno, 1974).

As noted above, a large number of mRNAs do not have an SD sequence. These mRNAs, divided into non-SD led and leaderless mRNA, do not follow the traditional translation initiation pathway (Grill et al., 2001; Moll et al., 2004). In this thesis, only SD-containing mRNAs have been utilized to study the canonical pathway of bacterial translation initiation.

\subsection{2. fMet-tRNA ${ }^{\mathrm{fMet}}$}

In $E$. coli, there are four copies of the $\operatorname{tRNA}^{\mathrm{fMet}}$ gene; metV, metW, and metY and met $Z$ (Ikemura and Ozeki, 1977). The 3' end of fMet-tRNA ${ }^{\text {fMet }}$ carries formyl-methionine, which will be the first amino acid incorporated into the translated polypeptide. Formylation favors the binding of IMet-tRNA $^{\text {fMet }}$ to the C-terminal domain of IF2 (the C2 domain) (Fig. 3A) which recruits it to the 30S subunit (reviewed in (Laursen et al., 2005)). On the 30S IC, the elbow of fMet-tRNA $^{\text {fMet }}$ contacts the N-terminal domain of IF3 (IF3N) (Julian et al., 2011) (Fig. 3B), while the anticodon loop of fMet-tRNA ${ }^{\mathrm{fMet}}$ base pairs with the start codon of the mRNA in the ribosomal $\mathrm{P}$ site. 
(A)

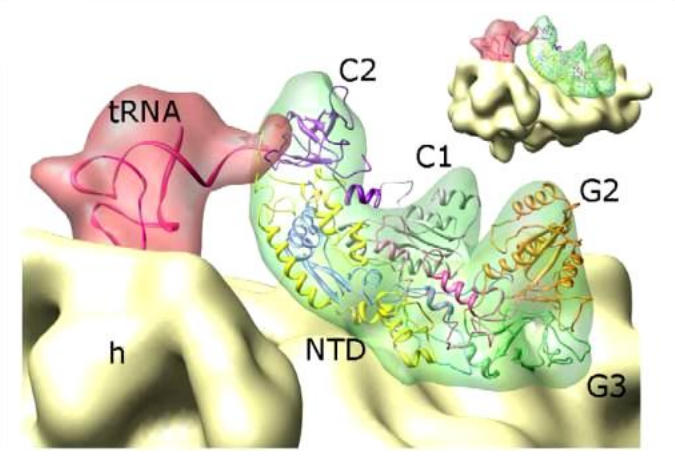

(B)

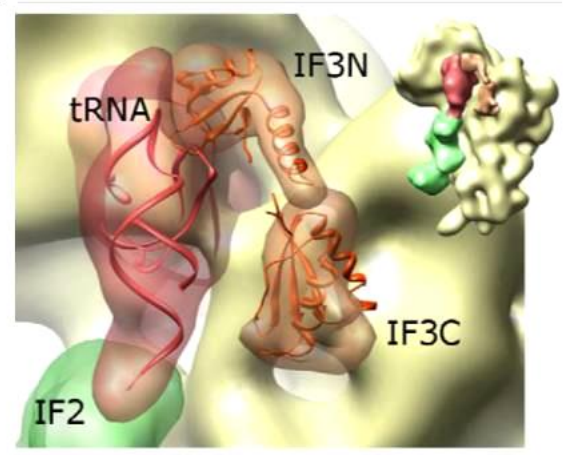

Figure 3. Interactions of fMet-tRNA ${ }^{\mathrm{fMet}}$ on the 30S IC. (A) Cryo-EM reconstruction of fMettRNA ${ }^{\text {tMet }}$ IF2 complex on the 30S IC. The 30S subunit is shown in yellow, the densities for fMet-tRNA $^{\text {fMet }}$ and IF2 in pink and green, respectively. The different sub-domains of IF2 are shown as ribbons - N1 (yellow), N2 (blue), G1 (pink), G2 (orange), G3 (green), C1 (grey), and C2 (purple). (B) Cryo-EM reconstruction of the interaction between fMet-tRNA ${ }^{\text {fMet }}$ and IF3N on the 30S IC. Density for IF3 is shown in orange. Thumbnails show the orientation of the 30 S subunit (Julian et al., 2011).

Cryo-EM structures of the $30 \mathrm{~S}$ and $70 \mathrm{~S}$ initiation complexes have provided insight into fMettRNA $^{\text {fMet }}$ dynamics during IC formation (Allen et al., 2005; Julian et al., 2011; Myasnikov et al., 2005; Simonetti et al., 2008). The positioning of fMet-tRNA $A^{\text {fMet }}$ in the $30 \mathrm{~S} \mathrm{IC} \mathrm{and} \mathrm{the} \mathrm{early}$ stages of the $70 \mathrm{~S}$ complex formed after subunit joining differs from the ribosomal P/P site, where both the anticodon and the acceptor end of the tRNA are placed in the P site of the $30 S$ and $50 S$ subunit, respectively. While the anticodon stem is buried in the P site of the 30 s subunit in both ICs, the position of the CCA-acceptor end changes during maturation of the complex. An intermediate site on the 30S IC was visualized where the acceptor stem was positioned in the supposed space between 50S A and P sites (Julian et al., 2011). In addition, a hybrid site was identified at the early-stage $70 \mathrm{~S}$ complex, which positioned the CCA-stem between the E site and $P$ site of the $50 S$ subunit (Allen et al., 2005). The final accommodation of fMet-tRNA ${ }^{\text {fMet }}$ into the canonical P/P site in the mature $70 S$ IC may require IF2-dependent GTP hydrolysis (Kuechler, 1971; Thach and Thach, 1971) and the dissociation of the fMet-tRNA ${ }^{\mathrm{fMet}}$ CCA-end from the C2-domain of IF2 (Allen et al., 2005; Myasnikov et al., 2005).

\subsubsection{Initiation factor 1}

IF1 is encoded by the infA gene in E. coli. It is a compact $8 \mathrm{kDa}$ protein consisting of 71 amino acids. On the 30 S IC, IF1 binds in the cleft between ribosomal protein S12, the 530 loop and helix 44 of $16 \mathrm{~S}$ rRNA (Carter et al., 2001). It interacts with 16S rRNA of the $30 \mathrm{~S}$ 
subunit by binding to the $A$ site, thereby hindering the entry of aa-tRNAs into the $A$ site during translation initiation (Moazed et al., 1995). IF1 increases the affinity of IF2 and IF3 binding to the $30 \mathrm{~S}$ subunit and modulates mRNA and fMet-tRNA ${ }^{\text {fMet }}$ selection (Boileau et al., 1983; Milon et al., 2012; Surkov et al., 2010). Along with IF3, it acts as an anti-association factor and prevents $50 \mathrm{~S}$ joining by influencing the $30 \mathrm{~S}$ conformation (Grigoriadou et al., 2007b; Milon et al., 2008). Despite several biochemical and genetic studies (Croitoru et al., 2004; Cummings and Hershey, 1994; Milon et al., 2008; Pon and Gualerzi, 1984), little is known about the exact function of IF1 and the timing of its release during $70 \mathrm{~S} \mathrm{IC} \mathrm{formation.} \mathrm{The}$ homologue of IF1 in eukaryotes is elF1A (Kyrpides and Woese, 1998).

\subsubsection{Initiation factor 3}

IF3 is encoded by the infC gene in E. coli. It is a $20 \mathrm{kDa}$ protein built of 180 amino acids. It consists of two domains, the N-terminal (IF3N) and C-terminal domain (IF3C), connected by a flexible lysine-rich linker region (Kycia et al., 1995; Moreau et al., 1997). On the 30S IC, the IF3C domain is placed at the 790 loop of the 16S rRNA and the IF3N domain is positioned near fMet-tRNA ${ }^{\text {fMet }}$ (Dallas and Noller, 2001; Fabbretti et al., 2007; Julian et al., 2011; McCutcheon et al., 1999; Pioletti et al., 2001; Sette et al., 1999; Shapkina et al., 2000). The IF3C domain blocks the binding site for helix 69 of the 23S rRNA on the 50 S subunit, thereby impeding subunit association (Dallas and Noller, 2001; Julian et al., 2011).

The main functions of IF3 are: (i) promoting the dissociation of deacylated tRNA and translated mRNA from the ribosome after recycling by RRF and EF-G (Hirokawa et al., 2002; Karimi et al., 1999; Peske et al., 2005), (ii) preventing the premature association of the ribosomal subunits (Grunberg-Manago et al., 1975), (iii) facilitating adjustment of the mRNA into the 30S P site (La Teana et al., 1995), (iv) discriminating against mRNAs with unfavorable TIRs (Maar et al., 2008; Milon et al., 2008), and (v) monitoring the initiation fidelity by causing preferential dissociation of a non-initiator tRNA during 30S IC formation (Antoun et al., 2006a; Hartz et al., 1990; Meinnel et al., 1999; Sussman et al., 1996). Correct start codon-anticodon recognition between the mRNA and fMet-tRNA ${ }^{\text {fMet }}$ results in destabilization of IF3 binding to the 30S subunit, allowing rapid 50S joining to occur (Milon et al., 2008; Milon et al., 2012). All functions attributed to the full-length IF3 can be performed by the IF3C domain alone. The main function attributed to the IF3N domain is the enhancement of affinity between IF3 and the 30 S subunit (Petrelli et al., 2001). The functional homologue of IF3 in eukaryotes is elF1 (Lomakin et al., 2003). 
The timing of IF3 release from the ribosome has been much debated. On the basis of indirect biochemical studies with an mRNA containing an enhanced non-natural SD sequence, it was suggested that IF3 dissociates before 50S docking (Antoun et al., 2006b), thus freeing the principle inter-subunit bridge B2b which is involved in stable subunit association. However, rapid kinetic (Milon et al., 2008) and single-molecule FRET measurements (Elvekrog and Gonzalez, 2013; MacDougall and Gonzalez, 2015) showed that 30S IC, formed with mRNAs containing a natural SD sequence, can bind the 50S subunit before IF3 is released (Grigoriadou et al., 2007b; Milon et al., 2008; Pon and Gualerzi, 1986). Additionally, results of time-resolved chemical probing indicated that IF3 dissociates from the ribosome in a stepwise manner during, and not before, the formation of 70 S IC (Fabbretti et al., 2007).

\subsubsection{Initiation factor 2}

IF2 is encoded by the infB gene and is the largest IF involved in prokaryotic translation. The homologues of IF2 in archea and eukaryotes are alF5B and elF5B, respectively. In E. coli, it is a $97.3 \mathrm{kDa}$ protein consisting of 890 amino acids. In the past two decades, several bioinformatic, biochemical and structural studies have helped to characterize the structure of IF2 from the archeal organism Methanobacterium thermoautotrophicum, and bacteria such as E. coli, Geobacillus stearothermophilus (G. staerothermophilus) and Thermus thermophilus (T. thermophilus) (Caserta et al., 2010; Caserta et al., 2006; Eiler et al., 2013; Guenneugues et al., 2000; Laursen et al., 2004; Laursen et al., 2003; Meunier et al., 2000; Moreno et al., 1999; Mortensen et al., 1998; Rasmussen et al., 2008; Roll-Mecak et al., 2000; Simonetti et al., 2013; Wienk et al., 2012; Zoldak et al., 2008). IF2 consists of three main structural domains; the least conserved and poorly structured N-terminal domain (NTD is divided into N1 and N2 sub-domains), the nucleotide binding G-domain (divided into subdomains G1, G2 and G3), and the fMet-tRNA ${ }^{\text {fMet }}$ binding C-terminal domain (CTD is divided into $C 1$ and $C 2$ sub-domains) (Fig. 4A). The G2 sub-domain of IF2 is involved in GTP binding, whereas the G3 sub-domain is conserved in all translation GTPases. The C1 subdomain is rich in helices and the C2 sub-domain interacts with the 3' CCA-end of fMettRNA $^{\text {fMet }}$ (Guenneugues et al., 2000; Krafft et al., 2000; Simonetti et al., 2008; Spurio et al., 2000) (Fig. 4B). 
(A)

\begin{tabular}{|l|l|l|ll|l|l|l|l|l|}
\hline $\mathrm{N} 1$ & $\mathrm{~N} 2$ & & $\mathrm{G} 1$ & & $\mathrm{G} 2$ & $\mathrm{G} 3$ & \multicolumn{2}{c|}{ C1 } & \multicolumn{2}{c|}{ C2 } \\
\hline 1
\end{tabular}

(B)

(C)
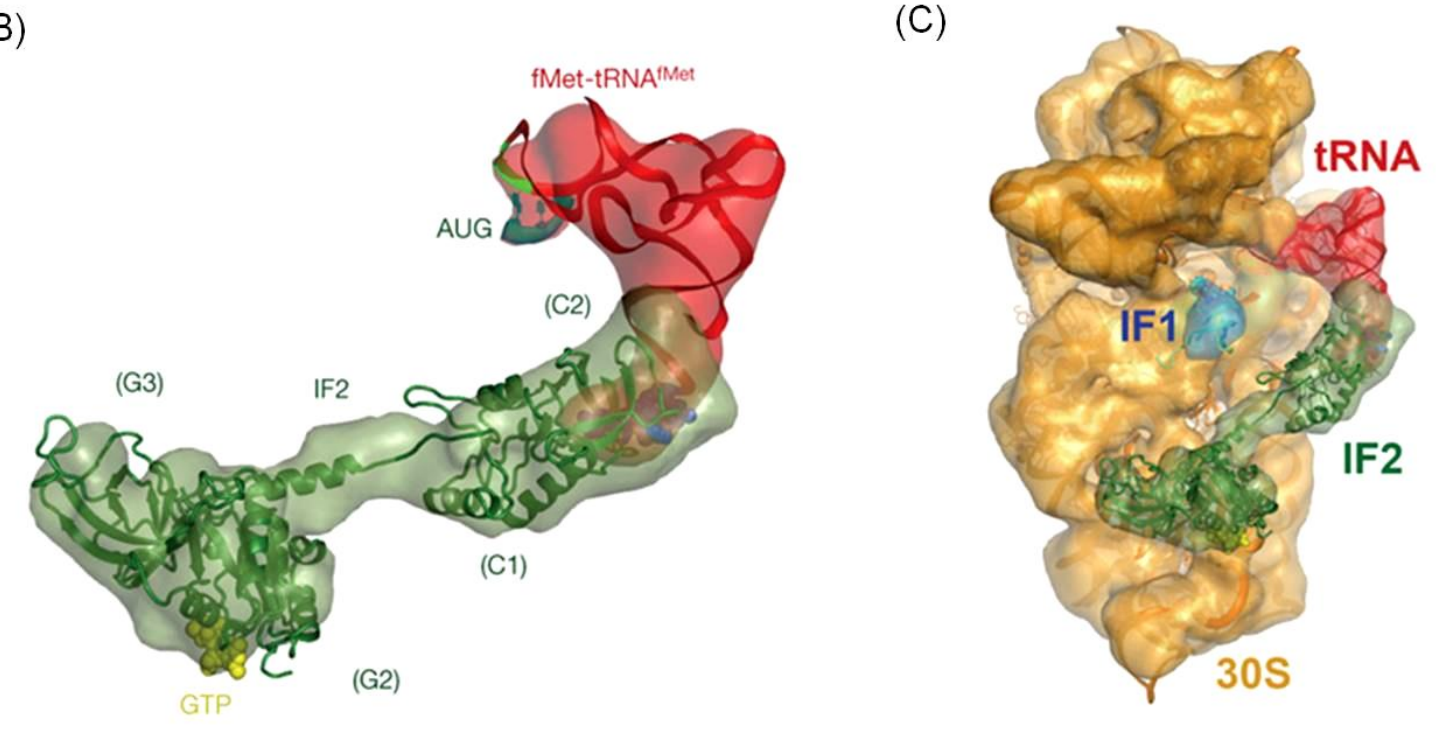

Figure 4. Domain structure of IF2 and its interactions on the 30S IC. (A) Division of IF2 peptide sequence from E. coli into its sub-domains (Caserta et al., 2006). (B) The IF2-fMettRNA $^{\text {tMet }}$ sub-complex. Interaction of IF2 (green) from Thermus thermophilus with the fMettRNA $^{\text {fMet }}$ (red) is shown (Simonetti et al., 2008). GTP (yellow) binds to the G2 domain. The tRNA $^{\text {fMet }}$ anticodon (light green) base pairs with the mRNA start codon (dark green) (C) CryoEM reconstruction of the 30 S IC lacking IF3. Positions of fMet-tRNA ${ }^{\text {fMet }}$ (red), IF1 (blue), and IF2 (green) on the 30S are shown (Simonetti et al., 2008).

Cryo-EM structures of the 30S IC (Fig. 4C) (Julian et al., 2011; Simonetti et al., 2008) and 70 S PIC (Allen et al., 2005; Myasnikov et al., 2005) have provided important insights into IF2 conformational states and its placement on the ribosome. The IF2 N-domain was implicated in binding to the S12 protein of the 30S subunit ((Julian et al., 2011) and references therein) and directly interacts with IF1 (Julian et al., 2011). Domain G2 is oriented towards the sarcinricin loop of the 23S rRNA on the 50S subunit (Allen et al., 2005; La Teana et al., 2001; Myasnikov et al., 2005; Simonetti et al., 2008) and this interaction has been implicated in activation of GTP hydrolysis by IF2 (Beaudry et al., 1979). Domains G3 and C1 interact with the 16S rRNA (Julian et al., 2011; Simonetti et al., 2008) while the C2-domain interacts with fMet-tRNA ${ }^{\text {fMet }}$ which anchors the factor to the $30 \mathrm{~S}$ subunit via the pairing of its anticodon loop with the mRNA start codon (Allen et al., 2005; Guenneugues et al., 2000; Simonetti et al., 2008). The conformational state of IF2 differs depending on the guanine nucleotide (GTP or 
GDP) bound to it. IF2 binds GTP with a relatively low affinity of $40 \mu \mathrm{M}$ off the ribosome, and with a higher binding affinity of $2 \mu \mathrm{M}$ on the ribosome (Antoun et al., 2003). Because the affinity of GTP and GDP binding to IF2 under physiological temperatures is similar (Hauryliuk et al., 2009), and there is no specific nucleotide exchange factor in the cell for IF2, the nucleotide exchange takes place due to a high rate of GDP dissociation and a high cellular concentration of GTP (Milon et al., 2006). It has been postulated that GTP binding by IF2 may serve as a metabolic sensor of the nutritional state of the cell. Under optimal growth conditions, when GTP is abundant, binding of GTP to IF2 promotes translation. Under nutritional stress, the cellular concentration of a signaling molecule ppGpp increases. ppGpp binds to IF2 and renders the factor inactive, thus inhibiting translation (Gualerzi et al., 2001; Milon et al., 2006).

Free IF2 binds fMet-tRNA ${ }^{\text {fMet }}$ with a low affinity (in the micromolar range) forming an unstable complex that dissociates readily ((Milon et al., 2010) and references therein). In its GTP bound form, IF2 recruits fMet-tRNA ${ }^{\text {fMet }}$ to the 30 S subunit with high affinity (in the nanomolar range) (Milon et al., 2010) discriminating against elongator tRNAs and non-formylated MettRNA ${ }^{\text {fMet }}$ (Antoun et al., 2006a; Boelens and Gualerzi, 2002). Binding of both GTP and fMettRNA ${ }^{\text {fMet }}$ confers an 'active' conformation of IF2 on the 30 S subunit (Pavlov et al., 2011; Zorzet et al., 2010) which promotes rapid subunit joining. 30S-bound IF2 interacts with the ribosomal stalk (consisting of ribosomal proteins L7/L12) of the 50 S subunit to promote subunit association (Allen et al., 2005; Helgstrand et al., 2007; Huang et al., 2010; Mandava et al., 2012). The large surface area of IF2 shields negative electrostatic charges of the rRNAs at the interface of the $30 \mathrm{~S}$ and $50 \mathrm{~S}$ subunits, and forms rRNA-protein and proteinprotein bridges with the subunits to stabilize their interaction (Allen et al., 2005; Simonetti et al., 2008). 50 S subunit joining to the 30 S IC triggers rapid hydrolysis of GTP by IF2 into GDP and inorganic phosphate (Pi) (Grigoriadou et al., 2007a; Huang et al., 2010; Tomsic et al., 2000) which may promote the adjustment of fMet-tRNA ${ }^{\text {fMet }}$ into the ribosomal $P$ site and subsequent release of the factor from the $70 \mathrm{~S}$ complex. 


\subsection{Translation initiation - kinetic, thermodynamic and structural insights}

Initiation of protein synthesis establishes the reading frame of the mRNA by positioning the initiator tRNA (fMet-tRNA ${ }^{\mathrm{fMet}}$ ) on the start codon in the P site of the ribosome. In bacteria, initiation is promoted by three initiation factors (IF1, IF2 and IF3) and comprises of several phases (Fig. 5) (Allen and Frank, 2007; Boelens and Gualerzi, 2002; Gualerzi et al., 2001; Laursen et al., 2005; Milon and Rodnina, 2012; Myasnikov et al., 2009; Simonetti et al., 2009).

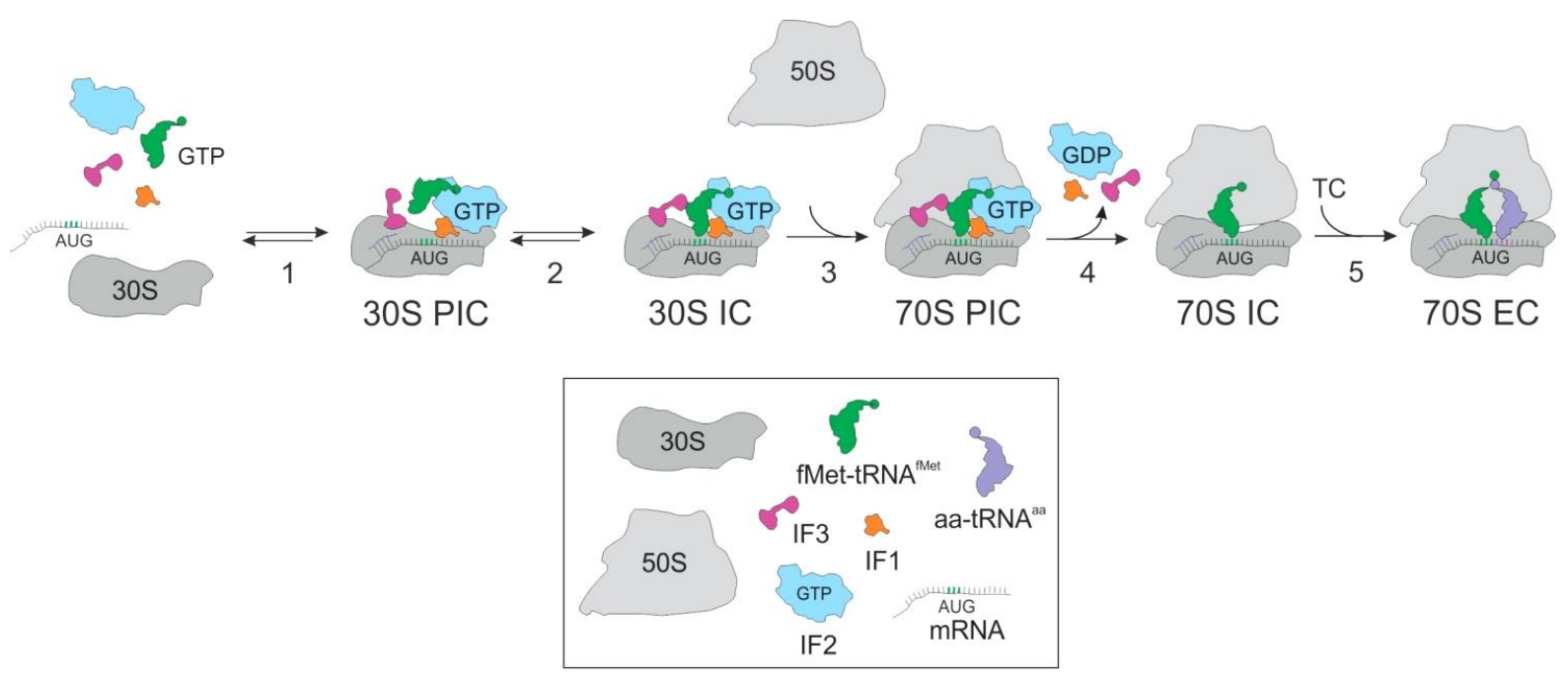

Figure 5. Schematic of translation initiation in bacteria. Step 1: Recruitment of IF1, IF2, IF3, GTP, mRNA and fMet-tRNA $A^{\text {tMet }}$ to the 30 S subunit to form a 30S PIC. Step 2: Conversion of 30 S PIC into 30 S IC upon start codon recognition by fMet-tRNA ${ }^{\text {fMet }}$. Step 3: Association of the $50 \mathrm{~S}$ ribosomal subunit to form the 70S PIC. Step 4: GTP hydrolysis by IF2; dissociation of IF1, IF2 and IF3 giving rise to an elongation competent 70S IC. Step 5: Binding of EF-Tu ternary complex (TC) and peptide bond formation giving rise to a $70 \mathrm{~S}$ elongation complex (EC) (scheme by Prof. M.V. Rodnina).

First, the $30 \mathrm{~S}$ ribosomal subunit binds the IFs, fMet-tRNA ${ }^{\text {fMet }}$ and the mRNA to form a $30 \mathrm{~S}$ PIC. The binding of IFs to the $30 \mathrm{~S}$ subunit is rapid (millisecond range) and follows a kinetically favored order of events with IF3 being the first to bind, followed by IF2 and IF1, respectively (Milon et al., 2012). The factors bind to the 30 S subunit in a cooperative way in a sense that they affect the affinity of each other's binding in the complex. IF1 increases the affinity of IF2 and IF3 towards the 30S subunit (Milon et al., 2012), and is in return stabilised on the $30 \mathrm{~S}$ by their presence (Caserta et al., 2006; Celano et al., 1988; Milon et al., 2012; Stringer et al., 1977; Weiel and Hershey, 1982; Zucker and Hershey, 1986). IF3 allosterically controls the interaction of IF2 with the 30S IC (MacDougall and Gonzalez, 2015; Wang et al., 2015) and together with IF1, stabilizes IF2 on the complex. Vice versa, the binding of IF3 to 
the $30 \mathrm{~S}$ subunit is also stabilized by the presence of IF2 (Milon et al., 2012). The timing of mRNA binding is variable and occurs independently of the IFs bound to the $30 \mathrm{~S}$ subunit (Milon et al., 2012). mRNA recruitment to the stand-by site of the $30 \mathrm{~S}$ subunit, its unfolding and transfer to the initiation site constitute important checkpoints for mRNA selection (Brandt and Gualerzi, 1991; de Smit and van Duin, 2003; La Teana et al., 1995; Marzi et al., 2007; Milon et al., 2012; Studer and Joseph, 2006; Yusupova et al., 2006). Lastly, the C-terminal domain of IF2 recruits fMet-tRNA ${ }^{\text {fMet }}$ to the 30 S complex via specific interactions with its $3^{\text {' }}$ CCA-end (Guenneugues et al., 2000; Milon et al., 2010; Spurio et al., 2000).

Next, the anticodon of fMet-tRNA ${ }^{f M e t}$ recognizes the mRNA start codon in the $P$ site of the $30 S$ subunit and the complex matures into a $30 S$ initiation complex (30S IC) (Milon et al., 2012; Milon and Rodnina, 2012). At this stage, the binding of mRNA, fMet-tRNA ${ }^{\mathrm{fMet}}$, IF1 and IF2 to the 30 S subunit is further strengthened, while the binding of IF3 is destabilised (Milon et al., 2012).

Thereafter, the 50 S subunit binds to the 30 S IC. Synergistic positioning effect of IF2 and fMet-tRNA $^{\text {fMet }}$ on the 30 S IC drives rapid subunit association (Antoun et al., 2006b; GrunbergManago et al., 1975; Simonetti et al., 2008; Wang et al., 2015). One of the ways in which IF1 and IF3 influence the kinetics of 50 S docking is by modulating the orientation and dynamics of IF2-GTP-fMet-tRNA ${ }^{\text {fMet }}$ complex on the 30S IC (Julian et al., 2011; MacDougall and Gonzalez, 2015; Simonetti et al., 2008). In addition, IF3 sterically hinders the formation of inter-subunit bridges (Dallas and Noller, 2001; Julian et al., 2011), thereby slowing down $50 S$ subunit docking. Together, IF1 and IF3 help to discriminate against incorrectly formed $30 \mathrm{~S}$ ICs, such as those programmed with an mRNA containing a non-optimal TIR or lacking IF2 or fMet-tRNA ${ }^{f M e t}$, by inducing a conformation of the $30 S$ IC which impedes $50 S$ subunit joining (Antoun et al., 2006b; Belotserkovsky et al., 2011; Grigoriadou et al., 2007b; Milon et al., 2008; Qin and Fredrick, 2009).

The subsequent maturation of the $70 \mathrm{~S}$ complex is a multistep process (Grigoriadou et al., 2007a; Marshall et al., 2009; Milon et al., 2008; Qin et al., 2009; Tomsic et al., 2000). The current model for 70 S IC formation - based on biochemical, rapid kinetic, and singlemolecule FRET studies - suggests that 50 S subunit joining triggers rapid GTP hydrolysis by IF2, leading to a series of ribosome and IF2 conformational changes and fMet-tRNA ${ }^{\mathrm{fMet}}$ movements (Allen et al., 2005; Grigoriadou et al., 2007a; Huang et al., 2010; Myasnikov et al., 2005; Qin et al., 2009). The fMet-tRNA ${ }^{\text {fMet }}$ is released from the C2-domain of IF2 into the canonical P/P site (Allen et al., 2005; Grigoriadou et al., 2007a; Julian et al., 2011; 
Myasnikov et al., 2005; Simonetti et al., 2008), and the subunits rotate with respect to one another into the classical state, allowing the ribosome to enter an elongation-competent conformation (Marshall et al., 2009).

Finally, IF2 presumably dissociates from the 70S complex (Allen et al., 2005; Antoun et al., 2003; Lockwood et al., 1972; Luchin et al., 1999; Myasnikov et al., 2005), allowing the aminoacyl-tRNA in the ternary complex with EF-Tu and GTP (EF-Tu TC) to bind to the A site and form the first peptide bond. IF3 dissociation from the ribosome also follows subunit joining, as demonstrated by rapid kinetics (Milon et al., 2008), chemical probing (Fabbretti et al., 2007), and single molecule FRET (Elvekrog and Gonzalez, 2013; MacDougall and Gonzalez, 2015). The timing of IF1 release from the ribosome is, however, not yet known. 


\subsection{Role of GTP hydrolysis by IF2}

Attempts at deciphering the significance of IF2 GTPase activity in translation initiation have been made using two main approaches. The first approach is to mutate conserved residues in the $G$ domain implicated in the intrinsic GTPase activity of IF2. The second approach involves the use of non-hydrolysable GTP analogs such as GDPNP, GTPYS or GDPCP.

It has been shown that GTP hydrolysis is not required for the IF2-fMet-tRNA ${ }^{\text {fMet }}$ interaction, 30S IC formation or 50S subunit joining (Anderson et al., 1967; Antoun et al., 2003; Qin et al., 2009; Tsai et al., 2012). After subunit joining and before GTP hydrolysis, the ribosome is in a 'rotated' state where the $30 \mathrm{~S}$ subunit is found in a rotated orientation with respect to the 50S subunit. After GTP hydrolysis, a $5^{\circ}$ inter-subunit rotation occurs, allowing the $70 \mathrm{~S} \mathrm{IC} \mathrm{to}$ enter an elongation-competent 'non-rotated' state (Allen et al., 2005; Marshall et al., 2009). A series of studies yielding contradictory results have made it difficult to reach a consensus regarding the function of GTPase activity of IF2. Evidence obtained using GTPase-deficient mutants of IF2 indicated that GTP hydrolysis is essential for IF2 dissociation from the 70S complex (Fabbretti et al., 2012; Laalami et al., 1994; Luchin et al., 1999). It was also proposed that the energy released by GTP hydrolysis may promote proper positioning of the fMet-tRNA $^{\text {fMet }}$ in the P site (Thach and Thach, 1971). However, on the basis of biochemical and kinetic data it was shown that the GTPase activity of IF2 plays no role in promoting the release of IF2 from the 70S IC (Tomsic et al., 2000) or the positioning of fMet-tRNA ${ }^{\text {fMet }}$ (La Teana et al., 1996; Tomsic et al., 2000).

Cryo-EM reconstructions have provided structural information regarding conformational states of IF2 and fMet-tRNA ${ }^{\text {fMet }}$ on the 70S IC, in the presence of different non-hydrolysable GTP analogs (Allen et al., 2005; Myasnikov et al., 2005). When 70S IC was formed in the presence of GDPNP, all three IFs remained bound to the 70S complex and a stable interaction between the $3^{\prime} \mathrm{CCA}$-end of fMet-tRNA ${ }^{\text {iMet }}$ and IF2 was observed (Allen et al., 2005). On the contrary, in the presence of a different GTP analog, GDPCP, IF2 was present on the 70S complex but no interaction between IF2 and the fMet-tRNA ${ }^{\text {fMet }}$ was detected, leaving the role of GTP hydrolysis in triggering fMet-tRNA ${ }^{\text {fMet }}$ release from IF2 unclear (Myasnikov et al., 2005).

IF2 and EF-Tu occupy overlapping binding sites on the ribosome. Hence, in the absence of IF2 dissociation, the subsequent binding of EF-Tu TC to the A site is expected to be prevented (Antoun et al., 2003; Benne et al., 1973; Tsai et al., 2012). In agreement with this hypothesis, it was reported that the substitution of GTP on IF2 with the non-hydrolysable 
analog, GDPNP, completely inhibited peptide bond formation (Antoun et al., 2003). Contradictory results were obtained in the presence of GDPCP, which lowered the extent of peptide bond formation to approximately half but did not abolish it (Grigoriadou et al., 2007a). Also, on the basis of structural and rapid-kinetic studies, it was suggested IF2 may remain bound to the 70S complex even after GTP hydrolysis (Grigoriadou et al., 2007a; Myasnikov et al., 2005), posing no hindrance to the incoming EF-Tu TC.

Discrepancies in results from the above mentioned studies may arise from (i) the contamination of commercially purchased preparations of GDP or non-hydrolysable GTP analogs with minor quantities of GTP, (ii) the use of different non-hydrolysable GTP analogs which may confer different functional states on IF2 (Simonetti et al., 2009); the GDPNPbound state might represent the GTP form (Allen et al., 2005; Antoun et al., 2003), while the GDPCP-bound state may represent the functional conformation of the factor after GTP hydrolysis but before Pi release (Grigoriadou et al., 2007a; Myasnikov et al., 2005), (iii) the use of initiation components purified from different organisms; G. stearothermophilus (Tomsic et al., 2000), E. coli (Allen et al., 2005; Antoun et al., 2003; Milon et al., 2008; Qin et al., 2009) and T. thermophilus (Myasnikov et al., 2005), and (iv) the use of a heterogeneous translation system, which consists of ribosomal components purified from two different organisms, for example, from G. stearothermophilus and E. coli (Grigoriadou et al., 2007a; Grigoriadou et al., 2007b) or a homogeneous system (Allen et al., 2005; Antoun et al., 2003; Milon et al., 2008). 


\subsection{Scope of the thesis}

The timing of several reactions which occur during the transition from the 30S IC into the translating 70 S ribosome, as well as the interplay between the IFs during this process, are to a large extent disputed or unknown. Kinetic data from different groups have yielded contradictory results regarding the sequence of events and the importance of IF2-dependent GTP hydrolysis In this thesis, the timing and control of several reactions which occur during 70S IC formation were investigated by rapid kinetic techniques using a toolbox of fluorescence-labeled translation components. An in vitro system of translation initiation reconstituted with components purified from E. coli was utilized. The kinetics of IF1 and IF3 dissociation from the 70S complex, the release of the fMet-tRNA ${ }^{\text {fMet }}$ CCA-end and GDP from IF2, as well as the participation of IF2 in the second round of translation initiation were followed with the help of novel observables. Previously studied reactions such as subunit joining, Pi release from IF2, and peptide bond formation (Grigoriadou et al., 2007a; Milon et al., 2008; Tomsic et al., 2000; Wishnia et al., 1975) were also monitored to formulate a kinetic model inclusive of all events which occur during the maturation of $30 \mathrm{~S} \mathrm{IC} \mathrm{into} \mathrm{an}$ elongation-competent 70S IC. In addition, the requirement for IF1 and IF3 for every reaction, as well as the dependency on IF2-dependent GTP hydrolysis was examined. The effect of phosphate analogs on subunit joining and the IF2-tRNA ${ }^{\text {fMet }}$ interaction was also checked. The results provide a comprehensive kinetic scheme for 70S IC formation, give insights into the interplay between the IFs and underscore the role of GTP hydrolysis by IF2 during translation initiation. 


\section{RESULTS}

\subsection{Experimental setup}

The transition of the 30S IC into an elongation-ready 70S IC is a multi-step process consisting of several binding, chemical hydrolysis and dissociation events which occur in the $\mathrm{ms}$ - s time scale. Rapid kinetic approaches can be used to monitor these events in real-time before the entire process reaches completion. Hence, pre-steady state kinetics can help dissect the biochemical pathway and identify reaction intermediates. We monitored 70S IC formation by rapidly mixing $30 \mathrm{~S} \mathrm{IC}$, containing a fluorescent or radioactive reporter, with $50 \mathrm{~S}$ subunits in the stopped- or quench-flow apparatus. The progression of each reaction was followed by monitoring the changes in fluorescence or FRET (Förster Resonance Energy Transfer) with time. In this way, the timing of several processes which occur during 70 S IC formation, such as (i) $50 \mathrm{~S}$ subunit joining, (ii) changes in IF1 environment and dissociation of the factor from the ribosome, (iii) IF2 dissociation from the ribosome, (iv) IF3 dissociation from the ribosome, (v) GTPase activation of IF2, (vi) Pi release from IF2, (vii) fMet-tRNA ${ }^{\text {fMet }}$ release from IF2, (viii) GDP release from IF2, (ix) binding of IF2 to 30 S complexes during the next round of initiation, and $(x)$ the first peptide bond formation, indicative of transition into the elongation phase of translation, was determined.

30 S IC was formed using translation initiation components purified from E. coli. 30 S subunits were incubated with a 3-fold molar excess of IF1, IF2 and IF3 (or a 2-fold molar excess of fluorescent IFs) and a 5 -fold molar excess of mRNA and f[ $\left.{ }^{3} \mathrm{H}\right] \mathrm{Met}_{\text {-tRNA }}{ }^{\mathrm{fMet}}$ (or 3-fold molar

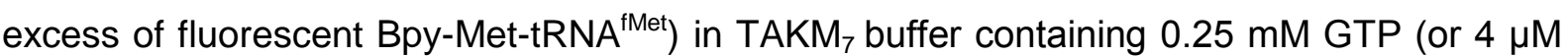
fluorescent Bpy-GTP/ $10 \mu \mathrm{M}$ mant-GTP) for $30 \mathrm{~min}$ at $37^{\circ} \mathrm{C}$. As a model mRNA, m022 (La Teana et al., 1993) was chosen, because the 30S IC formed with this mRNA rapidly associates with the 50S subunit (Grigoriadou et al., 2007a; Milon et al., 2008), which is essential to resolve the individual rates of the subsequent reactions. Because fluorescence labeling of a protein or RNA can adversely affect its function, the activity of each labeled component in promoting subunit association was compared with its unlabeled counterpart.

To help identify the biochemical reaction depicted by each reporter, the binding and dissociation of the observable in relation to the $30 \mathrm{~S}$ complex was studied. To solve the kinetic mechanism of 70 S IC formation, each reaction was monitored under pseudo-first order conditions, where the 30S IC was mixed with a 3-20 fold molar excess of 50 S subunits. Time courses were obtained at increasing $50 \mathrm{~S}$ subunit concentrations and fitted with an exponential function to derive the apparent rate $\left(k_{\text {app }}\right)$ constant of the reaction. Bimolecular 
events such as binding reactions show a linear dependence of apparent rates on increasing 50S concentration, whereas subsequent monomolecular rearrangements display a hyperbolic dependence. However, if the reaction was preceded by a delay phase, exponential fitting could not be utilized. To assign the order and timing of the respective steps, the time courses were initially evaluated on the basis of their apparent rate constants and the length of the delay phase preceding the respective reaction. Subsequently, the elemental rate constant of each reaction was determined from the global fitting of time courses for all observables with a 10-step kinetic model, to provide a comprehensive solution for the processes involved in 70S IC formation (section 5.8).

The requirement for GTP hydrolysis by IF2 in promoting different reactions was examined by substituting GTP with a non- (or very slowly) hydrolysable GTP analog, GTPyS. Recent studies on elF5B, the eukaryotic homolog of IF2, have deemed GTPYS as a suitable GTP analog due to its ability to correctly coordinate a monovalent cation in the active site of translational GTPases (Kuhle and Ficner, 2014b). Similar conclusions were reached in case of SelB (Paleskava et al., 2012) where GTPYS was shown to be an authentic GTP analog. The reactions were monitored at a fixed concentration $(1 \mu \mathrm{M})$ of $50 \mathrm{~S}$ subunits.

To study the individual roles of IF1 and IF3 in actively promoting each process, the reactions were monitored in the presence and absence of either factor at a fixed concentration (1 $\mu \mathrm{M})$ of $50 \mathrm{~S}$ subunits. No experiments were done in the absence of IF2 because its omission results in impaired fMet-tRNA ${ }^{\text {fMet }}$ recruitment and $50 \mathrm{~S}$ subunit association (Grunberg-Manago et al., 1975). 


\subsection{Screening the activity of translation initiation components}

50 S binding to 30 S IC can be followed by monitoring the increase in light scattering (LS) which occurs when the subunits associate with each other to form a 70S complex. The rate and amplitude change of LS is dependent on several parameters, such as the composition of the mRNA TIR, the integrity of ribosomal subunits, the identity of the nucleotide bound to IF2 and the presence and activity of different IFs and fMet-tRNA ${ }^{\text {fMet }}$. The amplitude change of LS reflects the amount of 30S IC in the reaction, whereas the rate indicates whether these complexes are in the correct conformation for entering the translation pathway.

The results shown below provide an overview of the importance of different initiation components in promoting subunit joining (Antoun et al., 2004; Grunberg-Manago et al., 1975) and emphasize the effectiveness of monitoring changes in LS to check the activity of purified preparations of ribosomes, IFs, RNA and nucleotides. 30S IC was prepared in the presence of all components, or in the absence of individual components, to study their role in promoting IC formation and subunit joining (Fig. 6A). Upon rapidly mixing $30 \mathrm{~S} \mathrm{IC}(0.05 \mu \mathrm{M})$ with 50 s subunits $(1 \mu \mathrm{M})$ in the stopped-flow apparatus, a biphasic increase in LS was observed (Grigoriadou et al., 2007a; Milon et al., 2008) (Fig. 6B). The predominant rapid phase ( $>75 \%$ of the total amplitude change) is indicative of $50 \mathrm{~S}$ joining to the majority of $30 \mathrm{~S}$ ICs which are present in a 'productive' conformation, whereas the minor phase probably represents a small portion of less active (McGinnis et al., 2015) or compositionally heterogeneous complexes. In the presence of all components, the apparent rate of the predominant phase was $10 \mathrm{~s}^{-1}$. No signal change was present in the absence of $50 \mathrm{~S}$ subunits. When $30 \mathrm{~S} \mathrm{IC}$ was formed in the absence of IF1 or IF3, the apparent rate of subunit joining was 2 and 5 -fold faster, respectively (Fig. 6C). The absence of IF2, GTP or fMettRNA $^{\text {fMet }}$ slowed subunit joining by more than 500 -fold $\left(0.007-0.02 \mathrm{~s}^{-1}\right)$ (Fig. 6D-F), in agreement with previous reports that the sub-complex between IF2, GTP and fMet-tRNA ${ }^{\text {iMet }}$ on the 30S IC drives rapid subunit association (Antoun et al., 2003; Antoun et al., 2006b). 
(A)

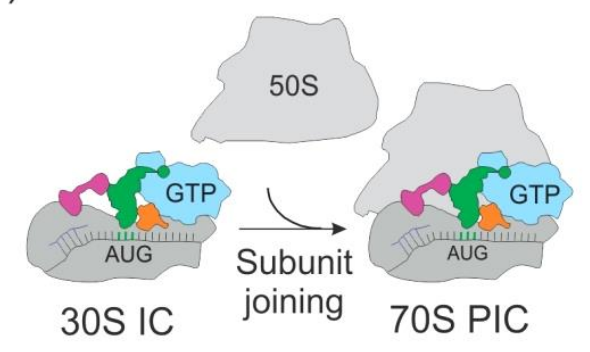

(C)

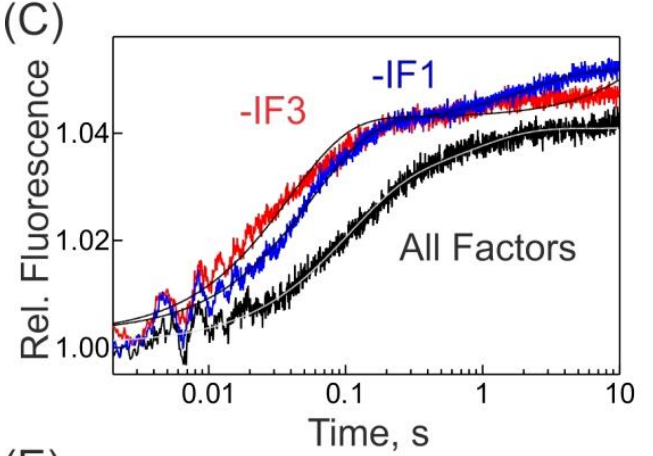

(E)

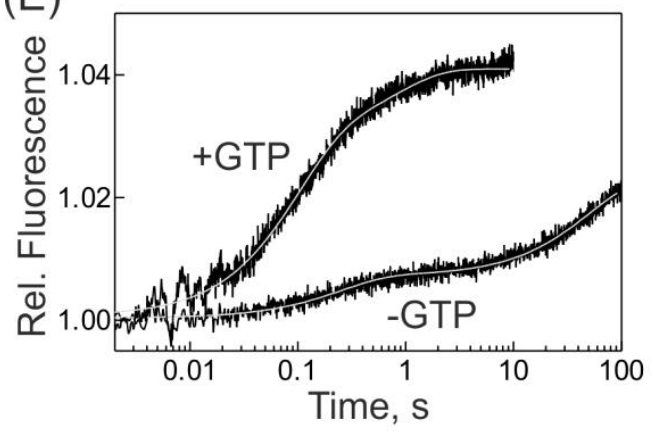

(B)
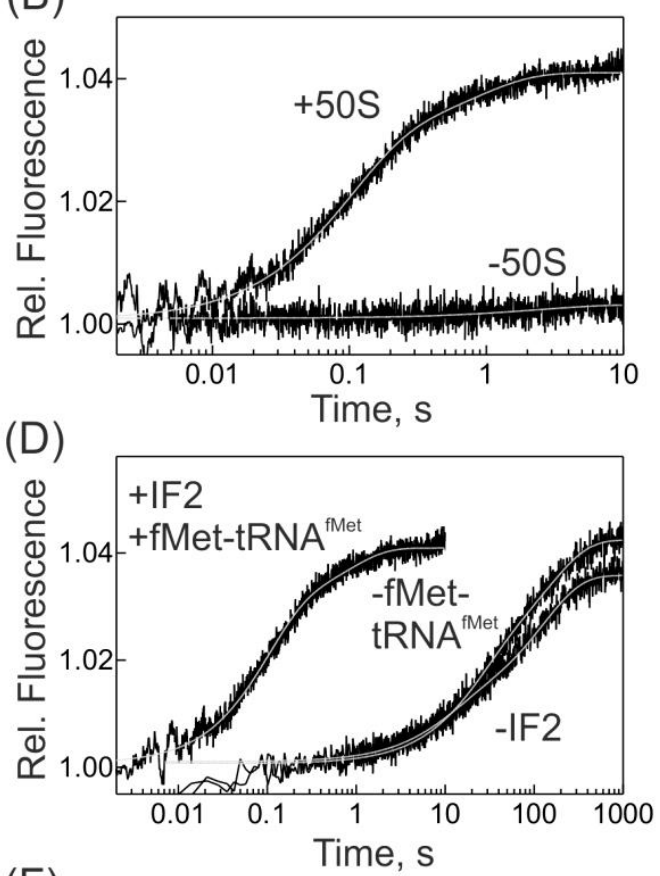

$(\mathrm{F})$

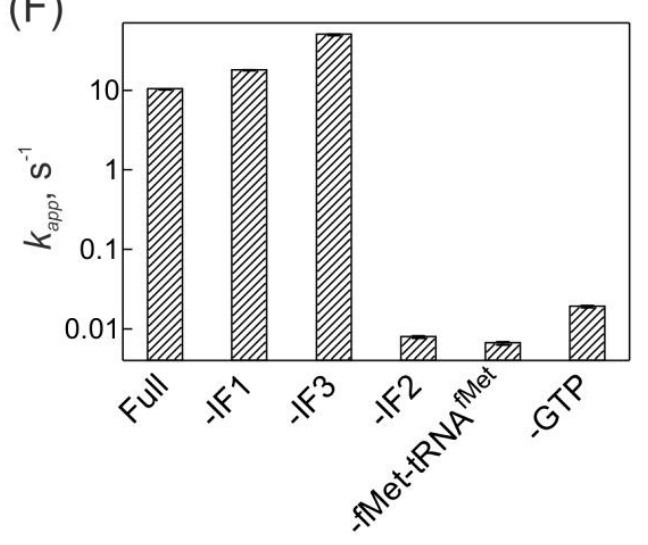

Figure 6. Role of translation initiation components in promoting subunit association. 30 S IC $(0.05 \mu \mathrm{M})$ was rapidly mixed with 50 S subunits $(0.05 \mu \mathrm{M})$ in a stopped-flow apparatus and the change in LS upon subunit association was monitored. (A) Schematic of 50 S joining to 30 S IC forming a 70S PIC. (B) Time courses of LS upon mixing 30S IC with 50 S subunits or buffer (-50S). (C) Time courses of subunit joining to $30 \mathrm{~S} \mathrm{IC} \mathrm{in} \mathrm{the} \mathrm{presence} \mathrm{of} \mathrm{all} \mathrm{factors}$ and absence of IF1 or IF3. (D) Time courses of subunit joining to $30 \mathrm{~S} \mathrm{IC} \mathrm{in} \mathrm{the} \mathrm{presence} \mathrm{and}$ absence of IF2 or fMet-tRNA ${ }^{\text {fMet }}$. (E) Time courses of subunit joining to $30 \mathrm{~S} \mathrm{IC} \mathrm{in} \mathrm{the}$ presence and absence of GTP. (F) Apparent rate of the predominant phase derived from double-exponential fitting of time courses in $(B)-(E)$; error bars represent standard error of the fit. 
The kinetics of $50 \mathrm{~S}$ joining to the 30 S IC is a very sensitive indicator for checking the functionality of the translation initiation machinery and was hence used to test the activity of fluorescence-labeled initiation components. If the activity of the 30S, 50S, IF2, GTP or fMettRNA $^{\mathrm{fMet}}$ preparations is decreased, e.g. by introducing mutations, fluorescence reporters or inactivation during purification, the rate and/or extent of LS is expected to be lower. On the other hand, preparations of IF1 or IF3 with low activity would increase the rate and/or extent of LS (Antoun et al., 2003; Antoun et al., 2006b; Antoun et al., 2004; Grunberg-Manago et al., 1975; Milon et al., 2008). Slow subunit docking would rate-limit subsequent reactions involved in 70S IC formation; hence, only reporters competent in promoting rapid subunit association were utilized to study these events.

\subsubsection{Fluorescent ribosomal subunits}

$30 S$ subunits lacking the ribosomal protein S13 (30S $\Delta$ S13) or 50 S subunits lacking L33 (50S $\Delta \mathrm{L} 33$ ) were purified from $E$. coli strain $\mathrm{K} 12$, and subsequently reconstituted with fluorescence-labeled S13 and L33 proteins, respectively. The activity of ribosomal subunits purified from E. coli strain $\mathrm{K} 12$ was compared with wild-type subunits purified from $E$. coli strain MRE600 (the standard strain used in our laboratory) (Fig. 7A). Replacement of 30S subunits from MRE600 with those from K12 did not significantly affect the rate of subunit joining $\left(5 \mathrm{~s}^{-1}\right.$ at $30 \mathrm{~S} I \mathrm{C}(0.05 \mu \mathrm{M})$ and 50 S subunit $(0.25 \mu \mathrm{M})$ concentration), but a $\sim 20 \%$ lower amplitude was observed, indicating that a small fraction of the ribosomal population was not active in initiation. The fluorescence labeling of the $30 \mathrm{~S}$ subunits with thiol-reactive dyes such as Alexa488 $\left(30 \mathrm{~S}_{\mathrm{S} 13}(\mathrm{Al} \times 488)\right)$ or Atto540Q $\left(30 \mathrm{~S}_{\mathrm{S} 13}(\mathrm{Atto540Q})\right.$ ), also did not affect the kinetics of subunit joining (Fig. 7B). Thus, despite the slightly lower extent of subunit joining, the fluorescence-labeled $30 \mathrm{~S}$ subunits could be used to study reaction kinetics during $70 S$ IC formation. When $50 S$ subunits from E. coli strain $\mathrm{K} 12$ were used, the extent of subunit joining was similar to that observed in the presence wild-type $50 S$ subunits from $E$. coli strain MRE600 (Fig. 7A). Similar results were obtained when fluorescence-labeled 50S subunits $\left(50 \mathrm{~S}_{\mathrm{L33}}(\mathrm{Al} \times 488)\right.$ and $50 \mathrm{~S}_{\mathrm{L33}}($ Atto540Q)) were tested (Fig. 7C). However, because the rate of subunit joining was $\sim 10$-fold slower $\left(0.5 \mathrm{~s}^{-1}\right)$ (Fig. 7D), the fluorescent $50 \mathrm{~S}$ subunits could not be utilized to study the kinetics of reactions which take place after subunit association. 
(A)

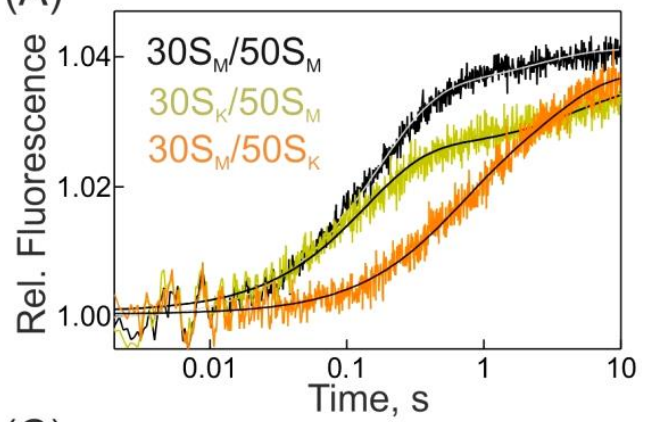

(C)

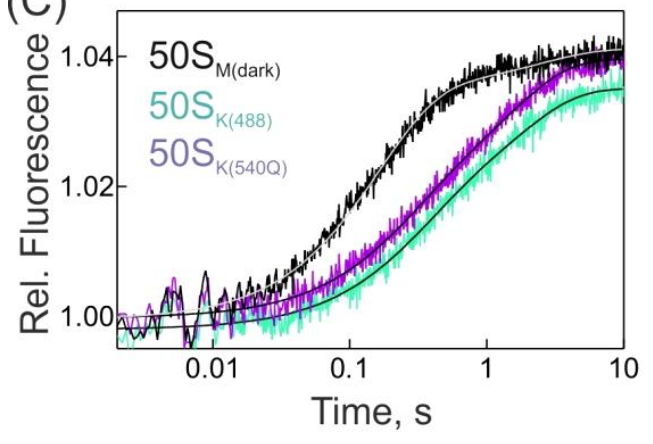

(B)

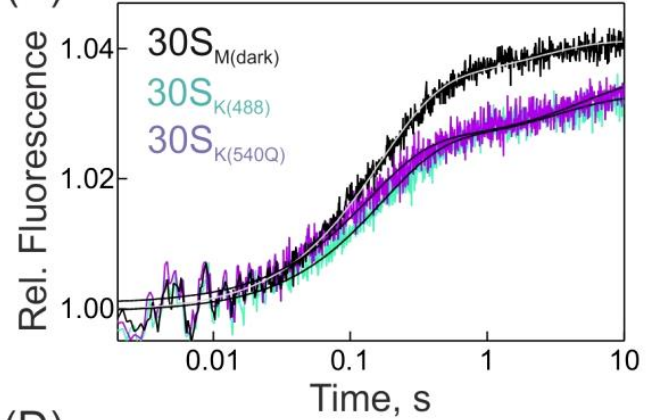

(D)

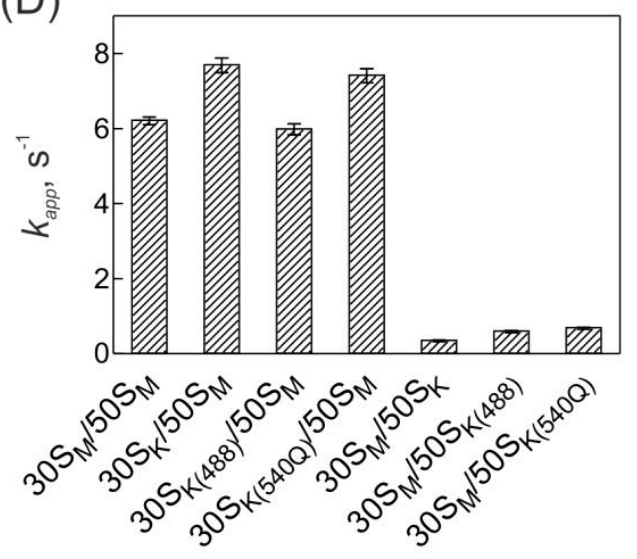

Figure 7. Effect of fluorescence-labeled ribosomal subunits on subunit association. 30 S IC $(0.05 \mu \mathrm{M})$ was rapidly mixed with 50 S subunits $(0.25 \mu \mathrm{M})$ in a stopped-flow apparatus and the change in LS upon subunit association was monitored. (A) Time courses of subunit association using subunits purified from E. coli strain MRE600 (M) or strain K12 (K). (B) Time courses of subunit association using non-fluorescent (MRE600) or fluorescence-labeled (K12; Alexa488 or Atto540Q) 30S subunits. (C) Time courses of subunit association using non-fluorescent (MRE600) or fluorescence-labeled (K12; Alexa488 or Atto540Q) 50S subunits. (D) Apparent rate of the predominant phase derived from double-exponential fitting of time courses in (A)-(C); error bars represent standard error of the fit.

\subsubsection{Fluorescent initiation factors}

Cysteine residues were introduced at position 4 in IF1, position 757 in IF2 and position 166 in IF3 and labeled with thiol-reactive fluorescent (Alexa488 or Alexa555) or quencher (Atto540Q) dyes. All labeled IFs were competent in promoting rapid subunit association (Fig. 8), and thus could be utilized to monitor timings of reactions which follow subunit joining. 
(A)

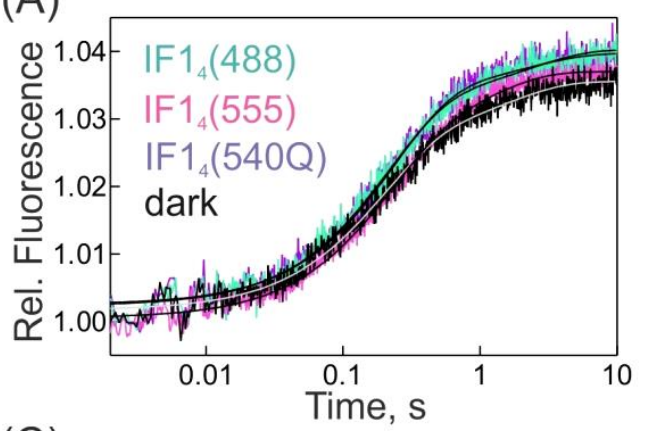

(C)

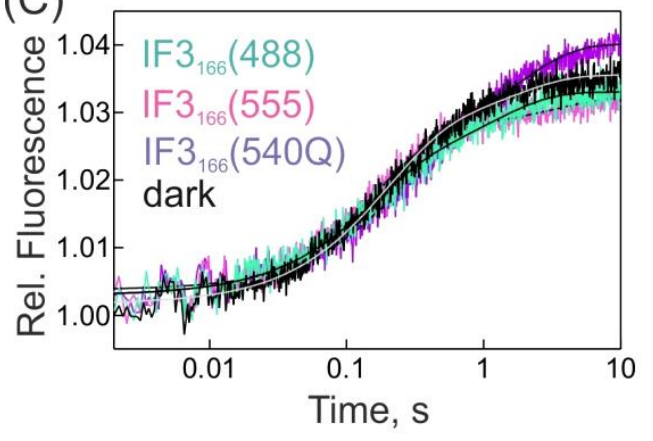

(B)

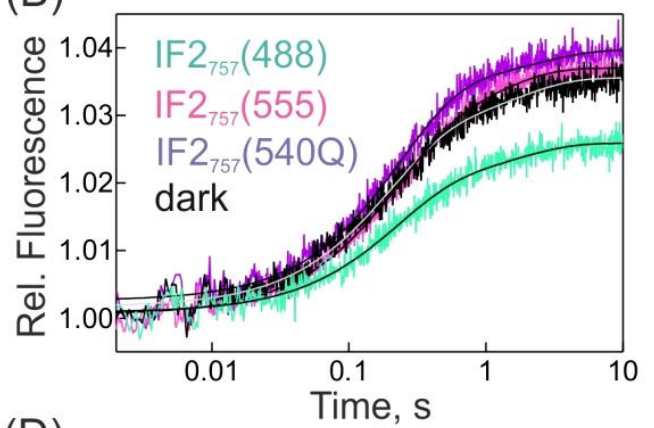

(D)

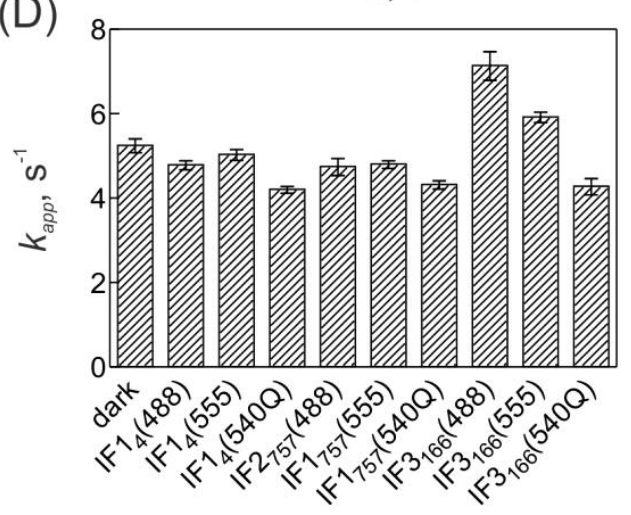

Figure 8. Effect of fluorescence-labeled IFs on subunit association. 30S IC $(0.05 \mu \mathrm{M})$, formed in the presence of non-fluorescent IFs (dark) or fluorescent IFs, was rapidly mixed with 50 S subunits $(0.25 \mu \mathrm{M})$ in a stopped-flow apparatus and the change in LS upon subunit association was monitored. (A) Time courses of subunit association in the presence of indicated IF1 variants. (B) Time courses of subunit association in the presence of indicated IF2 variants. (C) Time courses of subunit association in the presence of indicated IF3 variants. (D) Apparent rate of the predominant phase derived from double-exponential fitting of time courses in $(A)-(C)$; error bars represent standard error of the fit.

\subsubsection{Fluorescent tRNA ${ }^{\mathrm{fMet}}$ and GTP}

Fluorescent derivatives of fMet-tRNA ${ }^{\mathrm{fMet}}$ and GTP were used to monitor the interaction of these components with IF2 on the 70S IC. The tRNA ${ }^{\text {fMet }}$ was labeled at either the $\alpha$-amino group of methionine with the fluorophore Bodipy-FL (Holtkamp et al., 2014) or with fluorescein at the modified thio-U nucleotide found naturally in tRNA ${ }^{\text {fMet }}$ (Milon et al., 2007). Fluorescent analogues of GTP, such as mant-GTP and Bpy-GTP where the fluorophore is attached to the sugar backbone, were purchased from commercial sources. The use of BpyMet-tRNA ${ }^{\text {fMet }}$ (Fig. 9A) decreased the extent of LS by almost 50\%, while allowing the reaction to occur with the rate obtained using non-fluorescent tRNA $A^{\mathrm{fMet}}\left(5 \mathrm{~s}^{-1}\right)$. 
(A)

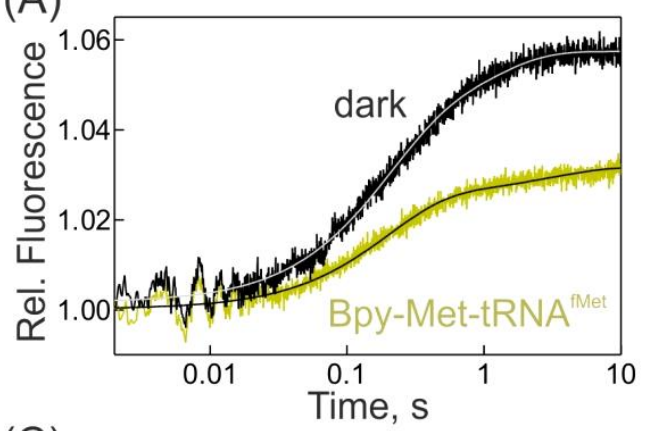

(C)

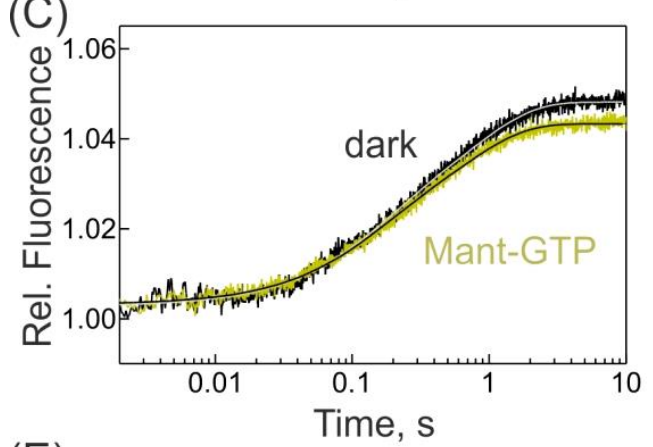

(E)

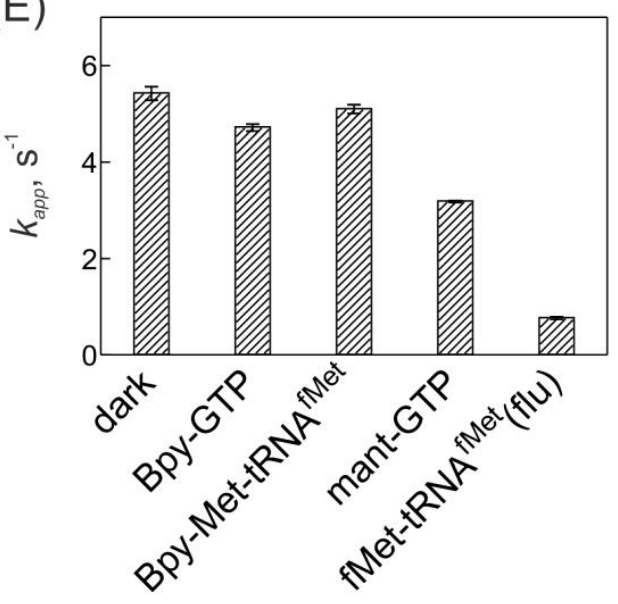

(B)

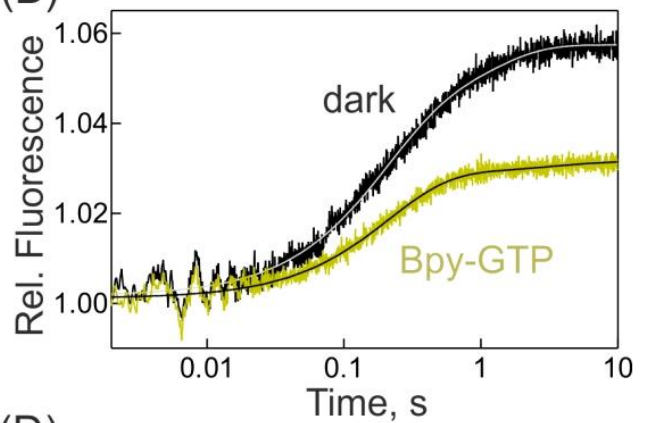

(D)

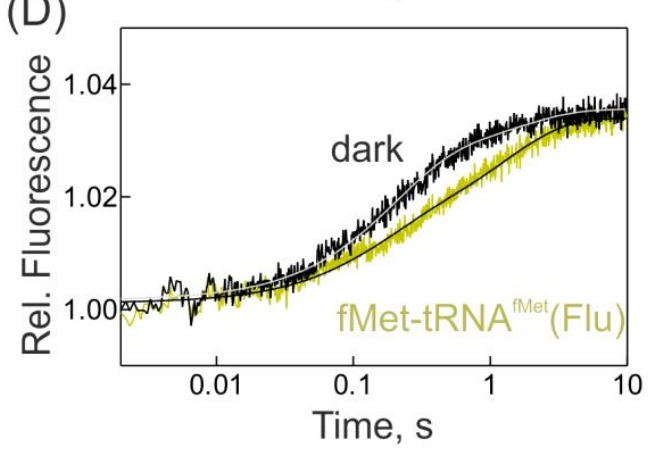

Figure 9. Effect of fluorescence-labeled tRNA ${ }^{\text {fMet }}$ and GTP on subunit association. $30 \mathrm{~S}$ IC $(0.05 \mu \mathrm{M})$, formed in the presence of non-fluorescent (dark) or fluorescent components, was rapidly mixed with $50 \mathrm{~S}$ subunits $(0.25 \mu \mathrm{M})$ in a stopped-flow apparatus and the change in LS upon subunit association was monitored. (A) Time courses of subunit association in the presence of Bpy-Met-tRNA ${ }^{\text {fMet }}$. (B) Time courses of subunit association in the presence of Bpy-GTP. (C) Time courses of subunit association in the presence of mant-GTP. (D) Time courses of subunit association in the presence of fMet-tRNA ${ }^{\text {fMet }}$ (Fluorescein). (E) Apparent rate of the predominant phase derived from double-exponential fitting of time courses in (A)(D); error bars represent standard error of the fit.

To minimize the fluorescence background, 30S IC was formed in the presence of Bpy-GTP $(2 \mu \mathrm{M})$. The affinity of GTP to IF2 increases 20 -fold $\left(K_{d}=40 \mu \mathrm{M}\right.$ to $\left.2 \mu \mathrm{M}\right)$ in the presence of the 30 S subunit and fMet-tRNA ${ }^{\text {fMet }}$ (Antoun et al., 2003; Fabbretti et al., 2012). Assuming that 
the affinity of Bpy-GTP is in the same range as the natural substrate (as indicated by the fact that subunits joining occurs as the same rate (Fig. 9B)), this concentration would allow 50\% of 30S-bound IF2 to bind Bpy-GTP, which is consistent with the $50 \%$ decrease in the extent of subunit joining observed with these complexes. Replacement of GTP with mant-GTP slightly decreased the rate of subunit joining; however, the subunit joining was also slower in the presence of non-fluorescent GTP, indicating that the activity of at least one of the unlabeled initiation components was not optimal in that experiment (Fig. 9C). The use of fluorescein-labeled fMet-tRNA ${ }^{\mathrm{fMet}}$ lowered the rate of subunit joining by 5 -fold $\left(1 \mathrm{~s}^{-1}\right)$ (Fig. 9D). Hence, only the components which did not affect the rates of subunit association i.e, BpyGTP, mant-GTP and Bpy-Met-tRNA ${ }^{\text {fMet }}$ (Fig. 9E) were used to study the timings of tRNA ${ }^{\text {fMet }}$ and GTP-related reactions after subunit joining.

\subsubsection{Dual-labeled 30 S IC}

The interaction between two components can be studied by fluorescence-labeling both and monitoring the changes in FRET between them. If both dyes are fluorescent, the donor and acceptor fluorescence can be monitored. On the other hand, if the donor dye is fluorescent and the acceptor dye is non-fluorescent, only the donor fluorescence is monitored. In the latter case, close proximity between the two components results in a low fluorescence state, which in the following text will be referred to as 'fluorescence quenching'. When the components move apart from each other, an increase in fluorescence (or 'de-quenching') is recorded. The dual-labeling may adversely affect initiation by perturbing interaction between the components on the ribosome. Therefore, the kinetics of subunit joining was tested in the presence of both fluorescence-labeled components.

When 30S IC formed using $30 \mathrm{~S}_{\mathrm{S} 13}(\mathrm{Alx} 488)$ subunit and $\mathrm{IF}_{4}(\mathrm{Atto540Q})$ was tested for subunit association, there was no effect on the kinetics or extent of subunit joining (Fig. 10A). When $30 \mathrm{~S} \mathrm{IC} \mathrm{was} \mathrm{formed} \mathrm{with} 30 S_{S_{13}}\left(\right.$ Alx488) subunits and IF3 ${ }_{166}$ (Atto540Q), a biphasic increase in LS was observed with the slow phase $\left(0.05 \mathrm{~s}^{-1}\right)$ accounting for $\sim 40 \%$ of the amplitude change (Fig. 10B). In the presence of IF2 ${ }_{757}$ (Atto540Q) and IF3 ${ }_{166}(A I x 488)$, the rate of subunit joining was slightly slower than that obtained using the non-fluorescent factors (Fig. 10C-D). Hence, the first FRET pair could be used to study the timing of initiation reactions, whereas the latter two FRET pairs could only be utilized to monitor IF3 movements on the 305 subunit in a qualitative manner, i.e towards or away from the respective initiation component. 

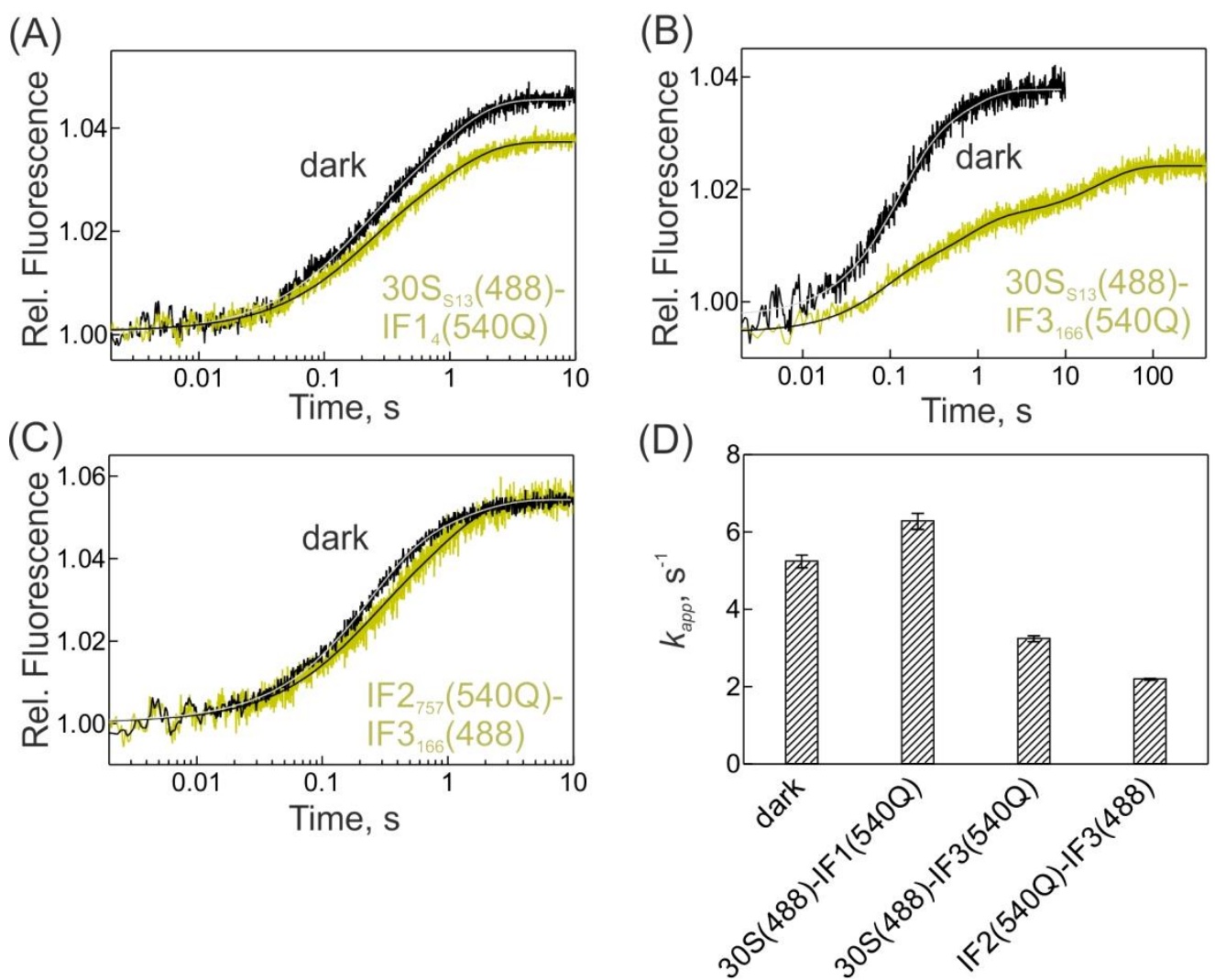

Figure 10. Effect of dual-labeled 30S IC on subunit association. 30S IC $(0.05 \mu \mathrm{M})$ formed in the presence of non-fluorescent (dark) or fluorescent components was rapidly mixed with 50 subunits $(0.25 \mu \mathrm{M})$ in a stopped-flow apparatus and the change in LS upon subunit association was monitored. (A) Time courses of subunit association in the presence of $30 \mathrm{~S}_{133}\left(\mathrm{Alx} 488\right.$ ) and IF1 ${ }_{4}$ (Atto540Q). (B) Time courses of subunit association in the presence of $30 \mathrm{~S}_{\mathrm{S} 13}(\mathrm{Al} \times 488)$ and $\mathrm{IF} 3_{166}$ (Atto540Q). (C) Time courses of subunit association in the presence of IF2 ${ }_{757}$ (Atto540Q) and IF3 ${ }_{166}($ Alx488). (D) Apparent rate of the predominant phase derived from double-exponential fitting of time courses in $(A)-(C)$; error bars represent standard error of the fit. 


\subsection{Dynamics of subunit association}

\subsubsection{Kinetics of subunit joining}

When 30S IC was mixed with increasing concentrations of $50 \mathrm{~S}$ subunits to form a $70 \mathrm{~S}$ complex (Fig. 11A), the apparent rate constant of the first, dominant phase of LS was found to increase linearly with $50 \mathrm{~S}$ concentration, indicative of a bimolecular binding reaction (Fig. 11B).
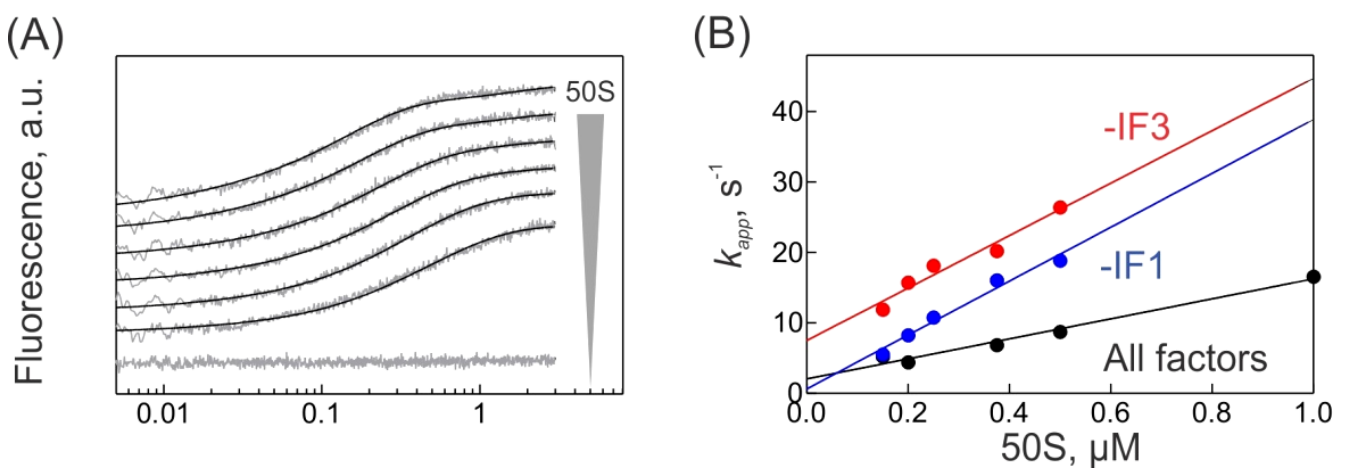

Figure 11. 50S subunit association with the 30S IC. 30 S IC $(0.05 \mu \mathrm{M})$ was rapidly mixed with $50 \mathrm{~S}$ subunits in a stopped-flow apparatus and the change in LS upon subunit association was monitored. (A) Time courses of LS upon rapidly mixing $30 \mathrm{~S}$ IC with increasing concentrations of $50 \mathrm{~S}$ subunits $(0.15-1 \mu \mathrm{M})$. Control measurements were performed in the absence of $50 \mathrm{~S}$ subunits. (B) Dependence of apparent rate $\left(\mathrm{k}_{\text {app }}\right)$ values of the predominant phase of LS on 50S subunit concentration. The reactions were monitored in the presence of all factors and in the absence of IF1 or IF3 (latter experiment performed by Dr. C. Maracci). $k_{\text {app }}$ values were derived from double-exponential fitting of time courses. Error bars (smaller than symbol size) represent standard error of the fit.

In the presence of all factors, the apparent rate constant of subunit joining to 30 S IC was 14 $\mu \mathrm{M}^{-1} \mathrm{~s}^{-1}$ (Table 1; Milon et al., 2008). In the absence of IF1 or IF3, the apparent rate constant of subunit joining was increased to $38 \mu^{-1} \mathrm{~s}^{-1}$ (Fig. 11B; Table 1). This affect is far more pronounced in the presence of mRNAs containing a non-optimal TIR, where IF1 and IF3 impede subunit joining significantly (Grigoriadou et al., 2007b; Milon et al., 2008) and the absence either factor can accelerate subunit association by up to 100 -fold. 
Table 1. Summary of apparent rate constants of reactions during 70S IC formation.

\begin{tabular}{|c|c|c|c|c|c|}
\hline Kinetic step & Observable & $\begin{array}{l}\text { All IFs } \\
\text { GTP }\end{array}$ & $\begin{array}{l}\text {-IF1 } \\
\text { GTP }\end{array}$ & $\begin{array}{l}\text {-IF3 } \\
\text { GTP }\end{array}$ & $\begin{array}{l}\text { All IFs } \\
\text { GTPYS }\end{array}$ \\
\hline $\begin{array}{l}\text { Subunit joining, } \\
k_{\text {app on on }}\left(\mu \mathrm{M}^{-1} \mathrm{~s}^{-1}\right)\end{array}$ & LS & $14 \pm 1$ & $38 \pm 4$ & $37 \pm 4$ & n.d. \\
\hline GTPase activation $\left(\mathrm{s}^{-1}\right)$ & Mant-GTP & $9.1 \pm 0.5$ & $14 \pm 1$ & $27 \pm 1$ & $7.4 \pm 0.5$ \\
\hline $\begin{array}{l}\text { Change of IF1 } \\
\text { environment }\left(\mathrm{s}^{-1}\right)\end{array}$ & $\mathrm{IF} 1_{4}(\mathrm{Al} \times 555)$ & $4.7 \pm 0.5$ & n.o. & $9.7 \pm 0.5$ & $3.7 \pm 0.5$ \\
\hline IF1 dissociation $\left(\mathrm{s}^{-1}\right)$ & $\begin{array}{c}\mathrm{IF}_{4}\left(\mathrm{Atto}_{\mathrm{A}} \mathrm{OQ}\right)- \\
30 \mathrm{~S}_{\mathrm{S13}}(\mathrm{Al} \times 488)\end{array}$ & $1.9 \pm 0.1$ & n.o. & $2.1 \pm 0.1$ & n.o. \\
\hline IF2 dissociation $\left(\mathrm{s}^{-1}\right)$ & Mant-GTP & $2.5 \pm 0.1$ & $2.8 \pm 0.1$ & $2.3 \pm 0.1$ & n.o. \\
\hline IF3 dissociation $\left(\mathrm{s}^{-1}\right)$ & $\begin{array}{l}\mathrm{IF3}_{166}(\text { Alx488) - } \\
\mathrm{IF}_{757} \text { (Atto540Q) }\end{array}$ & $6 \pm 0.5$ & $37 \pm 0.5$ & n.o. & $4.4 \pm 0.5$ \\
\hline
\end{tabular}

All rates and standard errors are derived from exponential fitting of time courses; n.d. - not determined; n.o. - not observed

\subsubsection{Effect of different GTP analogs on subunit joining}

$30 \mathrm{SIC}$, formed in the presence of different pre- and post-hydrolysis state GTP analogs, was rapidly mixed with $50 S$ subunits in a stopped-flow apparatus and the time courses of LS were monitored. In its GTP bound state, the sub-complex formed between IF2 and fMet-tRNA ${ }^{\text {iMet }}$ on the 30S IC promoted rapid subunit association $\left(5 \mathrm{~s}^{-1}\right.$ at $50 \mathrm{~S}$ subunit concentration $(0.25$ $\mu M)$ ) (Fig. 12A). When GTP was replaced with GDP, the conformational dynamics of IF2 did not support $50 \mathrm{~S}$ joining (Antoun et al., 2003). It should be noted that two phases were observed when commercially bought GDP was used for 30 S IC formation. The first phase, which took place with a rate of $5 \mathrm{~s}^{-1}$ and occupied $25 \%$ of the total amplitude change is most likely representative of GTP contamination in the GDP preparation. Because the affinity of GTP to the 30S-bound IF2 is higher than that of GDP, even trace amounts of GTP contamination can result in artifacts which can lead to misinterpretation of data. Hence, GDP was purified using anion-exchange chromatography (Panico et al., 1990) for use in further experiments. In the presence of purified GDP, the first phase almost disappeared and only the slow phase $\left(0.02 \mathrm{~s}^{-1}\right)$ was observed. 

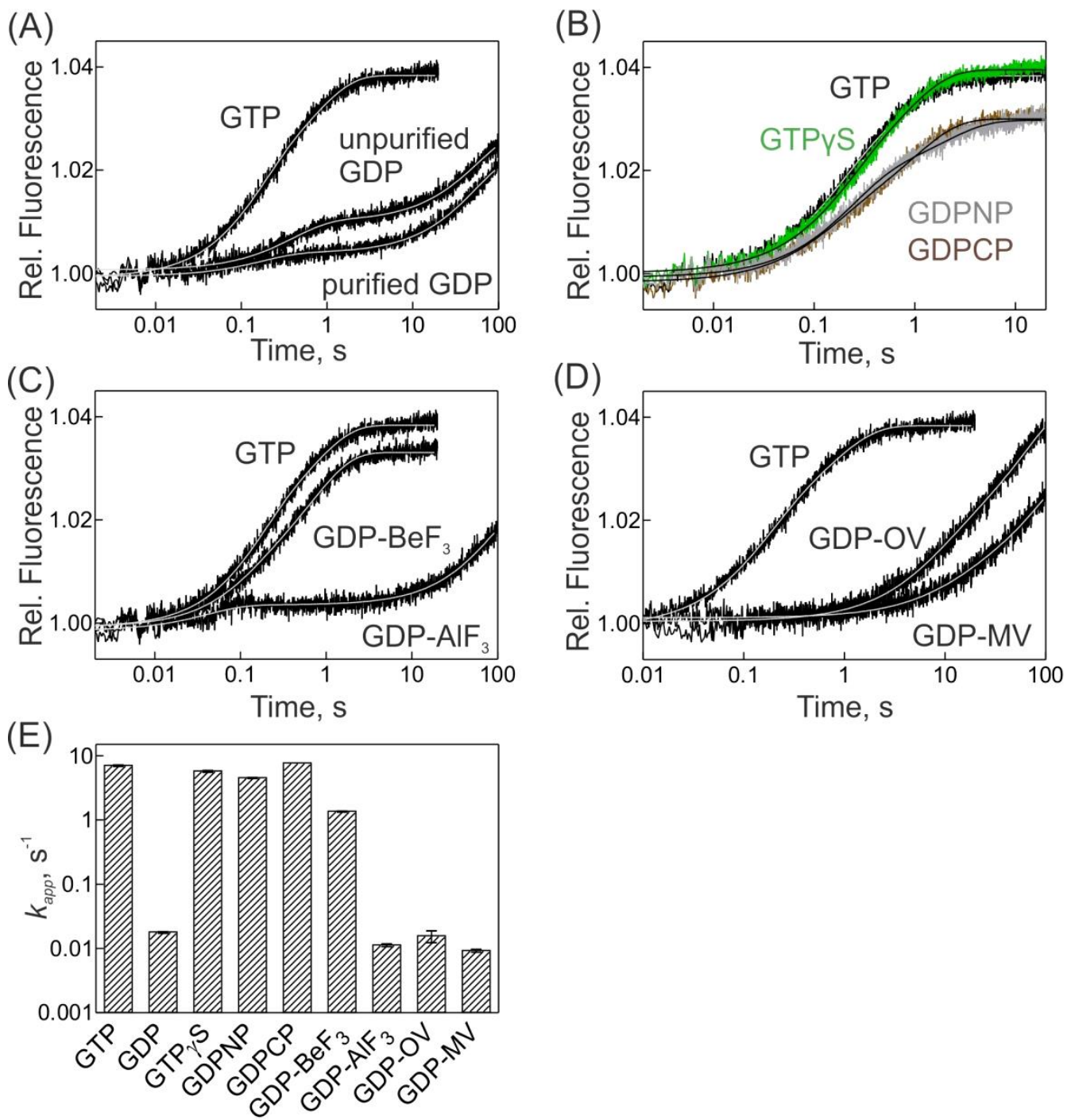

Figure 12. Effect of different guanosine nucleotide analogs on subunit association. 30 IC $(0.05 \mu \mathrm{M})$ was rapidly mixed with 50 S subunits $(0.25 \mu \mathrm{M})$ in a stopped-flow apparatus and the change in LS upon subunit association was monitored. (A) Time courses of subunit association in the presence of GTP, commercially-purchased GDP and purified GDP. (B) Time courses of subunit association in the presence of non-hydrolysable GTP analogs GTPYS, GDPNP and GDPCP. (C) Time courses of subunit association in the presence of the presumed ground- and transition-state GTP analogs - GDP-BeF 3 and GDP-AlF 3 , respectively. (D) Time courses of subunit association in the presence of post-hydrolysis state GTP analogs - GDP—ortho-vanadate and GDP—meta-vanadate. (E) Apparent rates of the predominant phase derived from double-exponential fitting of time courses in (A)-(D); error bars represent standard error of the fit.

In the presence of non-hydrolysable GTP analogs such as GTPYS and GDPNP, rapid subunit joining was observed, like in case of GTP (Fig. 12B), in agreement with earlier reports (Antoun et al., 2003; Antoun et al., 2004; Qin et al., 2009, Tsai et al., 2012). However, 
in contrast to a previous study which reported slower subunit joining in the presence of the non-hydrolysable GTP analog, GDPCP (Grigoriadou et al., 2007a), no difference in the rate of subunit association was seen. These results confirm that GTP hydrolysis by IF2 is not required during early stages of initiation such as $30 \mathrm{SIC}$ formation and subunit joining (Anderson et al., 1967; Antoun et al., 2003; Qin et al., 2009; Tsai et al., 2012).

The effect of different transition-state GTP analogs on $50 \mathrm{~S}$ joining was also checked. Beryllium fluoride $\left(\mathrm{BeF}_{3}\right)$ and aluminium fluoride $\left(\mathrm{AlF}_{3}\right)$ behave as phosphate analogs and bind, along with GDP, in the nucleotide binding pocket of IF2 to mimic different pre-hydrolysis GTP transition states. For a variety of GTP-binding proteins, $\mathrm{BeF}_{3}$ mimics the ground state of $\mathrm{Y}$-phosphate and confers a GTP-like conformation, while $\mathrm{AlF}_{3}$ represents a transition state of the $\mathrm{y}$-phosphate during GTP hydrolysis (reviewed in (Golicnik, 2010)). Vanadium $\left(\mathrm{VO}_{4}{ }^{3-}\right.$ ), in the form of ortho- and meta-vanadate, is a close structural and chemical mimic of phosphate which can bind to the nucleotide binding site of IF2, along with GDP, and represents a posthydrolysis GDP_Pi state (Goodno, 1982). Rapid subunit joining was observed in the presence of GDP and $\mathrm{BeF}_{3}$ but not when GDP and $\mathrm{AlF}_{3}$ were used (Fig. 12C). The time course of $\mathrm{LS}$ recorded in the presence of $\mathrm{AlF}_{3}$ was similar to that observed in the case of GDP alone, suggesting that either $\mathrm{AlF}_{3}$ did not bind to IF2 or the $\mathrm{AlF}_{3}$-bound transition state of IF2 does not promote subunit joining. In presence of GDP and ortho-vanadate (Fig. 12D), the kinetics of LS was biphasic, with the first, minor phase occurring at $0.13 \mathrm{~s}^{-1}$. The second, major phase was slow $\left(0.01 \mathrm{~s}^{-1}\right)$, similar to that seen in the presence of GDP alone. When meta-vanadate was used instead, only the slow phase was observed.

In summary, these results show that the IF2 conformation, when bound to pre-hydrolysis state analogs such as GTP, GTPYS, GDPNP, GDPCP and GDP-BeF $F_{3}$, promotes rapid subunit joining $\left(5 \mathrm{~s}^{-1}\right)$ (Fig. 12E). On the other hand, subunit joining is up to 500 -fold slower when IF2 is bound to transition-state and post-hydrolysis state analogs such as GDP-AlF GDP_ortho-vanadate, GDP — meta-vanadate and GDP alone, indicating that one important role of GTP during translation initiation is to confer a productive conformation of IF2 on the $30 \mathrm{~S} \mathrm{IC}$ which is conducive to rapid subunit association. 


\subsection{Dynamics of IF2 on the 70S IC}

Post-subunit joining events such as GTPase activation of IF2, the loss of direct interaction between fMet-tRNA ${ }^{\text {fMet }}$ and C2-domain of IF2, as well as the dissociation of GDP from IF2 have not yet been monitored. It is also not clear whether IF2 is released from the ribosome after GTP hydrolysis. Finally, the role of GTP hydrolysis by IF2 during initiation, as studied using different non-hydrolysable GTP analogs, has yielded inconsistent results depending on the nucleotide and the source of initiation components (Allen et al., 2005; Antoun et al., 2003; Grigoriadou et al., 2007a; Myasnikov et al, 2005; Qin et al., 2009; Tomsic et al., 2000). In this section, IF2-dependent reactions which take place after subunit joining were monitored using rapid kinetics and their dependence on GTP hydrolysis and the presence of IF1 and IF3 was investigated.

\subsubsection{GTPase activation and dissociation of IF2}

FRET between a fluorescent GTP analog, mant-GTP and the intrinsic Trp residue of IF2 (Fabbretti et al., 2012; Milon et al., 2007) was followed to study the conformational dynamics of the IF2 G-domain. The kinetics of mant-GTP binding to and dissociation from IF2, reported previously, indicated that the affinity of IF2 for mant-GTP is in the same range as that for non-fluorescent GTP (Fabbretti et al., 2012; Simonetti et al., 2013). Upon 50 S joining to $30 \mathrm{~S}$ IC formed with mant-GTP, a biphasic FRET change was observed. A similar FRET signal was previously reported in the case of EF-Tu binding to post-translocation ribosome complexes (Maracci et al., 2014; Rodnina et al., 1994). Thus, in analogy with EF-Tu, the first upward phase was assigned to GTPase activation (Fig. 13A), during which residues in the G-domain of the factor rearrange themselves into a conformation which is primed to catalyze GTP hydrolysis, while the second downward phase most likely reflects IF2 dissociation from the $70 \mathrm{~S}$ complex, although it cannot be excluded that the phase may represent the release of the nucleotide from IF2 or a conformational rearrangement of the factor upon dissociation from the ribosome. In the absence of $50 \mathrm{~S}$ subunits (only buffer), a slow downward amplitude signal change was observed (Fig. 13B). Thus, the fitting of the buffer trace was subtracted from the time courses of all experiments conducted using mant-GTP. 
(A)

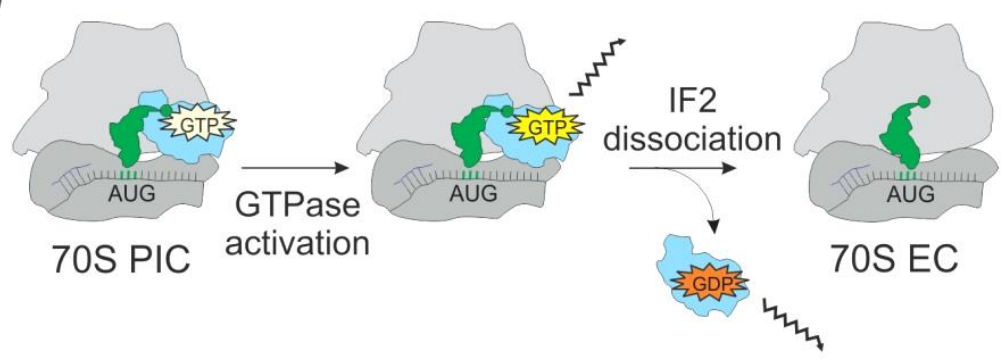

(B)

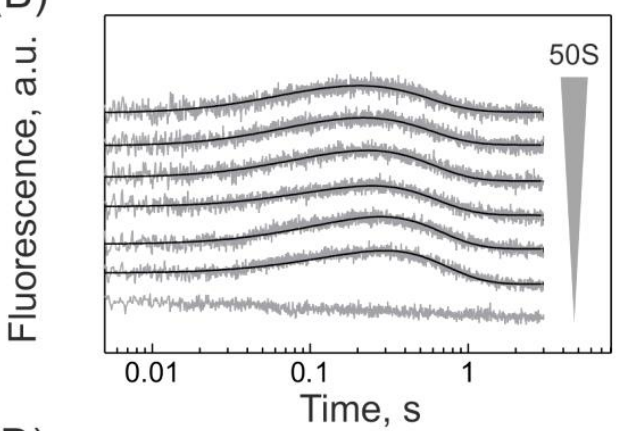

(D)

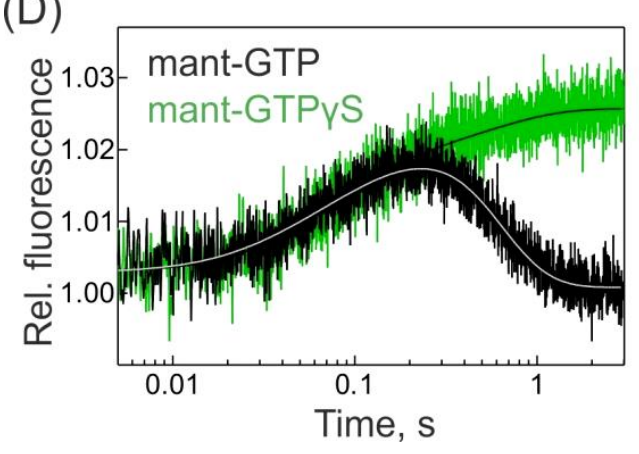

(C)

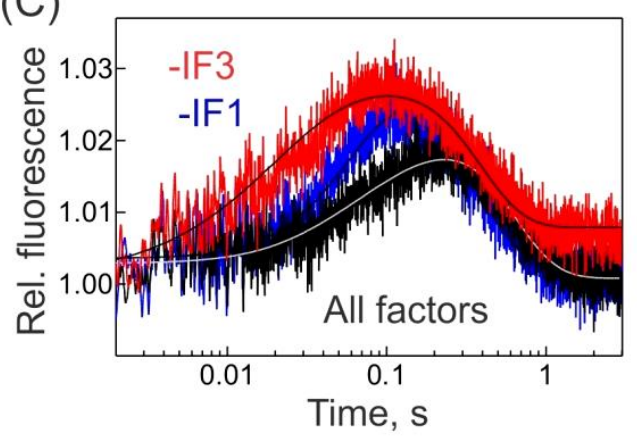

Figure 13. GTPase activation and dissociation of the IF2 during 70S IC formation. $30 \mathrm{~S}$ IC $(0.15 \mu \mathrm{M})$, formed with mant-GTP was rapidly mixed with 50 S subunits in a stopped-flow apparatus and the change in FRET was used to monitor GTPase activation and IF2 dissociation during 70 S IC formation. (A) Schematic of GTPase activation and dissociation of IF2 from the 70S complex. (B) Time courses of FRET upon rapidly mixing $30 \mathrm{~S}$ IC with increasing concentrations of $50 \mathrm{~S}$ subunits $(0.45-1.5 \mu \mathrm{M})$. Control measurements were performed in the absence of $50 \mathrm{~S}$ subunits. (C) Time courses of FRET upon rapidly mixing 30 S IC formed in the presence of all factors and absence of IF1 or IF3 with 50S subunits (1 $\mu \mathrm{M})$. (D) Time courses of FRET upon rapidly mixing $30 \mathrm{~S} \mathrm{IC}$, formed in the presence of mantGTP or mant-GTPYS, with $50 \mathrm{~S}$ subunits $(1 \mu \mathrm{M})$.

Subunit joining was followed by GTPase activation (apparent rate $-9 \mathrm{~s}^{-1}$ ) and subsequent IF2 dissociation $\left(2.5 \mathrm{~s}^{-1}\right)$. In the absence of IF1 or IF3, GTPase activation appeared to be faster (14 $\mathrm{s}^{-1}$ and $27 \mathrm{~s}^{-1}$, respectively) (Fig. 13C), while the apparent rate of IF2 dissociation from the 70S complex was not affected (Table 1). When mant-GTP was replaced by mantGTPYS, only the first phase of FRET change, depicting GTPase activation was observed 
(similar to the case of EF-Tu (Daviter et al., 2003)), suggesting that conformational rearrangements of the G-domain can take place in the presence of a non-hydrolysable GTP analog (Fig. 13D). The second phase was blocked, in agreement with previous reports that IF2 cannot dissociate from the 70S ribosome in the absence of GTP hydrolysis (Allen et al., 2005; Antoun et al., 2003; Laalami et al., 1994; Lockwood et al., 1972; Luchin et al., 1999; Myasnikov et al., 2005).

\subsubsection{Pi release from IF2}

50 S subunit joining to the $30 \mathrm{~S}$ IC triggers GTP hydrolysis by IF2 into GDP and Pi (Grigoriadou et al., 2007a; Tomsic et al., 2000). Pi release from IF2 was followed by the increase in fluorescence which occurred upon its subsequent rapid binding to a fluorescent derivative of phosphate binding protein, MDCC-PBP (Fig. 14A) (Brune et al., 1994; Tomsic et al., 2000).

(A)

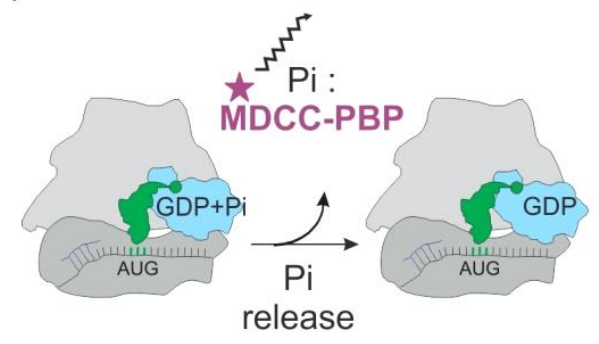

(B)

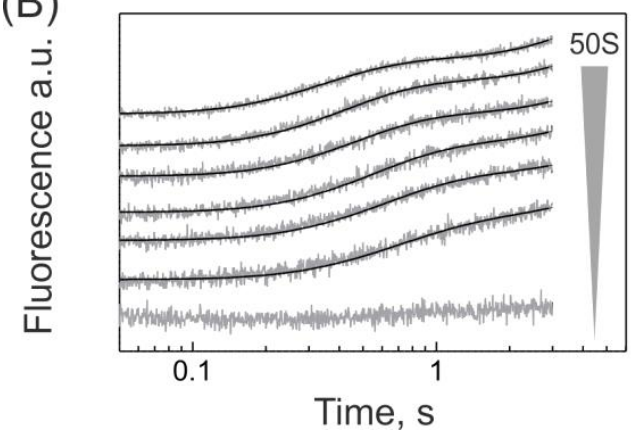

(C)

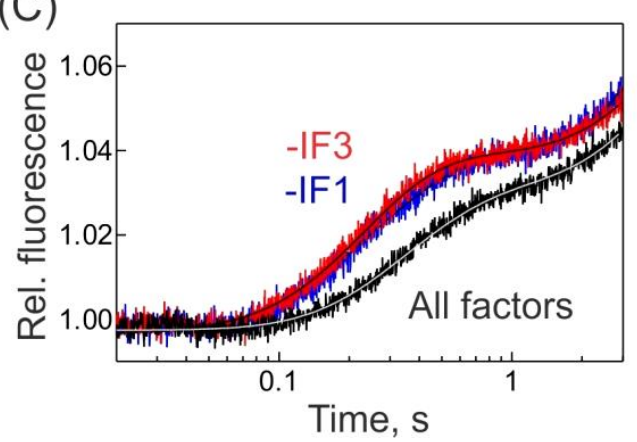

Figure 14. Pi release from IF2 on the 70S IC. 30 S IC $(0.05 \mu \mathrm{M})$ was rapidly mixed with 50 S subunits in a stopped-flow apparatus and the fluorescence change of PBP-MDCC upon its rapid binding to free Pi was used to monitor Pi release from IF2. (A) Schematic of Pi release from IF2 during 70S IC formation. (B) Time courses of Pi release from IF2 upon rapidly mixing $30 \mathrm{~S} \mathrm{IC}$ with increasing concentrations of $50 \mathrm{~S}$ subunits $(0.15 \mu \mathrm{M}-1 \mu \mathrm{M})$. Control measurements were performed in the absence of $50 \mathrm{~S}$ subunits. (C) Time courses of $\mathrm{Pi}$ release from IF2 upon rapidly mixing $30 \mathrm{~S} \mathrm{IC} \mathrm{formed} \mathrm{in} \mathrm{the} \mathrm{presence} \mathrm{of} \mathrm{all} \mathrm{factors} \mathrm{and}$ absence of IF1 or IF3 with 50 S subunits $(1 \mu \mathrm{M})$. 
When 30 S IC was rapidly mixed with 50 S subunits, Pi release took place after a lag phase (75 ms) (Fig. 14B). Due to the significant delay phase, the time courses could not be fit with an exponential function to calculate the apparent rate constant of the reaction. The initial increase in fluorescence was followed by a slow step $\left(0.01 \mathrm{~s}^{-1}\right)$, representative of the multiple turnover events of GTP hydrolysis and Pi release which occur due to the rebinding of IF2 to mature $70 \mathrm{~S} \mathrm{IC.} \mathrm{In} \mathrm{the} \mathrm{absence} \mathrm{of} 50 \mathrm{~S}$ subunits, no change in fluorescence was observed due to the low levels of intrinsic GTPase activity of IF2 in the time range measured (Huang et al., 2010; Severini et al., 1991). When complexes lacking IF1 or IF3 were used, the rate of Pi release appeared slightly faster (Fig. 14C), which may be attributed to the faster rates of subunit joining on these complexes.

\subsubsection{Release of fMet-tRNA ${ }^{\mathrm{fMet}}$ from the C2-domain of IF2}

Direct interaction between the 3' CCA-end of tRNA ${ }^{\text {fMet }}$ and the C2-domain of IF2 on the ribosome was monitored using Bodipy (Bpy) FL- labeled Met-tRNA ${ }^{\mathrm{fMet}}$ (Holtkamp et al., 2014) An increase in Bpy-Met-tRNA ${ }^{\mathrm{fMet}}$ fluorescence was observed upon its recruitment to the $30 \mathrm{~S}$ PIC by IF2. No fluorescence change occurred in the absence of IF2, 30S subunit or when a truncated mutant of IF2 lacking the C2-domain (IF2 $\triangle$ C2) was used instead (Fig. 15A). The chase of Bpy-Met-tRNA ${ }^{\text {fMet }}$ from the $30 \mathrm{~S} \mathrm{IC} \mathrm{with} \mathrm{non-fluorescent} \mathrm{fMet-tRNA}{ }^{\text {fMet }}$ was almost undetectable (Fig. 15B), in agreement with previous reports that codon-anticodon interaction with the mRNA AUG codon greatly stabilizes tRNA ${ }^{\text {fMet }}$ on the ribosome (Milon et al., 2012).
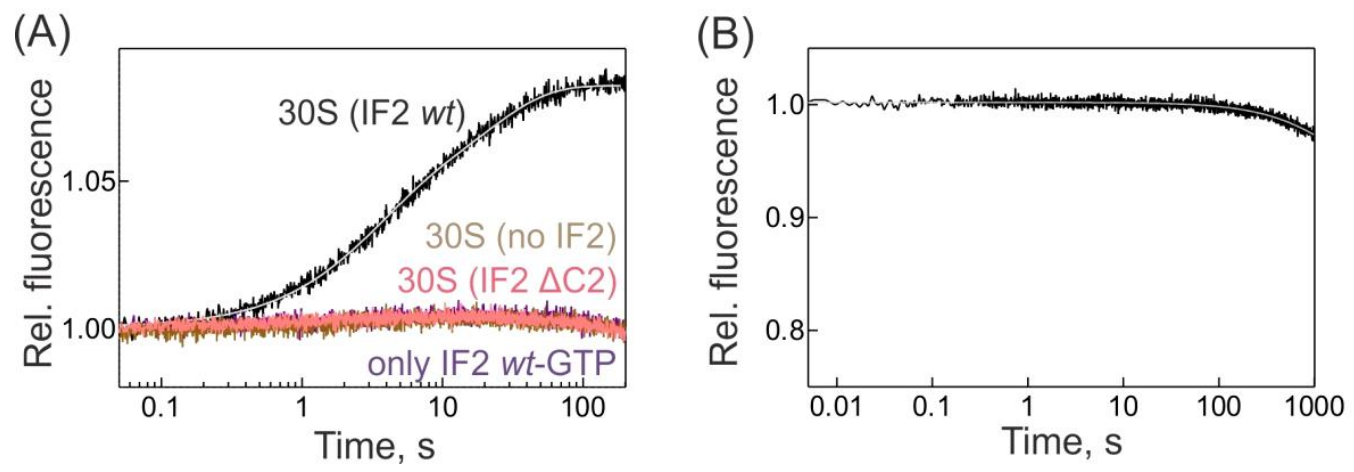

Figure 15. Binding and dissociation kinetics of Bpy-Met-tRNA ${ }^{\mathrm{fMet}}$ on the $30 \mathrm{~S}$ IC. Reactions were performed by rapidly mixing indicated components in a stopped-flow apparatus and following the fluorescence changes of Bpy-Met-tRNA ${ }^{\text {tMet }}$. (A) Time courses Bpy-Met-tRNA $A^{\text {fMet }}$ binding to 30 S PIC (lacking fMet-tRNA ${ }^{\text {fMet }}$ ) or free IF2-GTP. (B) Time courses of Bpy-Met-tRNA ${ }^{\text {fMet }}$ dissociation from the $30 \mathrm{~S} \mathrm{IC} \mathrm{upon} \mathrm{chase} \mathrm{with} \mathrm{10-fold} \mathrm{excess} \mathrm{of}$ non-fluorescent fMet-tRNA ${ }^{\mathrm{fMet}}$. 
Upon 50 S joining to $30 \mathrm{~S} \mathrm{IC} \mathrm{formed} \mathrm{with} \mathrm{Bpy-Met-tRNA}{ }^{\mathrm{fMet}}$, a decrease in fluorescence was observed, which can be attributed to the release of tRNA ${ }^{\text {fMet }}$ from IF2 during 70S IC formation (Fig. 16A-B). The time courses were monitored at increasing 50S subunit concentrations and the reaction was preceded by a $\sim 100$ ms delay phase. The tRNA ${ }^{\text {fMet }}$ release from IF2 appeared faster when IF1 or IF3 was lacking from the complex (Fig. 16C). GTP hydrolysis was essential for tRNA ${ }^{\text {fMet }}$ release from the C2-domain of IF2 because replacing GTP with GTPYS or GDPCP completely abolished the reaction (Fig. 16D).

(A)

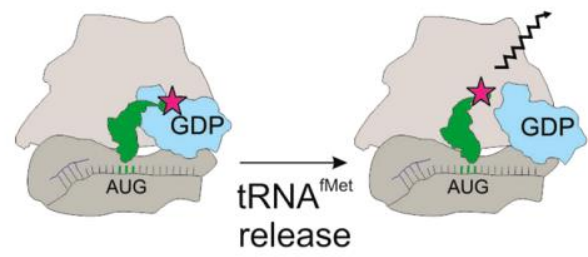

(C)

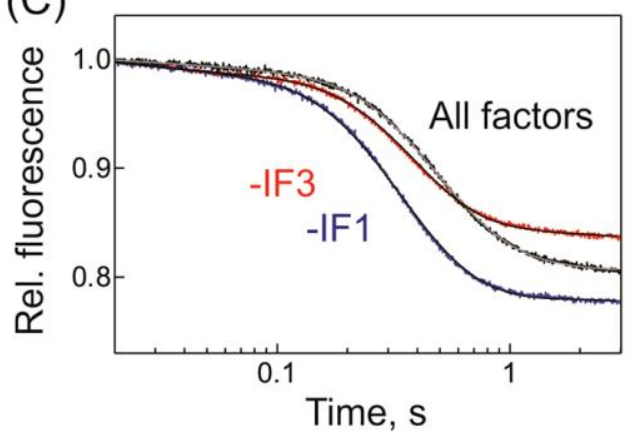

(B)

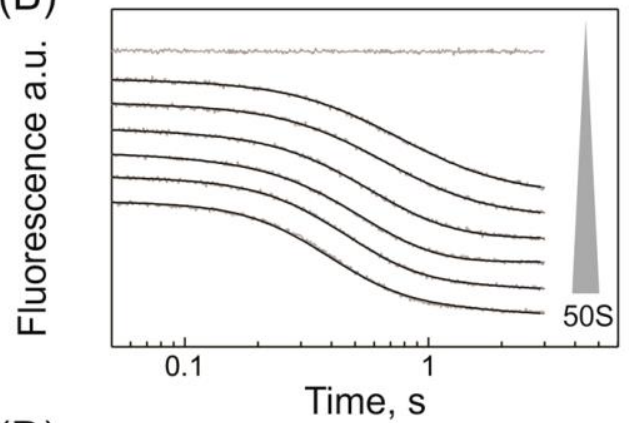

(D)

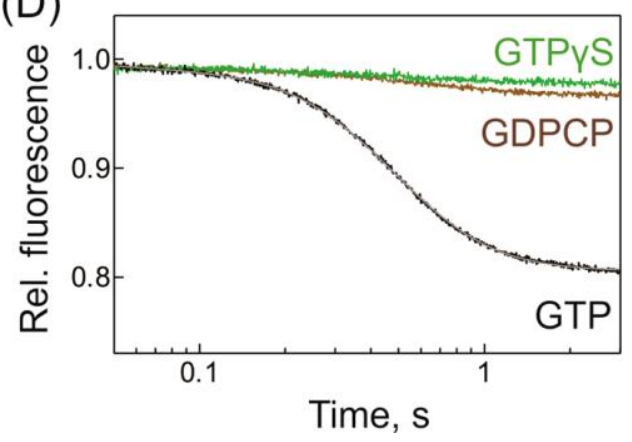

Figure 16. Release of tRNA ${ }^{\text {fMet }}$ from IF2 during 70 S IC formation. $30 S$ IC $(0.05 \mu \mathrm{M})$, formed with Bpy-Met-tRNA ${ }^{\text {fMet }}$ was rapidly mixed with $50 \mathrm{~S}$ subunits in a stopped-flow apparatus and the decrease in Bpy fluorescence was used to monitor tRNA ${ }^{\text {fMet }}$ dissociation from IF2 during 70 S IC formation. (A) Schematic of Bpy-Met-tRNA ${ }^{\text {tMet }}$ dissociation from IF2 during 70 S IC formation. (B) Time courses of Bpy-Met-tRNA ${ }^{\text {fMet }}$ dissociation from IF2 upon rapidly mixing $30 \mathrm{~S} I C$ with increasing concentrations of $50 \mathrm{~S}$ subunits $(0.15 \mu \mathrm{M}-1 \mu \mathrm{M})$. Control measurements were performed in the absence of $50 \mathrm{~S}$ subunits. (C) Time courses of Bpy-Met-tRNA ${ }^{\text {tMet }}$ dissociation from IF2 upon rapidly mixing $30 \mathrm{~S} \mathrm{IC} \mathrm{formed} \mathrm{in} \mathrm{the} \mathrm{presence} \mathrm{of}$ all factors and absence of IF1 or IF3 with 50 S subunits $(1 \mu \mathrm{M})$. (D) Time courses of Bpy-MettRNA ${ }^{\text {tMet }}$ dissociation from IF2 upon rapidly mixing 30 S IC, formed in the presence of GTP, GTPYS or GDPCP, with 50 S subunits $(1 \mu \mathrm{M})$.

\subsubsection{Dissociation of GDP from IF2}

The fluorescence changes of Bpy-GTP and Bpy-GDP were used to monitor the direct interaction of the nucleotide with IF2. Binding of Bpy-GDP to free IF2 resulted in a single- 
exponential increase in fluorescence, the rates of which saturated $\left(10 \mathrm{~s}^{-1}\right)$ at high IF2 concentrations, indicative of a monomolecular rearrangement which follows the binding step (Fig. 17A-B). Binding of Bpy-GDP to 30S-bound IF2 also showed a similar fluorescence change $\left(10 \mathrm{~s}^{-1}\right)$. In contrast, upon binding of Bpy-GTP to IF2 on the 30S IC, a biphasic increase in fluorescence was observed with the slow $\left(0.05 \mathrm{~s}^{-1}\right)$ phase accounting for $>90 \%$ of the amplitude change and a rapid step with a very small amplitude (Fig. 17C). Binding of Bpy-GTP to free IF2 displayed only the first small phase which occurred with the rate of $10 \mathrm{~s}$ ${ }^{1}$ (similar to Bpy-GDP), indicating that this phase may arise due to a slight contamination of the Bpy-GTP preparation with Bpy-GDP or a conformational rearrangement that is common to the binding of both nucleotides to IF2. Bpy-GTP and Bpy-GDP could be chased from $30 \mathrm{~S}$ IC-bound IF2 with the addition of excess GTP (Fig. 17D), resulting in a $12 \%$ decrease of fluorescence. The dissociation rate constants of Bpy-GDP and Bpy-GTP release from IF2, determined from the chase experiments were $10 \mathrm{~s}^{-1}$ and $0.01 \mathrm{~s}^{-1}$, respectively.
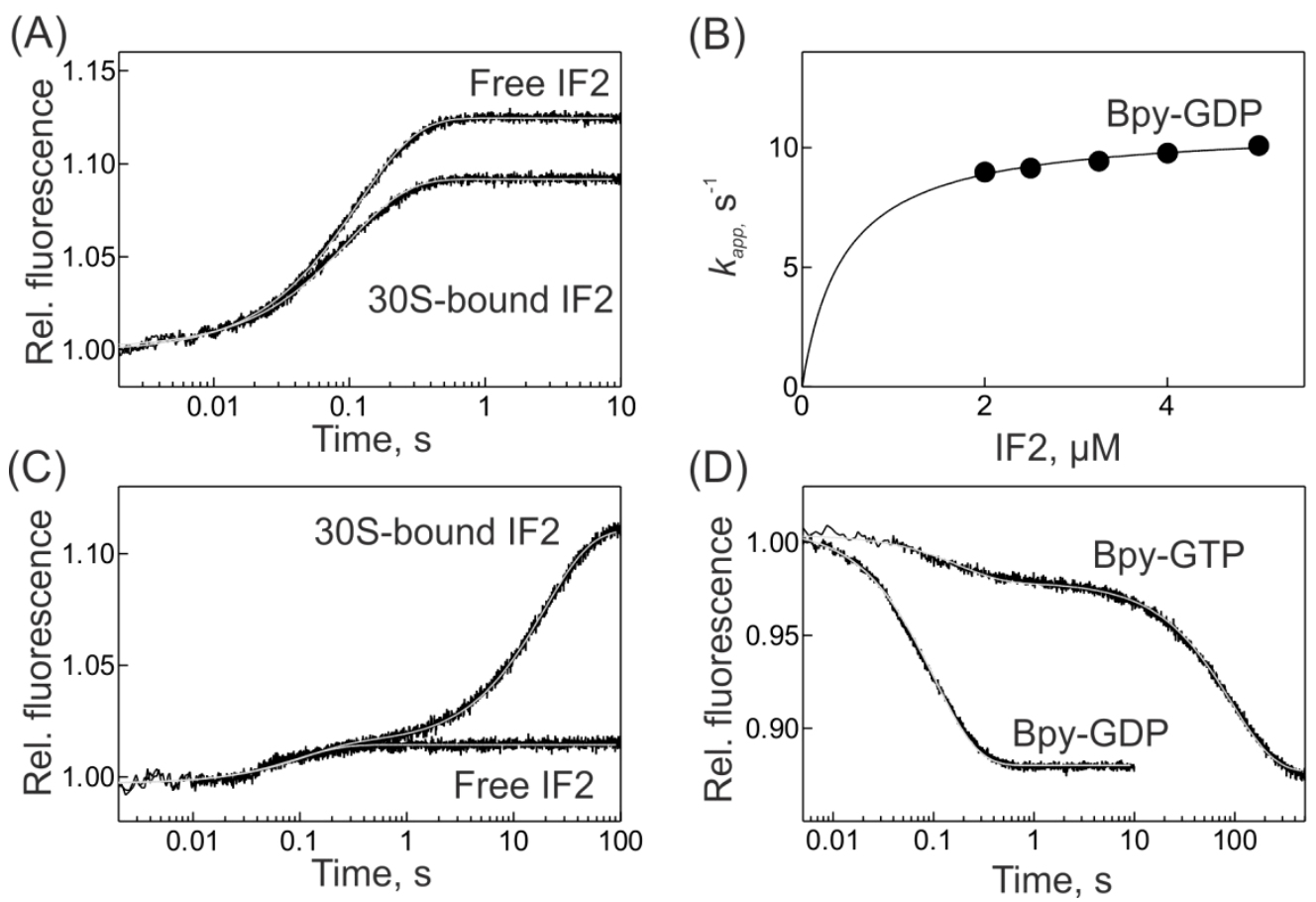

Figure 17. Binding and dissociation kinetics of Bpy-GTP and Bpy-GDP. Reactions were performed by rapidly mixing components in a stopped-flow apparatus and following the fluorescence changes of the Bpy-labeled guanosine nucleotide. (A) Time courses of BpyGDP binding to free IF2 or, IF2 bound to 30 S PIC (formed in the absence of any nucleotide). (B) Apparent rates of Bpy-GDP $(2 \mu \mathrm{M})$ binding to increasing concentrations of free IF2. Rates and standard errors (error bars smaller than symbol size are not visible) were derived from single-exponential fitting of time courses. (C) Time courses of Bpy-GTP binding to free IF2 or, IF2 bound to 30S PIC. (D) Time courses of Bpy-GTP or Bpy-GDP release from IF2 bound on the 30S IC, upon chase with 125-fold excess of non-fluorescent GTP. 
When Bpy-GTP was used to form the 30S IC, the Bpy fluorescence decreased after a lag phase of $\sim 200 \mathrm{~ms}$ following the $50 \mathrm{~S}$ subunit joining (Fig. 18B). The amplitude change (10\%) was similar to that observed during the nucleotide binding and dissociation (chase) reactions. Hence, we concluded that the decrease in fluorescence of Bpy-GTP observed upon 70S IC formation corresponds to the release of Bpy-GDP from IF2 after hydrolysis of Bpy-GTP (Fig. 18A). The absence of IF1 or IF3 did not affect the reaction (Fig. 18C).

(A)
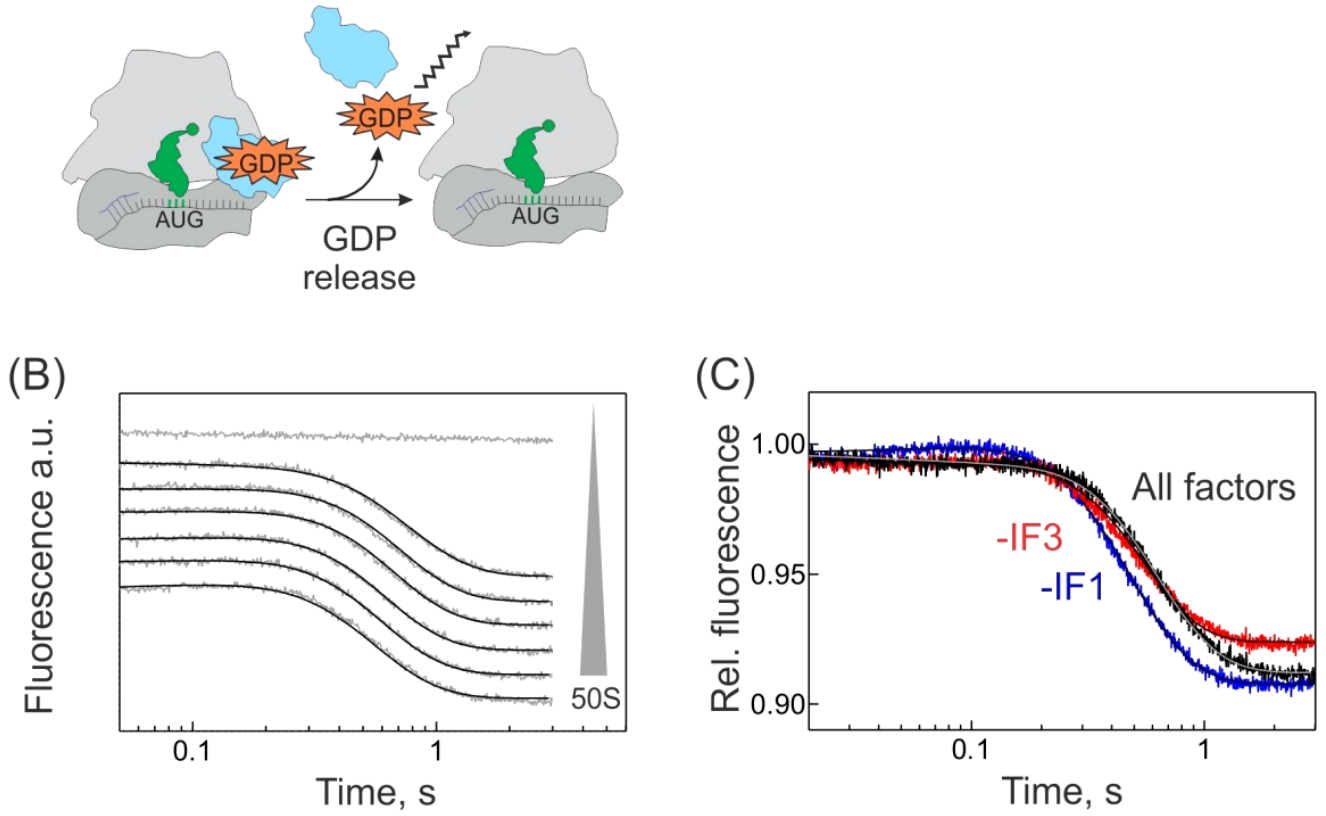

Figure 18. Release of GDP from IF2 during 70S IC formation. 30S IC $(0.1 \mu \mathrm{M})$ formed with Bpy-GTP was rapidly mixed with $50 \mathrm{~S}$ subunits in a stopped-flow apparatus and the decrease in Bpy fluorescence was used to monitor GDP release from IF2 during 70S IC formation. (A) Schematic of Bpy-GDP release from IF2 during 70 S IC formation. (B) Time courses of Bpy-GDP release from IF2 upon rapidly mixing 30S IC with increasing concentrations of $50 \mathrm{~S}$ subunits $(0.3-2 \mu \mathrm{M})$. Control measurements were performed in the absence of $50 \mathrm{~S}$ subunits. (C) Time courses of Bpy-GDP release from IF2 upon rapidly mixing $30 \mathrm{~S} \mathrm{IC} \mathrm{formed} \mathrm{in} \mathrm{the} \mathrm{presence} \mathrm{of} \mathrm{all} \mathrm{factors} \mathrm{and} \mathrm{absence} \mathrm{of} \mathrm{IF1} \mathrm{or} \mathrm{IF3,} \mathrm{with} 50 \mathrm{~S}$ subunits $(1 \mu \mathrm{M})$.

\subsubsection{Participation of IF2 in the $2^{\text {nd }}$ round of initiation}

To monitor the direct interaction of IF2 with the ribosome, a fluorescence-labeled mutant of the factor, IF2 $2_{757}(\mathrm{Al} \times 555)$ (Dr. Cristina Maracci - PhD thesis) was used. Binding of IF2 257 (Alx555) to the 30 S complex resulted in a triphasic change in fluorescence (Fig. 19A). No fluorescence change was observed in the absence of 30S subunits, indicating that the fluorescence change did not arise from the interaction of IF2 with GTP or fMet-tRNA ${ }^{\text {fMet }}$, off 
the ribosome. In the presence of $30 \mathrm{~S}$ subunits, but in the absence of nucleotide or fMet$\mathrm{tRNA}^{\mathrm{fMet}}$, only the first phase was observed (Fig.19B-C). When GTP was replaced by GDP, the amplitude of the third phase was lowered significantly. These results indicate that the first phase of fluorescence change depicts direct binding of IF2 to the 30S subunit $\left(20 \mathrm{~s}^{-1}\right)$, while the following phases represent conformational changes of the factor induced by its interaction with GTP and fMet-tRNA ${ }^{\mathrm{fMet}}$. The third phase most likely depicts the conversion of IF2 into an 'active' conformational state, which promotes rapid subunit association and does not take place in the presence of GDP. When IF2 $2_{757}$ (Alx555) was chased from the 30 S IC with a 20 -fold excess of non-fluorescent IF2, a biphasic change in fluorescence was observed which may represent the reversal of IF2 conformational change, followed by dissociation of the factor from the 30S complex (Fig.19D). Further experiments, in the presence and absence of different initiation components, would help characterize the two phases in more detail.
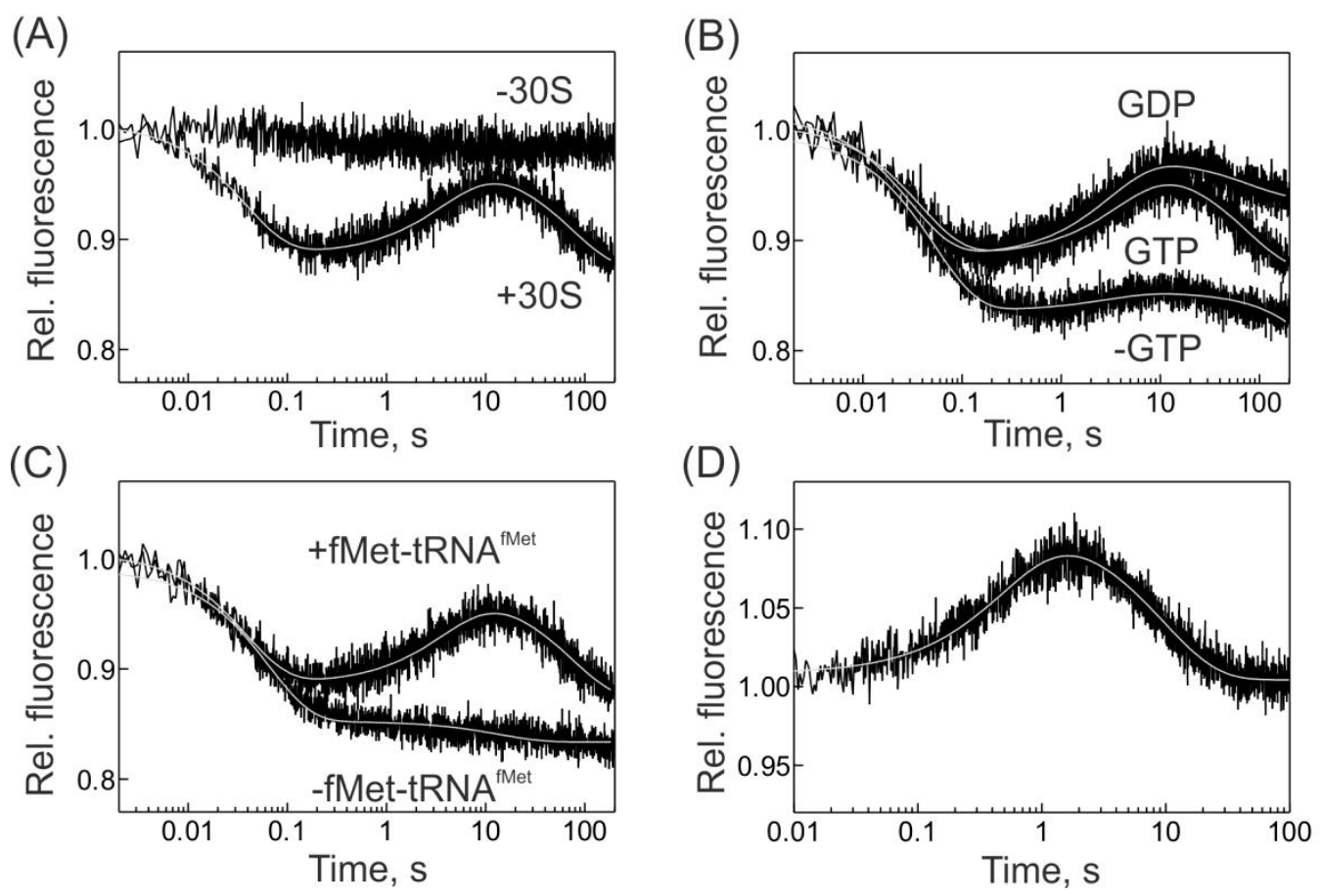

Figure 19. Binding and dissociation kinetics of IF2 ${ }_{757}(\mathrm{Al} \times 555)$ on the $30 \mathrm{~S} \mathrm{IC}$. Reactions were performed by rapidly mixing indicated components in a stopped-flow apparatus and following the fluorescence changes of IF2 757 (Alx555). (A) Time courses IF2 757 (Alx555) binding to 30S PIC (lacking IF2). (B) Time courses IF2 ${ }_{757}$ (Alx555) binding to 30 S PIC in the presence of GTP, GDP or no nucleotide. (C) Time courses IF2 ${ }_{757}$ (Alx555) binding to 30 S PIC in the presence or absence of fMet-tRNA ${ }^{\text {iMet }}$. (D) Time courses of IF2 ${ }_{757}$ (Alx555) release from the $30 \mathrm{~S} \mathrm{IC}$, upon chase with 20 -fold excess of non-fluorescent IF2. 
After completing a single round of initiation, IF2 presumably dissociates from the $70 S$ complex to participate in further initiation events (Fig. 20A). When 30 S IC was formed with an excess of $30 S$ subunits over IF $2_{757}($ Alx555), 50 S joining led to a $\sim 15 \%$ decrease in fluorescence preceded by a delay phase (Fig. 20B). A similar signal change was previously observed upon IF2 757 (Alx555) binding to the 30 S complex suggesting that after a delay phase of $\sim 200 \mathrm{~ms}$. which represents the time required for completing the first round of initiation, IF2 ${ }_{757}($ Alx555) is released from the 70 S IC and binds to the free 30 S subunits in solution. The absence of IF1 and IF3 did not affect the reaction, although a small, undefined decrease in fluorescence preceding the main phase was observed, which may be caused by 50 S joining to 30 S IC containing a differently positioned IF2 (Fig. 20C). GTP hydrolysis was a prerequisite for IF2 dissociation and the reaction was abolished with GTPYS (Fig. 20D).
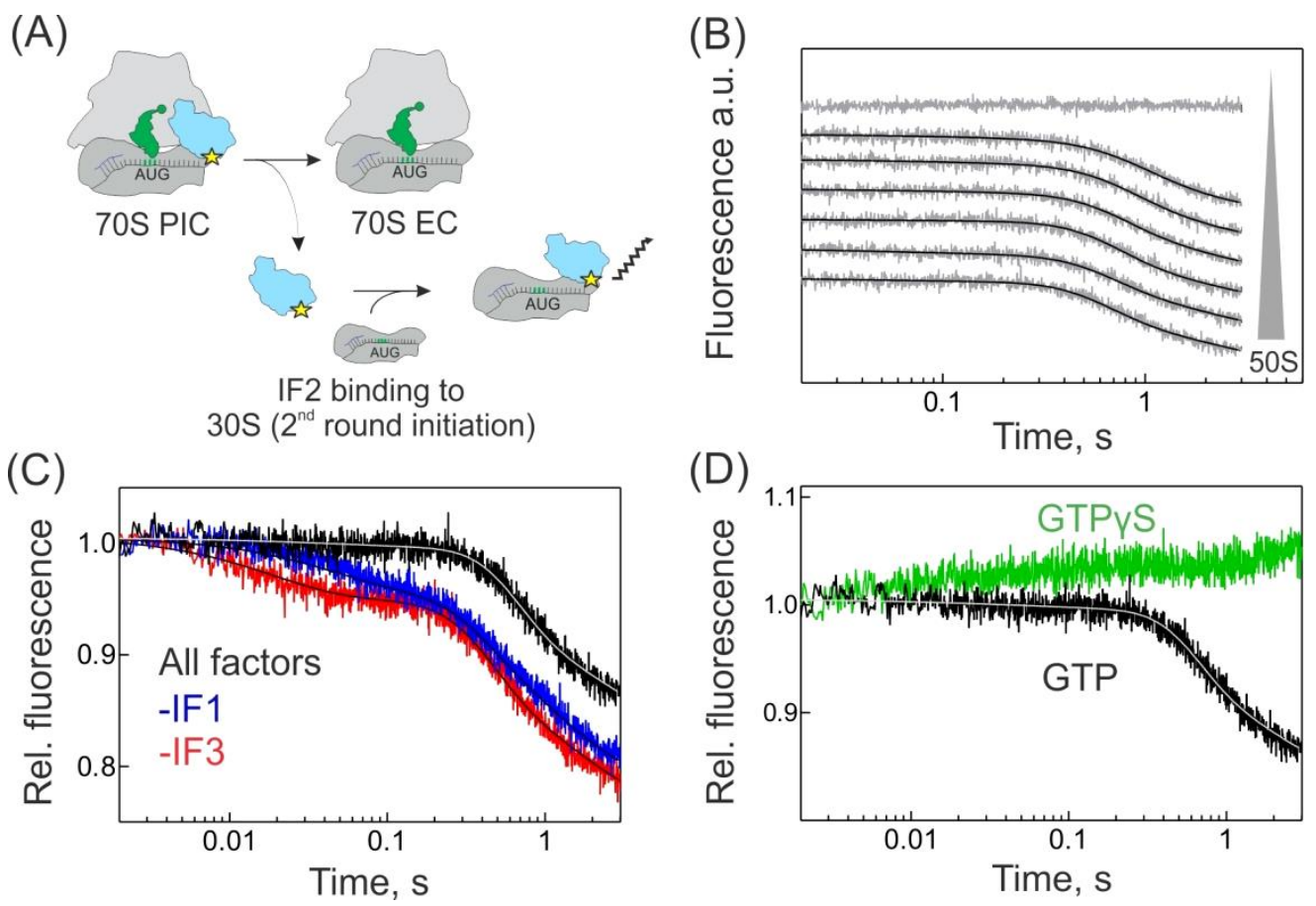

Figure 20. IF2 binding to 30 S PIC during the $2^{\text {nd }}$ round of initiation. $30 S$ IC $(0.05 \mu M)$, formed in the presence of 30 S subunits $(0.1 \mu \mathrm{M})$ and limiting amounts of IF2 ${ }_{757}$ (Alx555) $(0.05$ $\mu \mathrm{M})$, was rapidly mixed with $50 \mathrm{~S}$ subunits in a stopped-flow apparatus and the fluorescence change of $\mathrm{FF}_{757}(\mathrm{Al} \times 555)$ was used to monitor IF2 entry into the $2^{\text {nd }}$ round of initiation. (A) Schematic of IF2 dissociation from the 70S IC during the $1^{\text {st }}$ round of initiation and subsequent binding to $30 \mathrm{~S}$ PIC during the $2^{\text {nd }}$ round of initiation. (B) Time courses of IF2 ${ }_{757}$ (Alx555) binding to $30 \mathrm{~S}$ PIC $\left(2^{\text {nd }}\right.$ round) upon mixing $30 \mathrm{~S}$ IC with increasing concentrations of $50 \mathrm{~S}$ subunits $(0.25-1 \mu \mathrm{M})$. Control measurements were performed in the

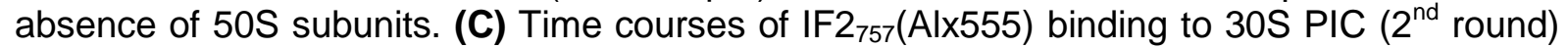
upon mixing 30 S IC formed in the presence of all factors and absence of IF1 or IF3 with 50S subunits $(1 \mu \mathrm{M})$. (D) Time courses of IF2 ${ }_{757}(\mathrm{Alx555})$ binding to $30 \mathrm{~S}$ PIC (2 ${ }^{\text {nd }}$ round) upon rapidly mixing 30 S IC formed in the presence of GTP or GTPYS with 50 S subunits $(1 \mu \mathrm{M})$. 


\subsection{Dynamics of IF1 on the 70S IC}

The binding and dissociation kinetics of IF1 on the 30 S IC, as well as the interplay of IF1 with the other two factors, have been previously studied using fluorescence-labeled initiation components and rapid kinetic approaches (Milon et al., 2012). Until now, however, no studies have been conducted to investigate the dynamics of IF1 on the 70S IC. In the following section, dynamics of IF1 on the 70S complex were monitored with the help of two reporters; the fluorescence changes of $\mathrm{IF}_{4}(\mathrm{Al} \times 555)$, as well as the loss of FRET between $30 \mathrm{~S}_{\mathrm{S}_{13}}(\mathrm{Al} \times 488)$ and IF1 ${ }_{4}$ (Atto540Q). Because IF1 is a relatively small, tightly folded protein, it is unlikely that the fluorescence intensity change of $\mathrm{IF}_{4}(\mathrm{Al} \times 555)$ reflects a conformational change of the factor itself. Rather, it may reflect an alteration in the environment of the reporter owing to a conformational rearrangement of the complex or establishment of alternative IF1 contacts (Qin and Fredrick, 2009) (in the following text, this step is referred to as 'change of IF1 environment'). The timing of $\mathrm{IF} 1_{4}(\mathrm{Al} \times 555)$ fluorescence change preceded the change in FRET between IF1 and 30S. Hence, it is likely that the former observable represents changes in IF1 environment after subunit association, while the latter observable depicts the subsequent dissociation of the factor from the $70 \mathrm{~S}$ complex.

\subsubsection{Change in IF1 environment after subunit joining}

When $\mathrm{IF}_{4}(\mathrm{Al} \times 555)$ was rapidly mixed with $30 \mathrm{~S}$ complexes, a $20 \%$ increase in fluorescence $\left(3 \mathrm{~s}^{-1}\right)$ was observed (Fig. 21A). The chase of $\mathrm{IF}_{1}(\mathrm{Al} \times 555)$ from the complex with a 20 -fold excess of non-fluorescent factor resulted in a corresponding 15-20\% fluorescence decrease

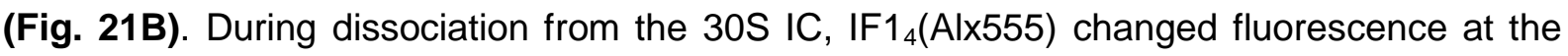
rate of $0.025 \mathrm{~s}^{-1}$ (predominant phase), when IF3 was present on the $30 \mathrm{~S}$ complex. In the absence of IF3, IF1 dissociation was 7-fold faster $\left(0.18 \mathrm{~s}^{-1}\right)$ in agreement with previous reports that IF1 and IF3 stabilize each other on the 30S complex (Milon et al., 2012; Zucker and Hershey, 1986). 

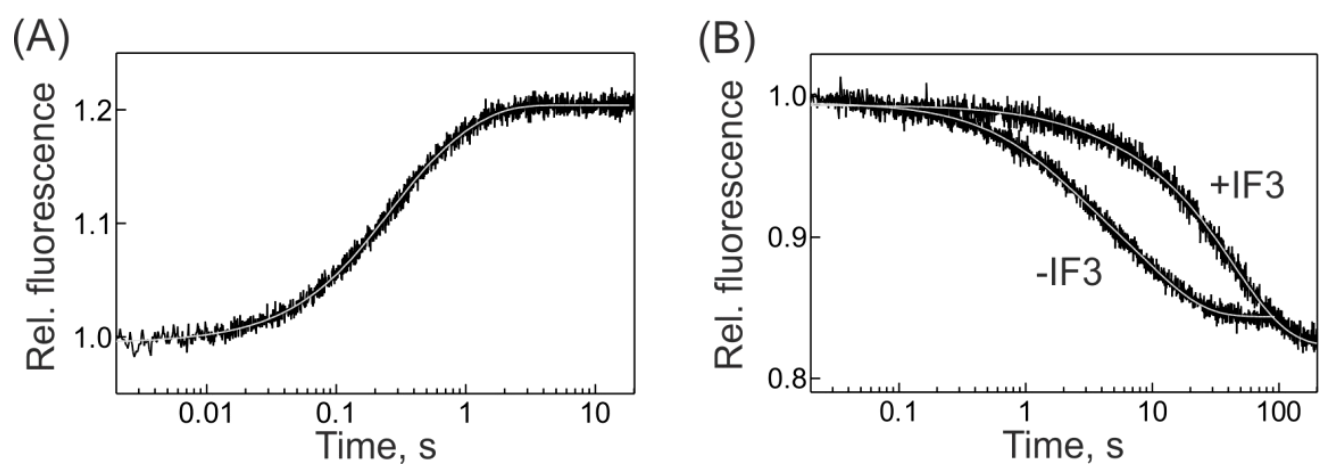

Figure 21. Binding and dissociation kinetics of IF $1_{4}(A \mid x 555)$ on the 30 S IC. Reactions were performed by rapidly mixing indicated components in a stopped-flow apparatus and following the fluorescence changes of IF1 ${ }_{4}(\mathrm{Al} \times 555)$. (A) Time courses $\mathrm{IF} 1_{4}(\mathrm{Al} \times 555)$ binding to 30S PIC (lacking IF1). (B) Time courses of IF1 ${ }_{4}(\mathrm{Al} \times 555)$ dissociation from the $30 \mathrm{~S} \mathrm{IC}$, formed in the presence or absence of IF3, upon chase with 20-fold excess of non-fluorescent IF1.

Change in the environment of IF1 after subunit association was followed by monitoring fluorescence changes of $\mathrm{IF}_{4}(\mathrm{Al} \times 555)$ (Fig. 22A), upon adding increasing concentrations of 50 S subunits to 30 S IC (Fig. 22B). After 50 S subunit joining, a biphasic decrease in fluorescence was observed, with the first major phase ( $80 \%$ of the total amplitude change) occurring with the rate of $4.7 \mathrm{~s}^{-1}$. The apparent rate of the reaction was 2.5 fold faster in the absence of IF3 (Fig. 22C; Table 1) and may be attributed to the faster 50 S subunit joining to 30S IC. When GTP was replaced by GTPYS, the first phase of fluorescence change remained unaffected while the second minor phase, which represents IF1 dissociation (see next section) was abolished (Fig. 22D). 
(A)

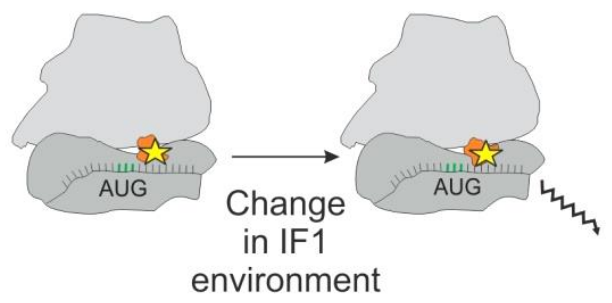

(C)

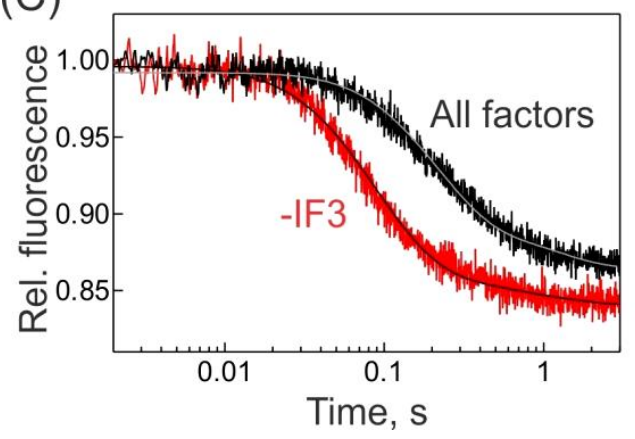

(B)

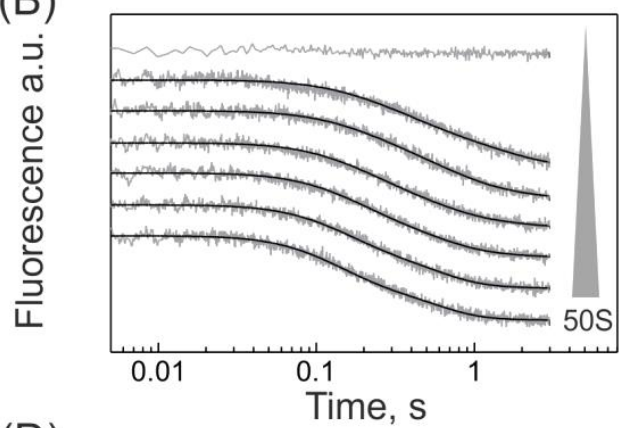

(D)

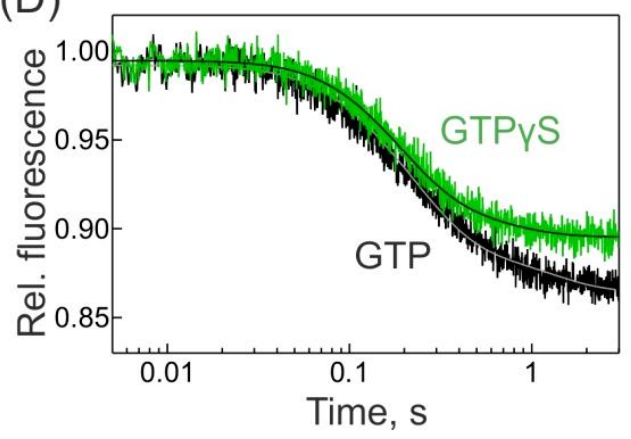

Figure 22. Change in IF1 environment during 70S IC formation. 30S IC $(0.05 \mu \mathrm{M})$ formed with $\mathrm{IF} 1_{4}(\mathrm{Al} \times 555)$, was rapidly mixed with $50 \mathrm{~S}$ subunits in a stopped-flow apparatus and the decrease in $\mathrm{IF}_{4}(\mathrm{Al} \times 555)$ fluorescence was used to monitor the change in IF1 environment on the 70S IC. (A) Schematic of IF1 environment change on the 70S IC. (B) Time courses of change in environment of $\mathrm{IF}_{4}$ (Alx555) upon mixing $30 \mathrm{~S} \mathrm{IC}$ with increasing concentrations of $50 \mathrm{~S}$ subunits $(0.15-1 \mu \mathrm{M})$. Control measurements were performed in the absence of $50 \mathrm{~S}$ subunits. (C) Time courses of change in environment of $\mathrm{IF}_{4}(\mathrm{Alx} 555)$ upon mixing $30 \mathrm{~S} \mathrm{IC}$ formed in the presence of all factors or absence of IF3 with 50 S subunits $(1 \mu \mathrm{M})$. (D) Time courses of change in environment of $\mathrm{IF}_{4}(\mathrm{Al} \times 555)$ upon mixing $30 \mathrm{~S} \mathrm{IC}$, formed in the presence of GTP or GTPYS, with 50 S subunits $(1 \mu \mathrm{M})$.

\subsubsection{Dissociation of IF1 from the 70 S complex}

The direct interaction of IF1 with the ribosome was monitored via FRET between $\mathrm{IF}_{4}(\mathrm{Att0} 540 \mathrm{Q})$ and $30 \mathrm{~S}_{\mathrm{S} 13}(\mathrm{Al} \times 488)$. The binding of quencher IF1 ${ }_{4}(\mathrm{Att0} 540 \mathrm{Q})$ to $30 \mathrm{~S}_{\mathrm{S} 13}(\mathrm{Alx} 488)$ resulted in a $20 \%$ decrease of the fluorescence which occurred with the rate of $42 \mathrm{~s}^{-1}$ (Fig. 23A). The chase of $\mathrm{IF}_{1}$ (Atto540Q) from the $30 \mathrm{~S}_{\mathrm{S13}}$ (Alx488) IC, with a 20fold excess of non-fluorescent factor, led to a corresponding $~ 20 \%$ increase of fluorescence, indicative of IF1 dissociation from the 30S IC which took place with the rates of $0.01 \mathrm{~s}^{-1}$ and $0.08 \mathrm{~s}^{-1}$ from complexes formed in the presence or absence of IF3, respectively (Fig. 23B). 

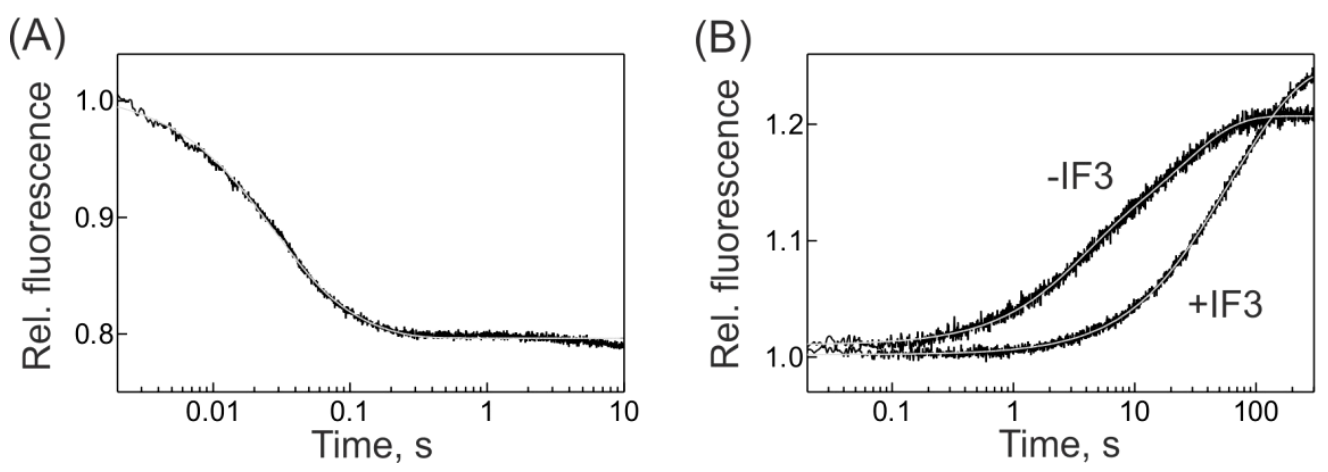

Figure 23. Binding and dissociation kinetics of $\mathrm{IF}_{4}(\mathrm{Atto540Q})$ on the ${ }_{30 \mathrm{~S}_{\mathrm{S} 13}}(\mathrm{AlX488)}$ IC. Reactions were performed by rapidly mixing indicated components in a stopped-flow apparatus and following the FRET between $30 \mathrm{~S}_{\mathrm{S13}}(\mathrm{Al} \times 488) \mathrm{IC}$ and IF1 ${ }_{4}$ (Atto540Q). (A) Time courses $\mathrm{IF}_{4}\left(\right.$ Atto540Q) binding to $30 \mathrm{~S}_{\mathrm{S13}}(\mathrm{Alx} 488)$ PIC (lacking IF1). (B) Time courses of $\mathrm{IF}_{4}\left(\right.$ Atto540Q) dissociation from the $30 \mathrm{~S}_{\mathrm{S13}}(\mathrm{Al} \times 488) \mathrm{IC}$, formed in the presence or absence of IF3, upon chase with 20 -fold excess of non-fluorescent IF1.

Differences were observed between the binding and dissociation kinetics of IF1 on the $30 \mathrm{~S}$ complex monitored using the two IF1 reporters. When IF1 $1_{4}$ (Atto540Q) was mixed with $30 \mathrm{~S}_{\mathrm{S}_{13}}$ (Alx488) PIC, the signal change was 10 -fold faster than that observed upon $\mathrm{IF} 1_{4}(\mathrm{Al} \times 555)$ interaction with $30 \mathrm{~S}$ PIC (Fig. 21A), indicating that IF1 binding (depicted by the former reporter) is followed by rearrangements of the complex (depicted by the latter observable). Similarly, the rate of $\mathrm{IF}_{4}\left(\mathrm{Att0} 540 \mathrm{Q}\right.$ ) chase from the $30 \mathrm{~S}_{\mathrm{S} 13}(\mathrm{Al} \times 488) \mathrm{IC}$ was 2fold slower than the rate of $\mathrm{IF}_{4}(\mathrm{Alx} 555)$ chase from $30 \mathrm{~S} \mathrm{PIC} \mathrm{(Fig.} \mathrm{21B),} \mathrm{indicating} \mathrm{that} \mathrm{IF1}$ dissociation is preceded by a reversal of rearrangements of the $30 \mathrm{~S}$ complex.

IF1 dissociation from the 70S complex was monitored by loss of FRET between $\mathrm{IF}_{1}(\mathrm{Att0} 540 \mathrm{Q})$ and $70 \mathrm{~S}_{\mathrm{S} 13}(\mathrm{Alx} 488)$ IC (Fig. 24A). When increasing concentrations of $50 \mathrm{~S}$ subunits were added to $30 \mathrm{~S} \mathrm{IC} \mathrm{containing} \mathrm{the} \mathrm{FRET} \mathrm{couple,} \mathrm{an} \mathrm{increase} \mathrm{in} \mathrm{fluorescence} \mathrm{was}$ observed (Fig. 24B), indicating that IF1 moves away from the 30S subunit during 70 S IC formation. The first, minor phase ( $30 \%$ of the total amplitude) occurred independently of GTP hydrolysis (Fig. 24C) and was attributed to the change in IF1 environment which precedes dissociation of the factor. The second, major phase ( $50 \%$ of the total amplitude) representing IF1 release was abolished when GTPYS replaced GTP in the system. 50S joining and GTP hydrolysis by IF2 dramatically stimulated IF1 dissociation from the ribosome, as seen by a 200-fold increase in the rate of IF1 release from the $70 \mathrm{~S}$ complex, as compared with the 30S IC (apparent rates of $2 \mathrm{~s}^{-1}$ (Table 1) and $0.01 \mathrm{~s}^{-1}$ (Fig. 23B), respectively). 
(A)

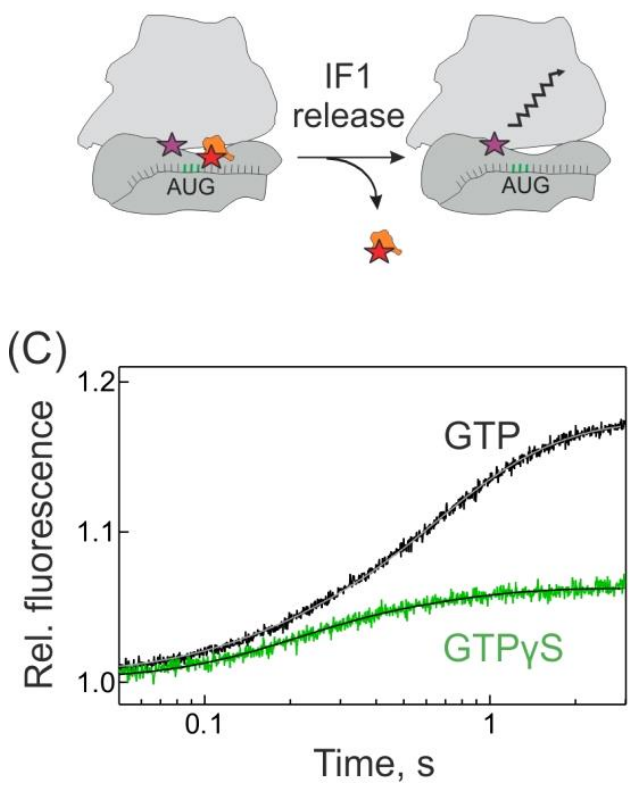

(B)

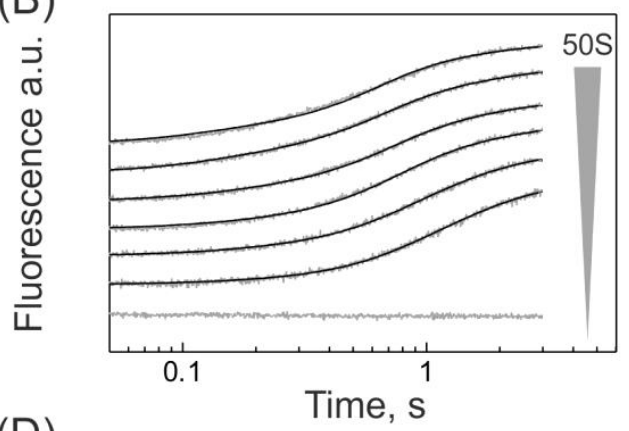

(D)

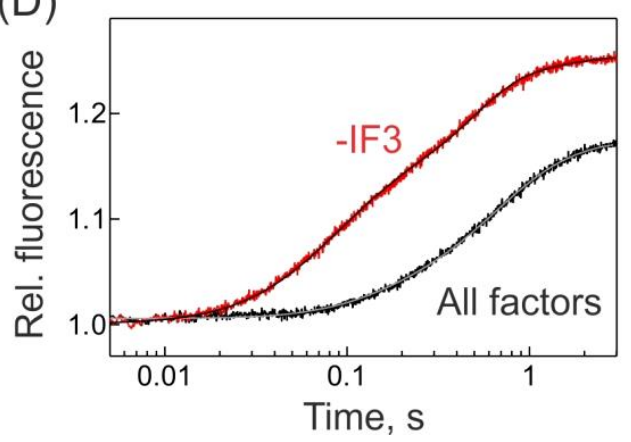

Figure 24. Dissociation of IF1 during 70 S IC formation. 30S IC $(0.05 \mu \mathrm{M})$ formed with $30 \mathrm{~S}_{\mathrm{S13}}$ (Alx488) and $\mathrm{IF}_{4}($ Atto540Q), was rapidly mixed with $50 \mathrm{~S}$ subunits in a stopped-flow apparatus and the loss of FRET (which resulted in fluorescence increase) was used to monitor IF1 dissociation from the $70 \mathrm{SIC}$. (A) Schematic of $\mathrm{IF} 1_{4}$ (Atto540Q) dissociation from the $70 S_{S_{13}}($ Alx488) IC. (B) Time courses of IF1 dissociation from the 70S IC upon mixing $30 \mathrm{~S}$ IC with increasing concentrations of $50 \mathrm{~S}$ subunits $(0.15 \mu \mathrm{M}-1 \mu \mathrm{M})$. Control measurements were performed in the absence of $50 \mathrm{~S}$ subunits. (C) Time courses of IF1 dissociation from the 70S IC upon mixing 30S IC, formed in the presence of GTP or GTPYS, with 50S subunits $(1 \mu M)$. (D) Time courses of IF1 dissociation from the 70 S IC upon mixing 30 S IC, formed in the presence of all factors or absence of IF3 with 50 S subunits $(1 \mu \mathrm{M})$.

In the presence of IF3, the extent of fluorescence change upon IF1 release from the 70S complex was slightly less than when IF1 was chased from the 30S IC (Fig. 23B), indicating that only $\sim 80 \%$ of the bound IF1 dissociates from the 70 complex. A third slow phase ( $20 \%$ of the total amplitude change, rate $-0.1 \mathrm{~s}^{-1}$ ), resulted from slow $50 \mathrm{~S}$ subunit joining to incorrectly-formed 30S IC and accounted for the remaining amplitude change. In the absence of IF3, 50S rapidly associates with all $30 \mathrm{~S}$ complexes, regardless of their conformation. Hence, the slow phase was not observed (Fig. 24D) and the entire amplitude change was equally shared between the first two phases. The first phase depicting the change of IF1 environment appeared faster when IF3 was lacking from the complex, while the rate of IF1 dissociation was not affected (Table 1).

The dependence of IF1 release from the 70S IC on GTP hydrolysis by IF2 was a surprising finding. To understand the significance of this interplay, efforts were undertaken to uncouple 
the two events. IF3 has been shown to stabilize IF1 on the $30 \mathrm{~S}$ complex. Thus, it is possible that in the absence of IF3, IF1 may be destabilized enough to dissociate from the $70 S$ complex even in the absence of GTP hydrolysis. 30S IC, containing GTPYS but lacking IF3, was mixed with $50 \mathrm{~S}$ subunits and IF1 dissociation was monitored with time. The absence of IF3 did not allow IF1 release from the 70S complex to occur when GTP hydrolysis was inhibited (Fig. 25A), indicating that the interplay was specific to IF1 and IF2.
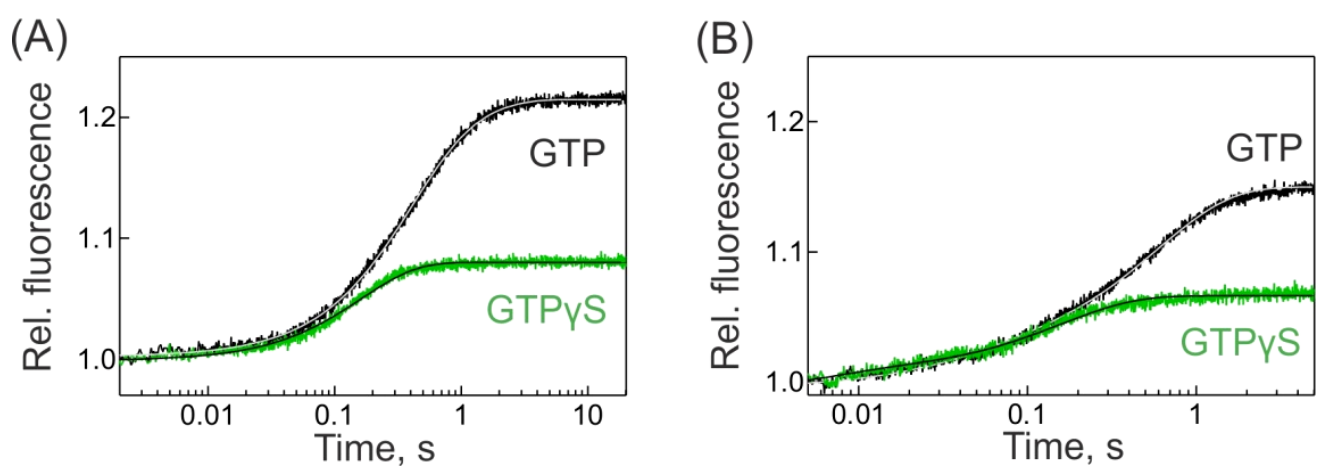

Figure 25. Efforts to uncouple IF1 dissociation from GTP hydrolysis. 30S IC $(0.05 \mu \mathrm{M})$ formed with $30 \mathrm{~S}_{\mathrm{S} 13}(\mathrm{Al} \times 488)$ and $\mathrm{IF}_{4}(\mathrm{Atto540Q})$, in the presence of GTP or GTPyS, was rapidly mixed with $50 \mathrm{~S}$ subunits $(0.25 \mu \mathrm{M})$ in a stopped-flow apparatus and the loss of FRET (which resulted in fluorescence increase) was used to monitor IF1 dissociation from the 70S IC. (A) Time courses of IF1 dissociation from the 70S IC upon mixing 30S IC (lacking IF3) with 50 S subunits. (B) Time courses of IF1 dissociation from 70S IC upon mixing 30 S IC (formed with IF $2 \Delta \mathrm{N}$ mutant) with $50 \mathrm{~S}$ subunits.

On the 30S IC, IF1 contacts the NTD of the $\alpha$-form (i.e. which contains the full-length Ndomain) of E. coli IF2 (Julian et al., 2011). Hence, we checked whether IF1 release can be uncoupled from GTP hydrolysis by using a truncated version of IF2 lacking the entire NTD (IF2 $\Delta \mathrm{N}$ (Moreno et al., 1999)). In the absence of GTP hydrolysis by IF2 $\Delta \mathrm{N}, \mathrm{IF} 1_{4}($ Atto540Q) was not released from the $70 \mathrm{~S}_{\mathrm{S} 13}(\mathrm{Alx} 488$ ) complex (Fig. 25B), indicating that IF1 release from the $70 S$ complex is not mediated by the loss of interaction with the NTD of IF2. Thus, it is possible that (i) IF1 interacts with a different domain of IF2 on the 70S IC, or (ii) the release of IF1 may be induced by conformational changes of the ribosome triggered by GTP hydrolysis.

Based on the positioning of IF1 at the A site of the ribosome, it was suggested that IF1 may be involved in checking the fidelity of start codon-anticodon interaction (Antoun et al., 2006a; Milon et al., 2008; Qin et al., 2012). The correct base-pairing and accommodation of fMettRNA $^{\text {fMet }}$ in the $\mathrm{P}$ site after GTP hydrolysis could, in turn, trigger the dissociation of IF1 from the $70 \mathrm{~S}$ complex. The importance of the identity of the mRNA start codon was checked by 
preparing 30S IC with an mRNA containing an AUG or AUU start codon (Fig. 26A). Although the extent of IF1 release was not affected by replacing AUG with an AUU start codon, the rate of the reaction decreased by 100 -fold. This is because, in the presence of IF3, 50 S joining to $30 \mathrm{~S}$ complexes containing a non-cognate start codon is very slow, and becomes rate-limiting for subsequent events (Grigoriadou et al., 2007b; Milon et al., 2008). Hence, the complexes were formed in the absence of IF3 to promote rapid subunit joining, and the effect of AUU and UUG start codon on IF1 release was tested (Fig. 26B-C). The identity of the start codon did not affect the extent or rate of IF1 release, suggesting that correct codonanticodon base pairing is not a pre-requisite for, and does not trigger, IF1 dissociation from the 70S complex. Despite several efforts, the reason for the dependence of IF1 release on GTP hydrolysis by IF2 remains unknown and clearly merits further investigation.
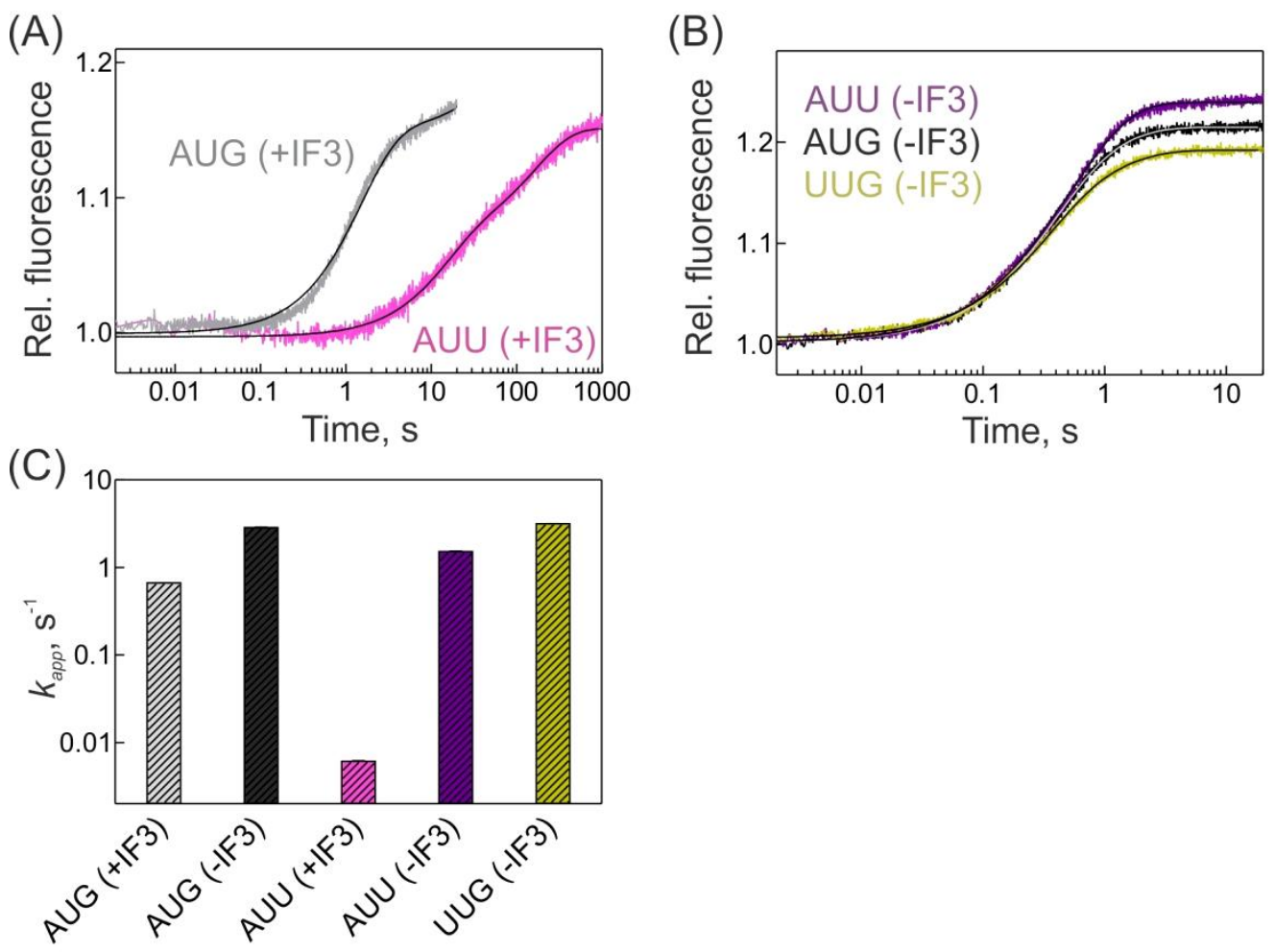

Figure 26. Effect of non-cognate start codons on IF1 dissociation. 30S IC $(0.05 \mu \mathrm{M})$ formed with $30 \mathrm{~S}_{\mathrm{S}_{13}}(\mathrm{Al} \times 488)$ and $\mathrm{IF}_{4}(\mathrm{Atto540Q})$, in the presence of mRNA containing different start codon sequences, was rapidly mixed with 50 S subunits $(0.25 \mu \mathrm{M})$ in a stoppedflow apparatus and the loss of FRET (which resulted in fluorescence increase) was used to monitor IF1 dissociation from the 70S IC. (A) Time courses of IF1 dissociation from the 70S IC upon mixing $30 \mathrm{~S} \mathrm{IC} \mathrm{(containing} \mathrm{all} \mathrm{factors)} \mathrm{with} 50 \mathrm{~S}$ subunits. (B) Time courses of IF1 dissociation from 70S IC upon mixing 30S IC (lacking IF3) with 50S subunits. (C) Apparent rates of the predominant phase derived from double-exponential fitting of time courses in (A)(B); error bars represent standard error of the fit. 


\subsection{Dynamics of IF3 on the 70S IC}

The interaction of IF3 on the ribosome was monitored by FRET between IF3 ${ }_{166}(\mathrm{Al} \times 488)$ and IF2 757 (Atto540Q). Binding of IF3 ${ }_{166}$ (Alx488) to 30 S IC containing IF2 257 (Atto540Q) resulted in a 20\% decrease in fluorescence (Fig. 27A). IF3 $(0.1 \mu \mathrm{M})$ associated very rapidly with the $30 \mathrm{~S}$ complex $\left(100 \mathrm{~s}^{-1}\right)$. The chase of $\mathrm{IF}_{166}$ (Alexa488) from the $30 \mathrm{~S}-\mathrm{IF}_{757}$ (Atto540Q) complex, with a 20-fold excess of non-fluorescent IF3, resulted in a corresponding increase of fluorescence by $20 \%$ (Fig. 27B). In the presence of IF1, IF3 dissociated from the 30 S IC with a rate of $7 \mathrm{~s}^{-1}$ while, in the absence of IF1, IF3 dissociation was 8-fold faster $\left(54 \mathrm{~s}^{-1}\right)$. These results are in agreement with previous studies which suggested that IF1 stabilizes the interaction of IF3 with the 30S complex (Antoun et al., 2006b; Milon et al., 2012).
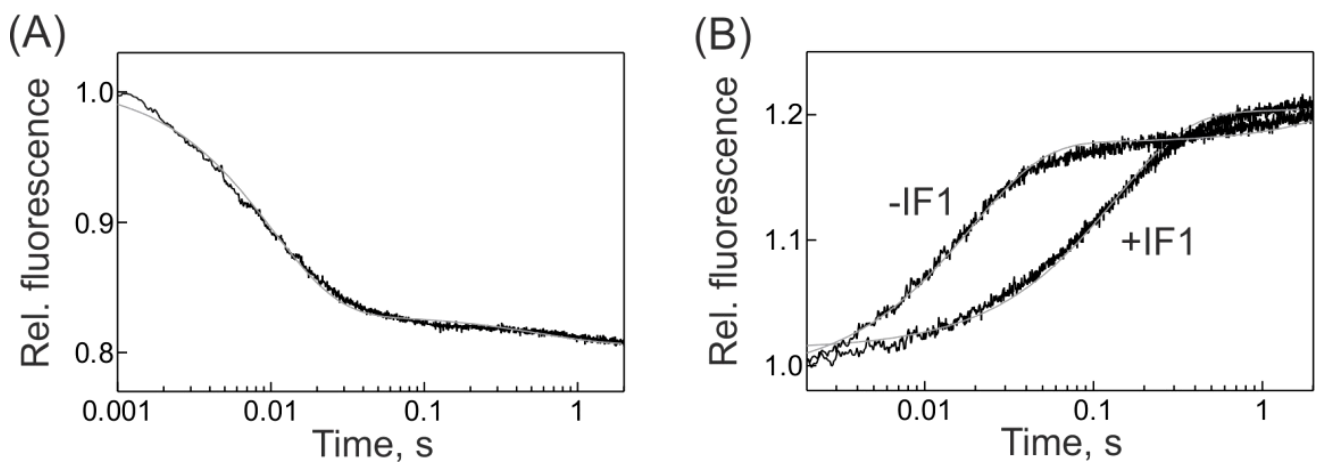

Figure 27. Binding and dissociation kinetics of IF $3_{166}(A \mid x 488)$ on the 30 S IC containing IF2 ${ }_{757}$ (Atto540Q) IC. Reactions were performed by rapidly mixing indicated components in a stopped-flow apparatus and following the changes in FRET between IF3 ${ }_{166}(\mathrm{Alx} 488)$ and IF2 757 (Atto540Q). (A) Time courses IF3 ${ }_{166}$ (Alx488) binding to 30S - IF2 ${ }_{757}$ (Atto540Q) PIC (lacking IF3). (B) Time courses of IF3 ${ }_{166}$ (Alx488) dissociation from the $30 \mathrm{~S}-\mathrm{IF} 2_{757}$ (Atto540Q) IC, formed in the presence or absence of IF1, upon chase with 20-fold excess of nonfluorescent IF3.

Dissociation of IF3 from the 70S complex was monitored via the loss of FRET between IF3 ${ }_{166}$ (Alx488) and IF2 $2_{757}$ (Atto540Q) after 50S subunit joining (Fig. 28A). Upon 50 S subunit joining to 30 S IC containing the IF2-IF3 FRET couple, an increase in fluorescence was observed indicating that 50 S subunit association causes IF3 to move away from IF2 on the ribosome (Fig. 28B). The extent of fluorescence increase was $20 \%$, similar to that observed previously during the chase of IF3 from 30 S IC (Fig. 27B), suggesting that the signal change might reflect the dissociation of IF3 from the ribosome. 
(A)

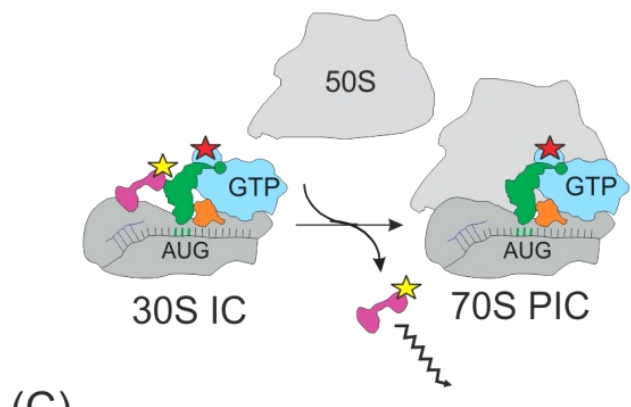

(C)

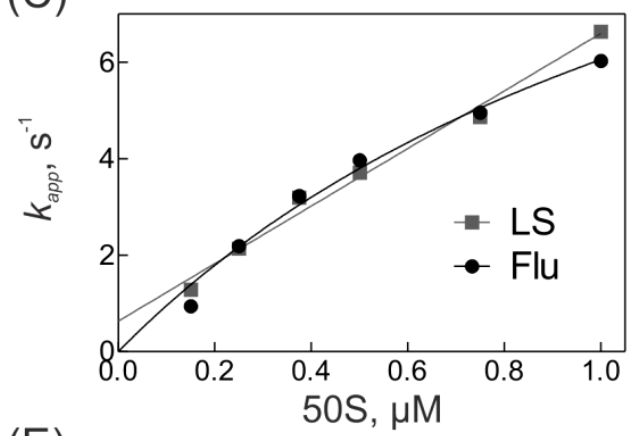

(E)

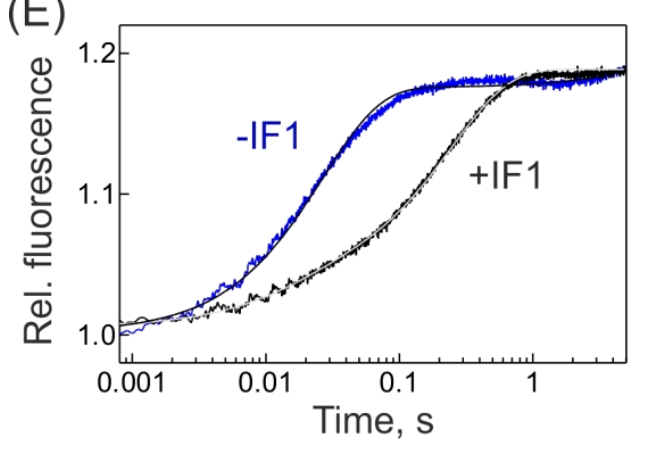

(B)

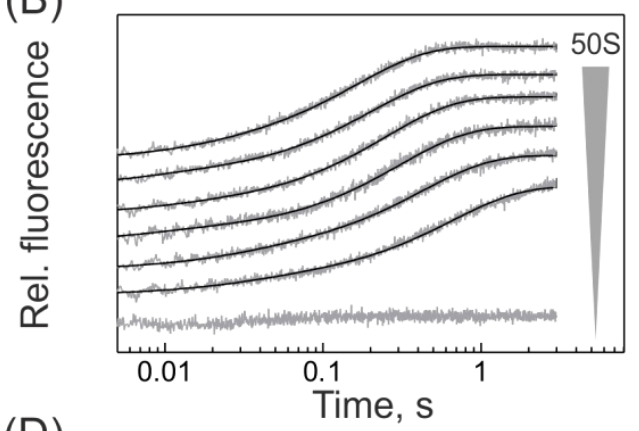

(D)
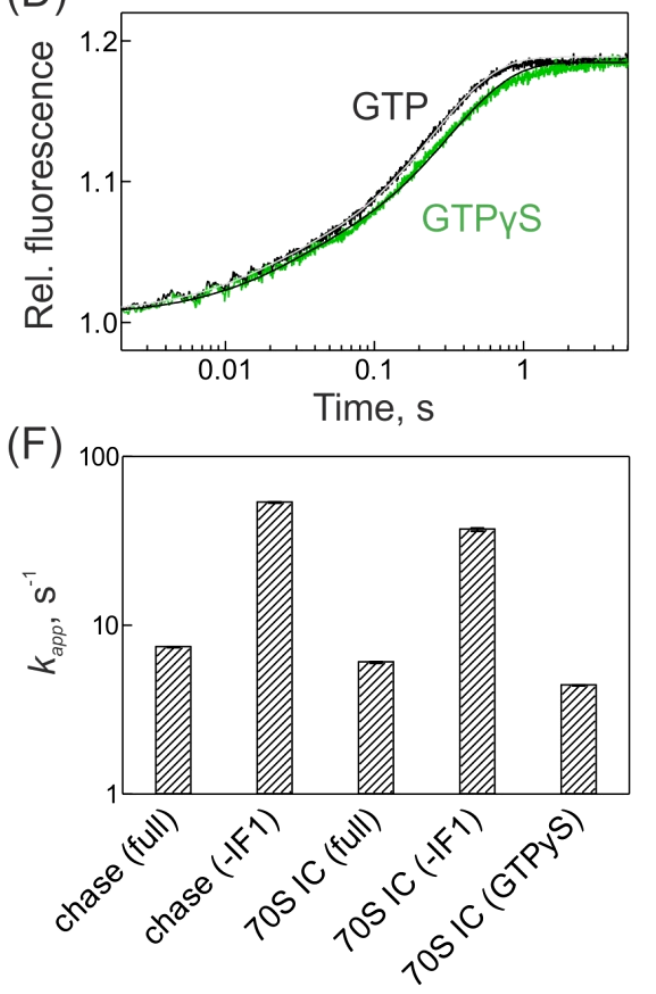

Figure 28. Dissociation of IF3 during 70 S IC formation. 30 S IC $(0.05 \mu \mathrm{M})$, formed with IF ${ }_{166}$ (Alx488) and IF2 757 (Atto540Q), was rapidly mixed with 50 S subunits in a stopped-flow apparatus and the loss of FRET between IF3 ${ }_{166}$ (Alx488) IC and IF2 $2_{757}$ (Atto540Q) (which resulted in a fluorescence increase) was used to monitor IF3 dissociation from the 70 S IC. (A) Schematic of IF3 ${ }_{166}$ (Alx488) dissociation from the 70 S IC containing IF2 ${ }_{757}$ (Atto540Q) (B) Time courses of IF3 dissociation from the 70S IC upon mixing 30S IC with increasing concentrations of $50 \mathrm{~S}$ subunits $(0.15-1 \mu \mathrm{M})$. Control measurements were performed in the absence of $50 \mathrm{~S}$ subunits. (C) Dependence of apparent rates of subunit joining (monitored by LS in the presence of the IF3-IF2 FRET couple) and IF3 dissociation from the 70S IC (Flu) on $50 \mathrm{~S}$ concentration. Rates and standard errors (error bars smaller than symbol size are not visible) were derived from double-exponential fitting of time courses. (D) Time courses of IF3 dissociation from 70S IC upon mixing 30S IC, formed in the presence of GTP or GTPYS, with 50 S subunits $(1 \mu \mathrm{M})$. (E) Time courses of IF3 dissociation from the $70 \mathrm{~S} \mathrm{IC} \mathrm{upon} \mathrm{mixing} 30 \mathrm{~S}$ IC, formed in the presence of all factors or absence of IF1 with 50 S subunits $(1 \mu \mathrm{M})$. (F) Apparent rate constants of the predominant phase derived from double-exponential fitting of time courses in (D)-(E) and Fig. 27B; error bars represent standard error of the fit. 
The dependence of the apparent rate of the reaction on increasing concentrations of $50 \mathrm{~S}$ subunits almost overlapped with the rates of subunit joining to 30 S IC formed with the IF2IF3 FRET couple (Fig. 28C). This suggested that IF3 is released from the $70 \mathrm{~S}$ complex immediately upon subunit association. Comparison of time courses obtained in the presence of GTP and GTPYS showed that the reaction was entirely independent of IF2-dependent GTP hydrolysis (Fig. 28D). IF3 dissociation from the 70S complex was 6- fold faster in the absence of IF1 $\left(37 \mathrm{~s}^{-1}\right)$ than in its presence $\left(6 \mathrm{~s}^{-1}\right)$ (Fig. 28E). Interestingly, the rates of IF3 release from the $30 \mathrm{~S}$ and $70 \mathrm{~S}$ complex, in the presence or absence of IF1, were very similar (Fig. 28F). These results indicate that $50 \mathrm{~S}$ subunit joining does not accelerate the dissociation of IF3 from the ribosome, suggesting that the propensity for IF3 to dissociate is determined by the conformation of the $30 \mathrm{~S} \mathrm{IC}$, which in turn depends upon the TIR of the mRNA, codon-anticodon recognition and the proper arrangement of all initiation components on the $30 \mathrm{~S}$ subunit (Milon et al., 2008). 


\subsection{Formation of the $1^{\text {st }}$ peptide bond.}

To study the transition of the $30 \mathrm{~S} \mathrm{IC} \mathrm{to} \mathrm{an} \mathrm{elongation-competent} 70 \mathrm{~S} \mathrm{IC}$, the first peptide bond formation was measured (Fig. 29A) using the quench-flow apparatus (Antoun et al., 2003; Milon et al., 2008; Tomsic et al., 2000). 30S IC was rapidly mixed with increasing concentrations of $50 \mathrm{~S}$ subunits and EF-Tu-GTP-Phe-tRNA ${ }^{\text {Phe }}$ ternary complex (TC) (0.4 $\mu \mathrm{M}$ ), and the formation of fMet-Phe dipeptide was followed with time (Fig. 29B).

(A)

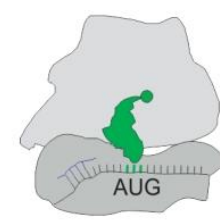

70 S IC

(C)
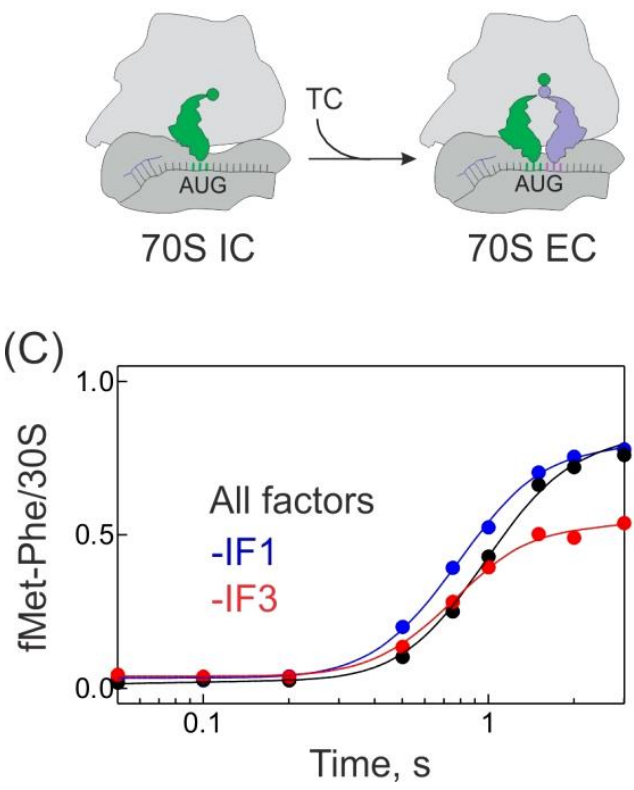

(B)

(D)
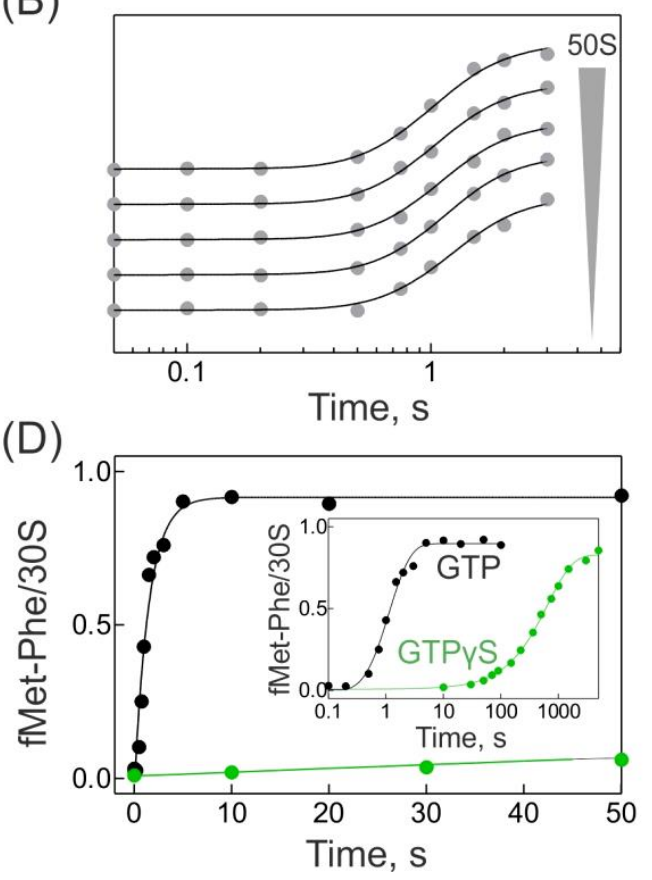

Figure 29. Formation of fMet-Phe dipeptide. 30S IC $(0.1 \mu \mathrm{M})$ was rapidly mixed with $50 \mathrm{~S}$ subunits and EF-Tu-GTP-Phe-tRNA ${ }^{\text {Phe }}$ ternary complex (TC) $(0.4 \mu \mathrm{M})$ in a quench-flow apparatus and the formation of fMet-Phe was measured with time. (A) Schematic of the first peptide bond formation. (B) Time courses of fMet-Phe formation upon mixing 30S IC with increasing concentrations of $50 \mathrm{~S}$ subunits $(0.3-1 \mu \mathrm{M})$ and EF-Tu TC. (C) Time courses of fMet-Phe formation upon mixing $30 \mathrm{~S} \mathrm{IC}$, formed in the presence of all factors and absence of IF1 or IF3 with 50S subunits $(1 \mu \mathrm{M})$ and EF-Tu TC. (D) Time courses of fMet-Phe dipeptide formation upon mixing 30S IC, formed in the presence of GTP or GTPYS, with 50S subunits $(1 \mu \mathrm{M})$ and EF-Tu TC (inset-extended time course).

Peptide bond formation occurred after a $300 \mathrm{~ms}$ lag phase and the lack of IF1 or IF3 did not affect the timing of the reaction (Fig. 29C). In agreement with previous reports, the extent of fMet-Phe formation was decreased by $40 \%$ in the absence of IF3, suggesting that under these conditions, a certain portion of the $70 \mathrm{~S}$ complexes formed lacked fMet-tRNA ${ }^{\text {iMet }}$ (Antoun et al., 2006b). The replacement of GTP with GTPYS inhibited the reaction (Fig. 
29D), indicating that GTP hydrolysis by IF2 is essential for the productive transition of $70 S$ complexes into an elongation-ready state. Assuming that $70 \mathrm{~S}$ maturation into the $70 \mathrm{~S} \mathrm{EC}$ is limited by GTPYS hydrolysis and subsequent IF2 dissociation, dipeptide formation can be used as an indicator to provide an estimate for the rate of hydrolysis of GTPYS by IF2 $\left(0.0015 \mathrm{~s}^{-1}\right)$ (Fig. 29D (inset)). 


\subsection{Global fitting of datasets}

As mentioned previously, the apparent rate $\left(\mathrm{k}_{\mathrm{app}}\right)$ of a reaction can be derived from the exponential fitting of only those time courses which do not contain a delay phase before the start of the reaction. The $k_{\text {app }}$ of a binding reaction (such as subunit joining) is the resultant of its elemental association and dissociation rates, while the $k_{\text {app }}$ of a monomolecular reaction may be influenced by the timing of preceding steps. Hence, the elemental rate constants describing the individual forward and backward directions of each reaction were calculated by global fitting. Time courses, obtained for each reaction at increasing $50 \mathrm{~S}$ subunit concentrations, were collectively evaluated using the numerical integration software, KinTec explorer to derive the elemental rate constants of each step $\left(k_{1}-k_{10}\right)$. By comparing apparent rate constants, delay phase of time courses and dependence of different reactions on GTP hydrolysis, a 10-step model was formulated to evaluate the time courses of reactions:

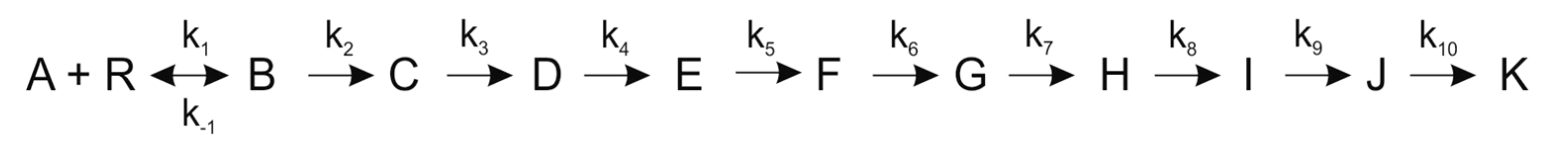

where $A$ and $R$ refer to the $30 S I C$ and 50 S subunit, respectively, and $B$ to $K$ are intermediates on the pathway from the 30S IC to 70S EC formation. The kinetics of subunit joining are described by the elemental rates constants $k_{1}$ and $k_{-1}$. GTPase activation corresponds to the $\mathrm{B} \rightarrow \mathrm{C}$ transition (rate constant $\mathrm{k}_{2}$ ) because - based on the exponential fitting - it is the fastest rearrangement step which is independent of GTP hydrolysis. The transition $\mathrm{C} \rightarrow \mathrm{D}\left(\mathrm{k}_{3}\right)$ is assigned to the change of IF1 environment which also does not require GTP hydrolysis to take place. All subsequent steps of the reaction pathway are inhibited in the absence of GTP hydrolysis. The sequence of the following steps is assigned on the basis of their kinetics, in particular by the characteristic duration of the delay phase. Single-turnover $\mathrm{Pi}$ release corresponds to the step $\mathrm{D} \rightarrow \mathrm{E}\left(\mathrm{k}_{4}\right)$. The subsequent rounds of multiple-turnover GTP hydrolysis and Pi release, uncoupled from initiation, are described by a late, very slow step $\mathrm{J} \rightarrow \mathrm{K}\left(\mathrm{k}_{10}\right)$. tRNA ${ }^{\text {iMet }}$ release from IF2 is given by $\mathrm{E} \rightarrow \mathrm{F}\left(\mathrm{k}_{5}\right)$. Three reactions occur around the same time and are described by a single step $F \rightarrow G\left(k_{6}\right)-I F 2$ release from the $70 \mathrm{~S}$ complex, IF1 release from the 70S complex and GDP release from IF2. Binding of IF2 to the $30 \mathrm{~S}$ complex during the second round of initiation is depicted by the transition $\mathrm{G} \rightarrow \mathrm{H}\left(\mathrm{k}_{7}\right)$. The final step of fMet-Phe formation $(\mathrm{H} \rightarrow \mathrm{l})$ corresponds to $\mathrm{k}_{8}$.

An additional step described by $\mathrm{I} \rightarrow \mathrm{J}\left(\mathrm{k}_{9}\right)$ was included in the model to account for the minor phase observed at late time points of the reactions. This step can be explained by slow $50 \mathrm{~S}$ joining to incorrectly formed or "inactive" 30S IC (McGinnis et al., 2015), and is observed 
predominantly in the presence of IF3 which confers an anti-association conformation on these complexes. Because the kinetics of subunit joining was slower in the presence of the FRET pair, IF3 ${ }_{166}(\mathrm{Al} \times 488)$ and IF2 ${ }_{757}$ (Alx488), as compared to their unlabeled counterparts (Fig. 10C-D), the dataset for IF3 dissociation could not be incorporated into the 10-step kinetic model to derive elemental rates of the reaction.

All reactions occurring after subunit joining were assumed to be quasi-irreversible, which is very likely for the release reactions (of Pi, IF1, IF2, GDP), and represents a simplification of the model, justified by the commitment of $70 S$ complex in the forward pathway towards maturation, and the absence of any evidence for the existence of highly reversible steps. To facilitate the fitting of such large datasets, the rates of the slow steps in the model were assigned fixed values $\left(0.5 \mathrm{~s}^{-1}\right.$ for $\mathrm{k}_{9} ; 0.01 \mathrm{~s}^{-1}$ for $\left.\mathrm{k}_{10}\right)$. Fitting data with alternative models, i.e. with a larger number of steps or different order of events, was not successful.

In the absence of IF1 or IF3, several reactions (such as subunit joining, GTPase activation, change in IF1 environment, Pi release and tRNA ${ }^{\text {fMet }}$ release from IF2) appeared faster. To understand if the increased apparent rate was a result of faster $50 \mathrm{~S}$ docking or an intrinsic property of the reaction, the elemental rate constant of each event was calculated. For complexes lacking IF1 or IF3, LS was monitored at varying 50 S concentrations. For each of the remaining reaction observables, single time courses were obtained at a fixed concentration of $50 \mathrm{~S}$ subunits $(1 \mu \mathrm{M})$. The time courses were fitted by numerical integration using the 10-step model described above, with the following modifications- (i) IF1-dependent steps were removed from the reaction scheme when IF1 was absent, (ii) the GTPase activation step, which in the full system is a kinetically discrete reaction, in the absence of IF3 becomes indistinguishable from the 50S subunit docking; hence, the two steps were grouped, and (iii) IF2 (and IF1) dissociation from 70S complex lacking IF1 or IF3 occurred around the same time as the release of $\mathrm{TRNA}^{\mathrm{fMet}}$ from IF2; hence, the three steps were grouped.

Because there was no difference between the rates of subunit joining in the presence of GTP or GTPYS (Fig. 12B), the time courses of LS (GTP) measured at increasing 50 S subunit concentrations were modeled with singular time courses, obtained with the remaining observables, in the presence of GTPYS and at a fixed 50 S subunit concentration $(1 \mu \mathrm{M})$. Time courses for the observables which displayed a signal change in the presence of GTPYS were fitted with a 4-step model $\left(\mathrm{A} \rightarrow \mathrm{B}(\mathrm{LS}) ; \mathrm{B} \rightarrow \mathrm{C}\right.$ (mant-GTPYS); $\mathrm{C} \rightarrow \mathrm{D}\left(\mathrm{IF}_{4}(\mathrm{Al} \times 555)\right)$ and $D \rightarrow E$ (slow subunit joining)). 
The elemental rate constant of each reaction (Table 2), and the overall statistics of the global fitting (Fig. 30) are shown.

Table 2. Summary of elemental rate constants of reactions during 70 S IC formation.

\begin{tabular}{|c|c|c|c|c|c|}
\hline Kinetic step & Observable & $\begin{array}{l}\text { All IFs } \\
\text { GTP }\end{array}$ & $\begin{array}{l}\text {-IF1 } \\
\text { GTP }\end{array}$ & $\begin{array}{l}\text {-IF3 } \\
\text { GTP }\end{array}$ & $\begin{array}{l}\text { All IFs } \\
\text { GTPYS }\end{array}$ \\
\hline $\begin{array}{l}\text { 50S association, } \\
k_{1}\left(\mu M^{-1} s^{-1}\right)\end{array}$ & LS & $30 \pm 6$ & $43 \pm 5$ & $50 \pm 5$ & $23 \pm 6$ \\
\hline $\begin{array}{l}\text { 50S dissociation, } \\
\mathrm{k}_{-1}\left(\mathrm{~s}^{-1}\right)\end{array}$ & LS & $31 \pm 15$ & $\sim 0$ & $2.5 \pm 1.0$ & $13 \pm 10$ \\
\hline $\begin{array}{l}\text { GTPase activation, } \\
\mathrm{k}_{2}\left(\mathrm{~s}^{-1}\right)\end{array}$ & Mant-GTP & $36 \pm 6$ & $24 \pm 5$ & as $\mathrm{k}_{1}$ & $37 \pm 10$ \\
\hline $\begin{array}{l}\text { Change of IF1 } \\
\text { environment, } \mathrm{k}_{3}\left(\mathrm{~s}^{-1}\right)\end{array}$ & $\mathrm{IF} 1_{4}(\mathrm{Al} \times 555)$ & $19 \pm 5$ & n.o. & $17 \pm 5$ & $11 \pm 3$ \\
\hline Pi release, $\mathrm{k}_{4}(\mathrm{~s}-1)$ & Pi-PBP(MDCC) & $4.0 \pm 0.5$ & $3.9 \pm 0.5$ & $4.3 \pm 0.5$ & n.o. \\
\hline $\begin{array}{l}\text { fMet-tRNA }^{\text {fMet }} \text { release } \\
\text { from IF2, } \mathrm{k}_{5}\left(\mathrm{~s}^{-1}\right)\end{array}$ & Bpy-Met-tRNA ${ }^{\text {fMet }}$ & $19 \pm 5$ & $13 \pm 3$ & $10 \pm 3$ & n.o. \\
\hline $\begin{array}{l}\text { IF2 dissociation, } \\
\mathrm{k}_{6}\left(\mathrm{~s}^{-1}\right)\end{array}$ & Mant-GTP & $5.5 \pm 0.5$ & as $\mathrm{k}_{5}$ & as $\mathrm{k}_{5}$ & n.o. \\
\hline $\begin{array}{l}\text { IF1 dissociation, } \\
\mathrm{k}_{6}\left(\mathrm{~s}^{-1}\right)\end{array}$ & $\begin{array}{l}\mathrm{IF}_{4}\left(\mathrm{Atto5}_{40 Q}\right)- \\
30 \mathrm{~S}_{\mathrm{S} 13}(\mathrm{Al} \times 488)\end{array}$ & $5.5 \pm 0.5$ & n.o. & as $\mathrm{k}_{5}$ & n.o. \\
\hline $\begin{array}{l}\text { GDP release, } \\
\mathrm{k}_{6}\left(\mathrm{~s}^{-1}\right)\end{array}$ & Bpy-GTP & $5.5 \pm 0.5$ & $7.4 \pm 0.6$ & $7.3 \pm 0.6$ & n.o. \\
\hline $\begin{array}{l}\text { IF2 binding to } 30 \mathrm{~S}\left(2^{\text {nd }}\right. \\
\text { round }), \mathrm{k}_{7}\left(\mathrm{~s}^{-1}\right)\end{array}$ & IF2 $757($ Alx555) & $20 \pm 10$ & $21 \pm 10$ & $16 \pm 9$ & n.o. \\
\hline $\begin{array}{l}\text { Peptide bond } \\
\text { formation, } \mathrm{k}_{8}\left(\mathrm{~s}^{-1}\right)\end{array}$ & fMet-Phe & $2.6 \pm 0.9$ & $2.0 \pm 0.9$ & $2.5 \pm 0.7$ & n.o. \\
\hline
\end{tabular}

All rates and standard errors are derived from global fitting of collective time courses using numerical integration; n.o. - not observed. 
(A)
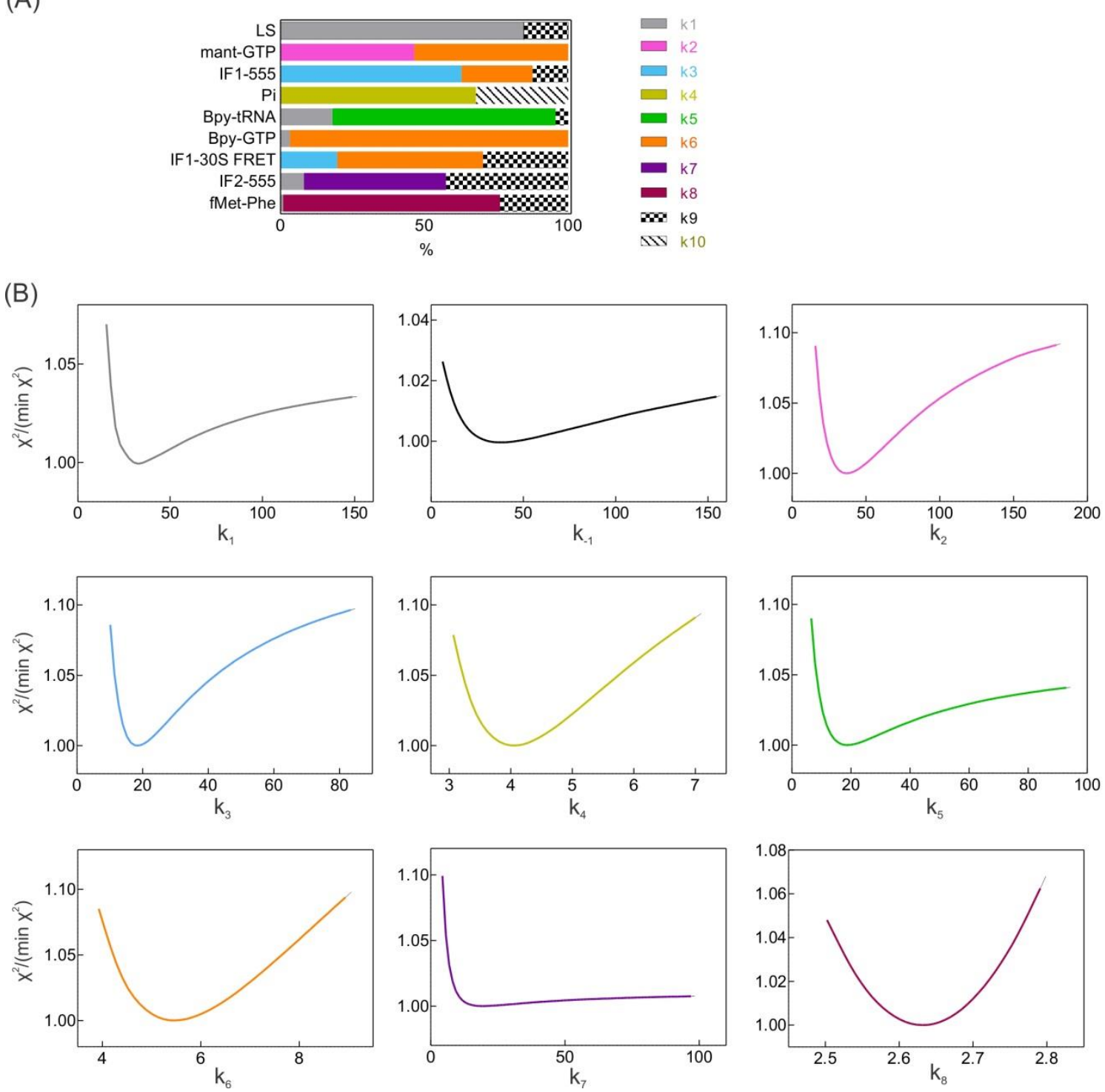

Figure 30. Statistical analysis of global fitting of datasets using KinTek Explorer. Time courses of all observables (LS; FRET between mant-GTP and Trp residue of IF2; fluorescence changes of $\mathrm{IF}_{4}(\mathrm{Al} \times 555)$; $\mathrm{Pi}$ release; fluorescence changes of Bpy-MettRNA ${ }^{\text {fMet; }}$; fluorescence changes of Bpy-GTP; fluorescence changes of IF2 $_{757}(\mathrm{Al} \times 555)$; dequenching of $30 \mathrm{~S}_{\mathrm{S} 13}(\mathrm{Alx} 488)$ by $\mathrm{IF}_{4}(\mathrm{Atto540Q})$ and the first peptide bond formation) were obtained at increasing concentrations of $50 \mathrm{~S}$ subunits, and collectively evaluated by numerical integration using a 10-step kinetic model (see Results). (A) Distribution of amplitudes of the observables along different steps of the model. Amplitudes are presented as \% of signal change at a given step compared to the total change. For all observables where the fluorescence changes go into one direction, the total amplitude is set at $0-100 \%$. For mant-GTP, where the fluorescence change has an upward and downward phase, the amplitudes of both steps were summed up, disregarding the sign. (B) Global minima and confidence intervals of elemental rate constants $\left(\mathrm{s}^{-1}\right)$ derived from global fitting of datasets. 
The association and dissociation elemental rate constants of subunit joining, derived from numerical integration, were $30 \pm 6 \mu \mathrm{M}^{-1} \mathrm{~s}^{-1}$ and $31 \pm 15 \mathrm{~s}^{-1}$, respectively (Table 2). Subunit joining was followed by rapid GTPase activation (elemental rate $-36 \mathrm{~s}^{-1}$ ) and change in IF1 environment $\left(19 \mathrm{~s}^{-1}\right)$. Subsequently, Pi was released from IF2 $\left(4 \mathrm{~s}^{-1}\right)$, followed by tRNA ${ }^{\text {iMet }}$ dissociation from IF2 $\left(19 \mathrm{~s}^{-1}\right)$. Thereafter, IF1 and IF2 dissociation, as well as dissociation of Bpy-GDP from the IF2 G-domain, proceeded around the same time with an elemental rate constant of $5.5 \mathrm{~s}^{-1}$. Finally, the mature $70 \mathrm{~S}$ complex catalyzed the formation of the first peptide bond $\left(2.5 \mathrm{~s}^{-1}\right)$. In parallel, the released IF2 entered the second round of initiation by rapidly binding to free $30 \mathrm{~S}$ subunits $\left(\sim 20 \mathrm{~s}^{-1}\right)$.

The elemental association rate constant of 50 S joining to 30 S complexes lacking IF1 or IF3 was slightly higher $\left(40-50 \mu^{-1} \mathrm{~s}^{-1}\right)$, while the elemental dissociation rate constant was very small $\left(\sim 0-2.5 \mathrm{~s}^{-1}\right)$ and the reaction was rendered almost irreversible (Table 2). When IF3 was absent, the kinetics of GTPase activation was indistinguishable from subunit joining, whereas in the absence IF1, the elemental rate constant of GTPase activation was slightly lower $\left(24 \mathrm{~s}^{-1}\right)$. There was no difference observed in the elemental rate constants of IF1 environment change $\left(17 \mathrm{~s}^{-1}\right)$ and Pi release $\left(4 \mathrm{~s}^{-1}\right)$ when IF3 (or IF1) were lacking from the complex. The absence of either factor slightly decreased the rate of tRNA ${ }^{\text {fMet }}$ release from IF2 $\left(10-13 \mathrm{~s}^{-1}\right)$, which under these conditions took place concomitantly with IF2 (and IF1) dissociation from the 70S complex. Subsequently, GDP release from IF2 became slightly faster $\left(7.3 \mathrm{~s}^{-1}\right)$, while events such as binding of IF2 to $30 \mathrm{~S}$ during the second round of initiation $\left(\sim 20 \mathrm{~s}^{-1}\right)$ and the first peptide bond formation $\left(\sim 2 \mathrm{~s}^{-1}\right)$ were not affected. 


\subsection{Reversibility of 70 S IC formation}

To further examine the role of GTP hydrolysis during 70S IC formation, we investigated whether a mature 70 S IC can recruit IF1 and IF2 when GTP is replaced with GTPYS.

\subsubsection{Binding of IF2 to mature 70 S IC}

As noted above, when 30S IC formed with GTP and Bpy-Met-tRNA ${ }^{\text {fMet }}$ was rapidly mixed with 50S subunits, a decrease in Bpy fluorescence was observed due to the release of Bpy-MettRNA ${ }^{\text {fMet }}$ from IF2 following GTP hydrolysis (Fig. 16 and Fig. 31A). When the same experiment was performed in the presence of a 20 -fold excess of GTPYS added along with the $50 \mathrm{~S}$ subunits, a biphasic fluorescence change was observed (Fig. 31A). The initial decrease in signal was followed by an increase in fluorescence caused by re-binding of IF2, after the exchange of GDP for GTPYS, to the mature 70 S IC and subsequent capture of the 3' CCA-end of Bpy-Met-tRNA ${ }^{\text {fMet }}$ by IF2-GTPYS. Thus, in the absence of GTP hydrolysis, the binding equilibrium is shifted towards the formation of the Bpy-Met-tRNA ${ }^{\mathrm{fMet}}-\mathrm{IF} 2-70 \mathrm{~S}$ complex and the dissociation of IF2 is disfavored, thereby preventing the formation of the mature 70 S IC which can enter the elongation phase. 

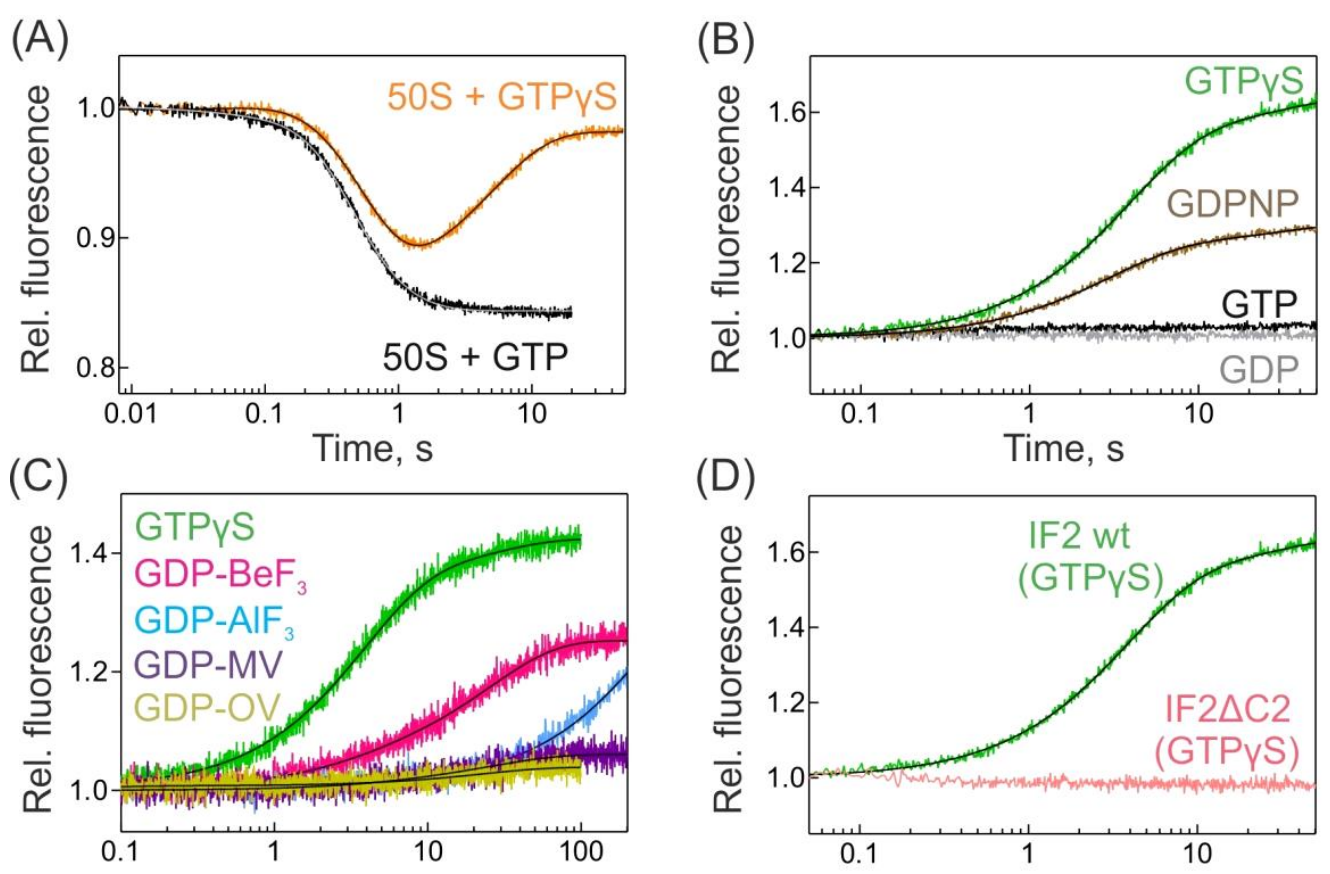

Figure 31. Binding of IF2 to mature 70S IC in the presence of pre- and post-hydrolysis state GTP analogs. (A) $30 \mathrm{~S} \mathrm{IC} \mathrm{formed} \mathrm{in} \mathrm{the} \mathrm{presence} \mathrm{of} \mathrm{Bpy-Met-tRNA}{ }^{\text {fMet }}$ and GTP (12.5 $\mu \mathrm{M})$ was rapidly mixed with $50 \mathrm{~S}$ subunits in the presence or absence of GTPYS $(0.25 \mathrm{mM})$. Time courses of Bpy-Met-tRNA ${ }^{\text {tMet }}$ fluorescence changes were monitored. (B-C) Time courses of Bpy-Met-tRNA ${ }^{\text {fMet }}$ interaction with IF2 C2-domain upon binding of the factor $(4 \mu \mathrm{M})$ to purified 70S IC (Bpy) in the presence of different GTP analogs $(\geq 100 \mu \mathrm{M})$. (D) Similar experiments were performed using an IF2 variant lacking the C2-domain (IF2 $\Delta$ C2) in the presence of GTPYS. Time courses were fit with a double-exponential function and the apparent rate constant of the predominant phase is reported (see text); error bars represent standard error of the fit.

The addition of GDPNP-bound IF2 to preformed 70S IC results in the formation of a high affinity complex between the factor and the ribosome (Antoun et al., 2003). We used this approach to study the reversibility of late events of translation initiation by rapidly mixing IF2, bound to different pre- and post-hydrolysis state GTP analogs, with purified 70S IC containing Bpy-Met-tRNA ${ }^{\mathrm{fMet}}$ (70S IC (Bpy)). In the presence of GTPYS or GDPNP, an increase in fluorescence $\left(0.3 \mathrm{~s}^{-1}\right)$, depicting the binding of IF2 to Bpy-Met-tRNA ${ }^{\text {fMet }}$ on the ribosome, was observed (Fig. 31B). No fluorescence increase occurred upon binding of the full-length IF2 to 70S IC (Bpy) in the presence of GTP, owing to rapid GTP hydrolysis and IF2 dissociation. Control experiments performed in the presence of GDP also showed no fluorescence change.

In the presence of GDP- $\mathrm{BeF}_{3}$ and $\mathrm{GDP}-\mathrm{AlF}_{3}$ (Fig. 31C), a 7-70 fold slower fluorescence change, than that observed in the case of GTPYS and GDPNP, was noted $\left(0.04 \mathrm{~s}^{-1}\right.$ and 
$0.004 \mathrm{~s}^{-1}$, respectively). No significant increase in fluorescence was observed when GDPortho-vanadate or GDP_-meta-vanadate were used instead. When a truncated version of IF2, lacking the C2-domain (Mortensen et al., 1998), was used together with GTPYS, no fluorescence change was observed, confirming the loss of the direct interaction between tRNA ${ }^{\text {fMet }}$ and IF2 (Fig. 31D).

\subsubsection{Binding of IF1 to mature $70 \mathrm{~S} I \mathrm{C}$}

When $30 \mathrm{~S}_{\mathrm{S} 13}$ (Alx488) IC, formed with GTP and IF1 ${ }_{4}$ (Atto540Q), was rapidly mixed with $50 \mathrm{~S}$ subunits, an increase in fluorescence was observed upon release of $\mathrm{IF}_{4}$ (Atto540Q) from the $70 \mathrm{~S}$ complex (Fig. 24 and Fig. 32A). In the presence of a 20 -fold excess of GTPYS added along with the $50 \mathrm{~S}$ subunits, a biphasic fluorescence change was observed (Fig. 32A) representing the initial release of IF1, followed by rebinding of the factor to the $70 S$ complex. Whether externally-added IF1 could bind to mature 70 S IC was checked by mixing unpurified $70 \mathrm{~S}_{\mathrm{S} 13}$ (Alx488) IC (which contained IF2 in solution) with $\mathrm{IF}_{4}{ }_{4}$ (Atto540Q) in the presence of GTP or GTPYS (Fig. 32B). No binding was observed in the presence of GTP, whereas IF1 could bind to mature $70 \mathrm{~S}$ complexes in the presence of GTPYS
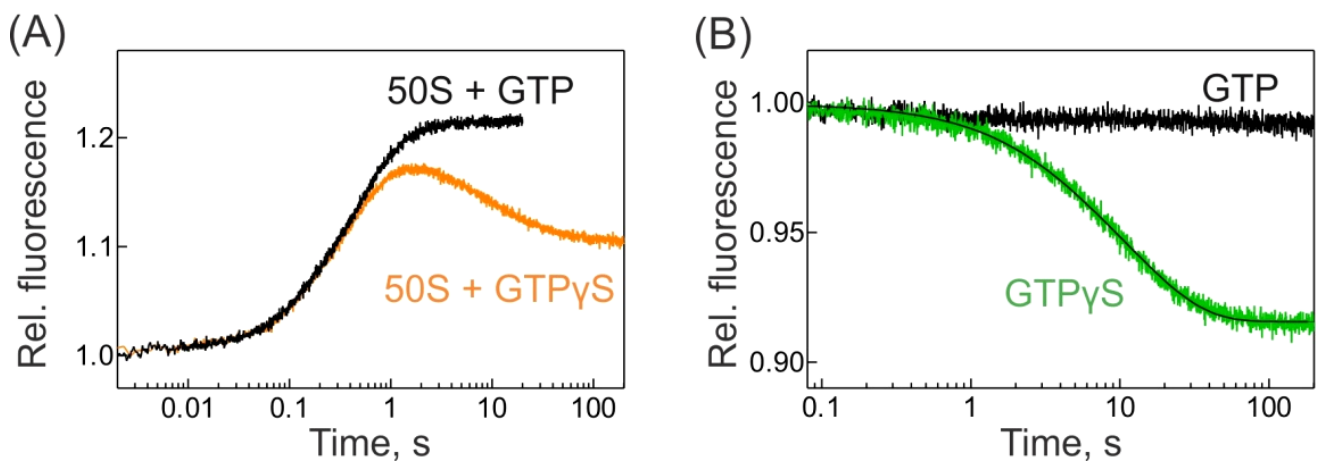

Figure 32. Binding of IF1 to mature $70 \mathrm{~S} \mathrm{IC}$ in the presence of GTPYS. (A) $30 \mathrm{~S}_{\mathrm{S} 13}(\mathrm{Al} \times 488$ ) IC, formed with $\mathrm{IF}_{4}($ Atto540Q) and GTP $(12.5 \mu \mathrm{M})$ was rapidly mixed with $50 \mathrm{~S}$ subunits in the presence or absence of GTPYS $(0.25 \mathrm{mM})$. Time courses of FRET were monitored. (B) Time courses of $\mathrm{IF}_{4}\left(\right.$ Atto540Q) $(0.1 \mu \mathrm{M})$ binding to mature $70 \mathrm{~S}_{\mathrm{S} 13}$ (Alx488) IC in the presence of GTP or GTPYS $(0.25 \mathrm{mM})$.

Because IF1 does not harbor a nucleotide binding site, the recruitment of IF1 to the 70S complex must be indirectly mediated by the effector molecule, IF2, which binds to the mature 70 S IC in the presence of GTPYS and shifts the equilibrium towards the pre-hydrolysis state of the 705 complex. 


\section{DISCUSSION}

\subsection{Kinetic model of 70S IC formation}

The present kinetic analysis provides a detailed mechanistic picture of the maturation of the canonical 30S IC to the 70S EC (Fig. 33).

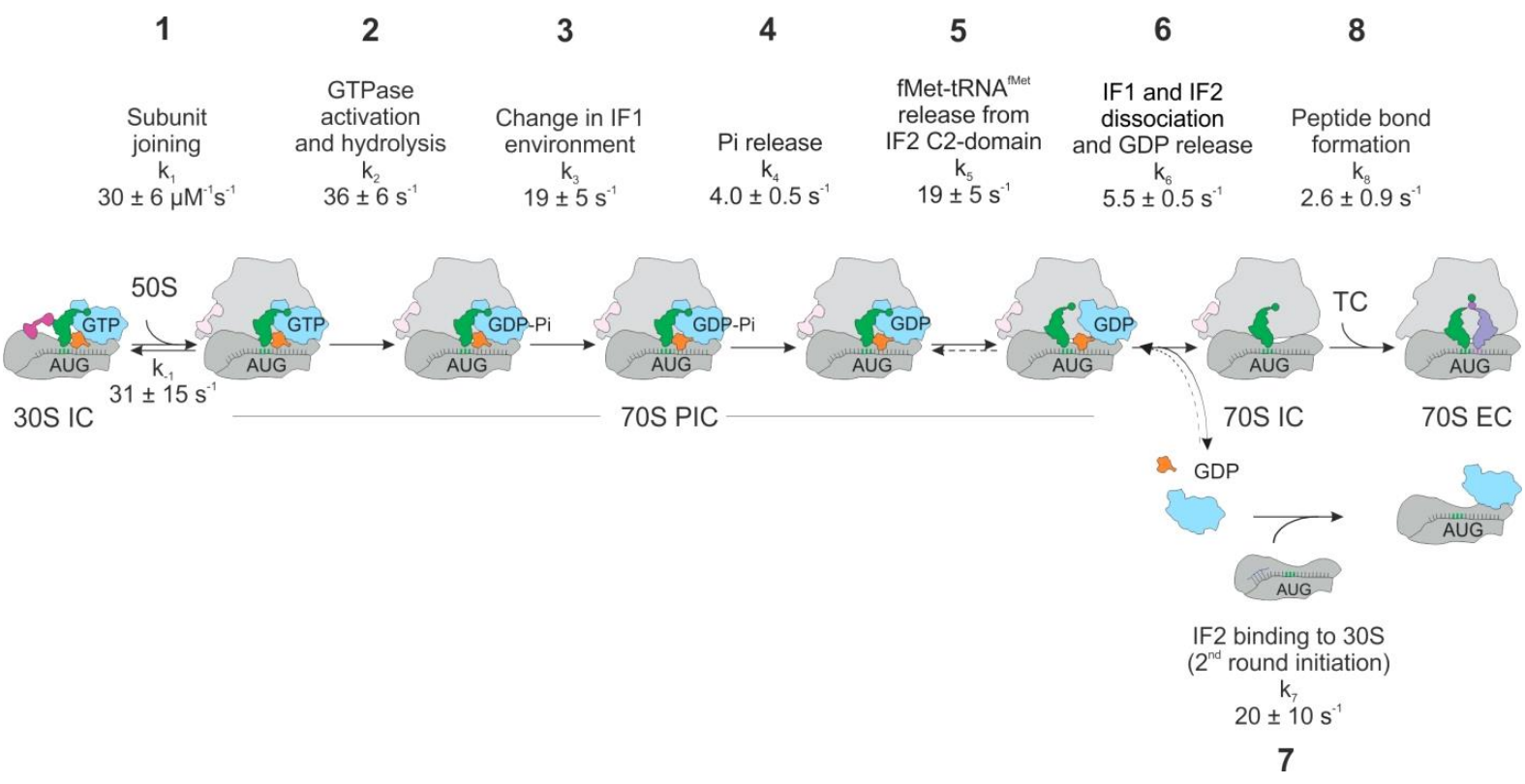

Figure 33. Detailed kinetic scheme of late events in bacterial translation initiation. IF1, IF2-GTP, IF3, mRNA and fMet-tRNA ${ }^{\text {fMet }}$ bind the 30 S subunit to form a 30 S IC. Step 1: Association of the 50S subunit with $30 \mathrm{~S} \mathrm{IC} \mathrm{to} \mathrm{form} \mathrm{a} \mathrm{70S} \mathrm{PIC.} \mathrm{Step} \mathrm{2:} \mathrm{GTPase} \mathrm{activation} \mathrm{and}$ rapid GTP hydrolysis (Grigoriadou et al., 2007a; Huang et al., 2010; Qin et al., 2009; Tomsic et al., 2000). Step 3: Change of IF1 environment. Step 4: Pi release from IF2. Step 5: Release of the 3' end of fMet-tRNA $A^{\text {fMet }}$ from IF2 C2-domain. Step 6: Release of IF2 from the 70 complex and GDP from IF2; release of IF1 from the 70S complex, giving rise to an elongation ready 70S IC. Step 7: Binding of IF2 to free 30S subunits in the next round of initiation. Step 8: Binding of EF-Tu-GTP-aminoacyl-tRNA (TC) to the 70S IC is followed by peptide bond formation to form a 70S EC. Movement of IF3 away from the subunit interface takes place immediately upon subunit joining; the position of IF3 is indicated by a lighter shade and should be considered tentative. Dissociation of IF1 and IF2 from the 70S complex, as well as step 5, becomes reversible in the absence of GTP hydrolysis, as indicated by dashed arrows.

$50 \mathrm{~S}$ subunit joining is the first step towards the formation of the $70 \mathrm{~S}$ complex (Antoun et al., 2006b; Antoun et al., 2004; Grunberg-Manago et al., 1975; Milon et al., 2008). When all factors, fMet-tRNA ${ }^{\mathrm{fMet}}$, and GTP are bound to the 30S subunit carrying the 022 mRNA,

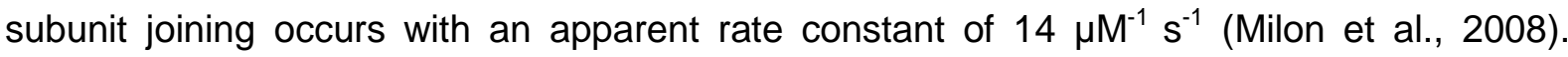
However, the detailed kinetic analysis, which takes into account the steps following $50 \mathrm{~S}$ subunit joining, indicates that the initial $50 \mathrm{~S}$ subunit docking is reversible with elemental rate 
constants of about $k_{1}=30 \mu \mathrm{M}^{-1} \mathrm{~s}^{-1}$ and $\mathrm{k}_{-1}=31 \mathrm{~s}^{-1}$ (Table 2), consistent with previously published results (Grigoriadou et al., 2007a). The 70S PIC intermediate formed immediately upon $50 \mathrm{~S}$ subunit joining (Fig. 33) may correspond to the short-lived state of the $70 \mathrm{~S}$ complex observed by single-molecule FRET (MacDougall and Gonzalez, 2015). Further rearrangements of the complex are required to stabilize the interaction between the two subunits, leading to the formation of the 70S IC (Grigoriadou et al., 2007a; MacDougall and Gonzalez, 2015).

Because IF3 binds to the principle inter-subunit bridge B2b which is essential for stable subunit association (Dallas and Noller, 2001), docking of the 50S subunit displaces IF3 from the interface between the subunits (Fig. 28C), leading to the eventual dissociation of the factor. These results are in agreement with previous studies conducted using rapid kinetics (Milon et al., 2008), chemical probing (Fabbretti et al., 2007), and single molecule FRET (Elvekrog and Gonzalez, 2013; MacDougall and Gonzalez, 2015) which suggest that IF3 dissociation from the ribosome occurs during or after $50 \mathrm{~S}$ subunit joining.

Subunit joining is followed by rapid GTPase activation of IF2 (36 s $)$ and GTP hydrolysis (Grigoriadou et al., 2007a; Huang et al., 2010; Qin et al., 2009; Tomsic et al., 2000), along with subsequent conformational rearrangements resulting in a change of IF1 environment $\left(19 \mathrm{~s}^{-1}\right)$ (Fig. 33; Table 2). Thereafter, $\mathrm{Pi}$ is released from the G-domain of IF2 $\left(4 \mathrm{~s}^{-1}\right)$ after a delay of $\sim 75-100 \mathrm{~ms}$, in agreement with earlier reports (Grigoriadou et al., 2007a; Tomsic et al., 2000). Pi release is followed by the rapid release of fMet-tRNA ${ }^{\text {fMet }}$ from the C2-domain of IF2 $\left(19 \mathrm{~s}^{-1}\right.$ ), leading to tRNA accommodation in the P site (Allen et al., 2005; La Teana et al., 1996; Myasnikov et al., 2005). Previous reports showed that tRNA ${ }^{\text {fMet }}$ conformational changes, monitored by the fluorescence change of proflavin attached to the D-loop of fMet$\mathrm{tRNA}^{\mathrm{fMet}}$, precede Pi release (Grigoriadou et al., 2007a). The datasets with Bpy-Met-tRNA ${ }^{\mathrm{fMet}}$ could not be modeled to suit this sequence of events, suggesting that the two labels may report on two different tRNA ${ }^{\text {fMet }}$-dependent reactions.

Afterwards, IF2 dissociates from the 70 S complex $\left(5.5 \mathrm{~s}^{-1}\right)$ and exchanges its bound GDP for GTP to participate in further initiation events. Because the final concentration of GDP in the reaction, after GTP hydrolysis, is determined by the concentration of the 30S IC $(0.1 \mu \mathrm{M})$, which is at least 10-fold lower than the $\mathrm{K}_{d}$ of GDP binding to free-IF2 ( 1-2 $\mu \mathrm{M}$ (Hauryliuk et al., 2009)), the dissociation of GDP from IF2 is expected to be spontaneous. It has been reported that, after GTP hydrolysis and Pi release, IF2 may remain bound to the $70 \mathrm{~S}$ complex posing no hindrance to the binding of the incoming EF-Tu ternary complex 
(Grigoriadou et al., 2007a). However, the entry of IF2 into the $2^{\text {nd }}$ round of translation initiation $\left(20 \mathrm{~s}^{-1}\right)$ (Fig. 20) indicates that IF2 completely dissociates from the $70 \mathrm{~S}$ complex after release from $\mathrm{FMet}^{-\mathrm{TRNA}} \mathrm{A}^{\mathrm{fMet}}$. IF2 binding to the $30 \mathrm{~S}$ subunit does not require interaction with GTP (Weiel and Hershey, 1982) or fMet-tRNA ${ }^{\text {fMet }}$. However, the binding of GTP and fMet-tRNA $^{\text {fMet }}$ stimulates conformational changes in IF2 (Fig. 19B-C), conferring an 'active' conformation on the factor and promoting a productive arrangement of the $30 \mathrm{~S}$ IC which is primed for rapid subunit joining.

Until now, relatively little was known about the timing of IF1 release during 70S IC formation. Cryo-EM structures suggested that it remains bound to the $70 \mathrm{~S}$ complex after $50 \mathrm{~S}$ joining, positioned near IF2 helix 8, which connects the G3- and C1 domain of IF2 (Allen et al., 2005). Release of IF1 was suggested to occur sometime after $50 \mathrm{~S}$ joining but before $\mathrm{Pi}$ release, allowing the IF2 CTD to reposition itself near the decoding center of the ribosome (Allen et al., 2005; Myasnikov et al., 2005). Numerical integration analysis of time courses monitoring IF1 dynamics revealed that IF1 dissociates from the 70 S complex after Pi release and around the same time as IF2 (Table 2).

Lastly, EF-Tu TC binds to the 70S IC and peptide bond formation occurs after a $300 \mathrm{~ms}$ lag phase with the elemental rate constant of $2.6 \mathrm{~s}^{-1}$, marking the transition of initiation into elongation phase of translation. The delay represents the time required for the formation of an elongation-competent $70 \mathrm{~S}$ complex. When pre-formed $70 \mathrm{~S} \mathrm{IC} \mathrm{is} \mathrm{used} \mathrm{instead,} \mathrm{no} \mathrm{delay}$ phase is observed, and the time courses of peptide bond formation can be fit with a singleexponential function which gives a rate of $2 \mathrm{~s}^{-1}$ (Milon et al., 2008; Pape et al., 1998), in agreement with the rates derived from the numerical integration model. 


\subsection{Role of GTP hydrolysis in maturation of the 70 S complex}

The requirement for GTP hydrolysis by IF2, in promoting different reactions, was examined by substituting GTP with a non-hydrolysable GTP analog, GTPYS. In agreement with previous reports, the replacement of GTP with non-hydrolysable GTP analogs did not affect the rate or extent of subunit joining (Fig. 12B) (Antoun et al., 2003; Antoun et al., 2004; Qin et al., 2009; Tsai et al., 2012). Because 50S docking displaces IF3 from the subunit interface, the latter reaction was also entirely independent of GTP hydrolysis (Fig. 28D). These findings contradict the conclusions from cryo-EM studies of the 70S PIC, where the complex is stalled in the presence of non-hydrolysable GTP analog, GDPNP (Allen et al., 2005). The authors assign a density found near the E site to IF3, suggesting that GTP hydrolysis may be required for IF3 release from the $70 \mathrm{~S}$ complex (Allen et al., 2005). Taking into account these observations, it is possible that the step assigned to IF3 dissociation in this work represents a major movement of IF3, e.g. away from the subunit interface and towards the outer surface of the ribosome (Fig. 33), which would free the bridge B2b (Dallas and Noller, 2001; Hennelly et al., 2005; Julian et al., 2011; Kipper et al., 2009) together with other inter-subunit bridges (Fabbretti et al., 2007), and allow stable subunit interaction to take place.

GTPase activation of IF2 could take place even in the presence of a non-hydrolysable GTP analog, mant-GTPYS (Fig. 13D). In contrast, the replacement of GTP with GTPYS completely abolished the release of tRNA ${ }^{\text {fMet }}$ from the C2-domain of IF2 (Fig. 16D). The latter finding is in agreement with the cryo-EM reconstruction of the 70S PIC where the physical interaction between fMet-tRNA ${ }^{\text {fMet }}$ and IF2 remains unbroken when complexes are formed in the presence of GDPNP (Allen et al., 2005). In agreement with previous reports based on biochemical, structural and rapid-kinetic data, IF2 was unable to dissociate from the $70 \mathrm{~S}$ ribosome, in the absence of GTP hydrolysis (Fig. 13D and Fig. 20D) (Allen et al., 2005; Antoun et al., 2003; Lockwood et al., 1972; Luchin et al., 1999; Myasnikov et al., 2005). Peptide bond formation was heavily dependent on GTP hydrolysis as well (Fig. 29D) (Antoun et al., 2003).

The utilization of GTPYS and GDPNP (Antoun et al., 2003) conferred a higher degree of inhibition than was previously reported in the presence of GDPCP (Grigoriadou et al., 2007a; Grigoriadou et al., 2007b), or GDP/no nucleotide (Tomsic et al., 2000). In the former case, the authors (Grigoriadou et al., 2007a; Grigoriadou et al., 2007b) observed only a 2-3 fold reduction in the amplitude of tRNA ${ }^{\text {imet }}$ conformational changes and dipeptide formation, events that were almost completely inhibited in this work. In addition, cryo-EM structures of 
the 70 s complex, prepared in the presence of GDPCP, depicted a loss of interaction between IF2 and tRNA ${ }^{\text {fMet }}$, even in the absence of GTP hydrolysis (Myasnikov et al., 2005). To understand the discrepancy, the subunit joining and tRNA ${ }^{\text {fMet }}$ release from IF2 was checked in the presence of the same analog, GDPCP, and very little differences were found when compared to GTPYS (Fig. 12B and 16D). It was also observed that the rate of subunit joining in the absence of any nucleotide or in the presence of GDP $\left(0.02 \mathrm{~s}^{-1}\right)$ was $>200$-fold slower than in the presence of GTP (Fig. 6E and Fig. 12A), in agreement with earlier reports (Antoun et al., 2004). These results suggest that the disparity may arise from variations in the susceptibility of IF2 from different organisms (E. coli (Antoun et al., 2003; Antoun et al., 2004); this work), G. stearothermophilus (Grigoriadou et al., 2007a; Grigoriadou et al., 2007b; Tomsic et al., 2000) and T. thermophilus (Myasnikov et al., 2005)) for different nonhydrolysable analogs of GTP.

It remains unknown whether Pi release is an obligatory step for the release of fMet-tRNA ${ }^{\text {fMet }}$ from IF2. To investigate this, the effect of phosphate analogs on IF2 interaction with the tRNA ${ }^{\text {fMet }}$ was studied. Because the phosphate analogs affected the conformational state of IF2 on the $30 \mathrm{~S} \mathrm{IC}$, disturbances in subunit joining were observed (Fig. 12C-D). Hence, the reverse pathway was monitored, in the sense that when IF2, bound to different pre- and post-hydrolysis GTP analogs, was mixed with mature 70S IC (Bpy), it could bind to the 70S IC and interact with the Bpy-labeled 3' CCA-end of tRNA ${ }^{\text {tMet }}$. In the presence of prehydrolysis state GTP analogs such as GTPYS, GDP- $\mathrm{BeF}_{3}$ and GDP-AIF $F_{3}$ IF2 could bind tRNA $^{\text {fMet }}$ (Fig. 31C). However, when post-hydrolysis state analogs such as GDP—orthovanadate and GDP_-meta-vanadate were used, which should represent the GDP-Pi conformation of IF2, no interaction was observed between IF2 and tRNA ${ }^{\mathrm{fMet}}$. At first glance, these results would indicate that tRNA ${ }^{\mathrm{fMet}}$ dissociation from IF2 is independent of Pi release because, in its supposed GDP-Pi conformation, IF2 could not revert the 70 S complex to a state where tRNA ${ }^{\text {fMet }}$ interacts with IF2. However, it should be kept in mind that working with vanadates can pose several technical challenges which may lead to ambiguous data interpretation. Vanadates have a tendency to form polymeric species in solution near neutral $\mathrm{pH}$. Since the reactions were performed at the near-physiological $\mathrm{pH}$ of 7.4, it cannot be excluded that unreactive polymeric vanadate species may have resulted in the lack of a productive reaction. A second technical challenge is that vanadates may form a cyclic covalent complex with the nucleotide which does not represent the expected post-hydrolysis 
GTP state (reviewed in (Davies and Hol, 2004)). Thus, on the basis of these results, the importance of $\mathrm{Pi}$ release for $\mathrm{TRNA}^{\mathrm{fMet}}$ dissociation from IF2 remains uncertain.

GTP hydrolysis by IF2 and EF-G produces similar conformational changes in the respective factor and the ribosome. In case of EF-G, Pi release and tRNA-mRNA translocation can take place independently of one another (Savelsbergh et al., 2003); a similar mechanism may apply to $\mathrm{Pi}$ and $\mathrm{fMet}^{-\mathrm{RRNA}} \mathrm{fMet}^{\mathrm{fm}}$ release from IF2. If the two factors employ a similar coupling mechanism, then a step preceding $\mathrm{Pi}$ release and tRNA ${ }^{\text {fMet }}$ dissociation from IF2 may be rate-limiting for the remaining part of the IF2 pathway. On the other hand, if $\mathrm{Pi}$ release is necessary for fMet-tRNA ${ }^{\text {fMet }}$ dissociation from IF2, it would imply that the G-domain can convey conformational changes to the C2-domain. It is not clear how GTP hydrolysis or Pi release from the G-domain can be communicated to the C2-domain of IF2. While structural work on the eukaryotic/archaeal IF2 homolog, e/alF5B, suggested that the nucleotide binding status of the molecule may be communicated through the inter-domain interface (Kuhle and Ficner, 2014a; Roll-Mecak et al., 2000), IF2 might not use the same mechanism because of the different arrangement of its domains (Eiler et al., 2013; Wienk et al., 2012). Pi and tRNA $^{\mathrm{fMet}}$ release from IF2 promote dissociation of the factor from the ribosome via (i) the conformational rearrangement of IF2 from its high-affinity state on the $70 \mathrm{~S}$ complex to its low-affinity, ready-to-leave GDP conformation (Allen et al., 2005; Myasnikov et al., 2005),

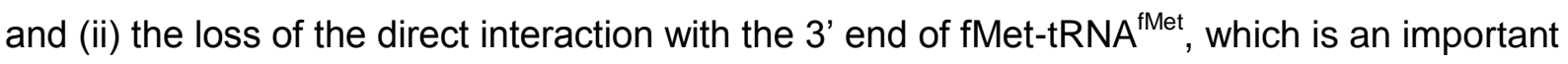
anchor point for IF2 on the ribosome (Allen et al., 2005; Simonetti et al., 2008). In fact, it has been observed that a lower affinity of IF2 towards fMet-tRNA ${ }^{\text {fMet }}$ ((Gualerzi et al., 2001); Akanksha Goyal - master thesis) or the ribosome (Fabbretti et al., 2012; Shin et al., 2002) can help bypass the requirement for GTP hydrolysis in promoting IF2 release from the $70 \mathrm{~S}$ complex.

The two observables used to monitor IF1 dynamics on the $70 \mathrm{~S}$ complex, were influenced to different extents by the lack of GTP hydrolysis. The absence of GTP hydrolysis did not affect the change in IF1 environment after subunit association (Fig. 22D), but surprisingly, IF1 dissociation from the 70S complex was largely prevented (Fig. 24C). The dissociation of IF1 from the $70 S$ complex may be promoted by the loss of direct interaction with IF2 or rearrangements of the ribosome that occur after GTP hydrolysis. It is unclear which of the several IF2-dependent events, such as GTP hydrolysis, Pi release, fMet-tRNA ${ }^{\text {fMet }}$ release, inter-subunit rotation, or the dissociation of IF2 from the ribosome, is directly responsible for promoting the release of IF1 from the 70 S IC. Because the dependence of IF1 release from 
the 70 S complex on GTP hydrolysis was not eliminated by using an IF2 variant lacking the NTD (Fig. 25B), it is likely that the release of IF1 is dependent on GTP hydrolysis even in those organisms in which IF2 does not retain its full-length NTD.

Once released, IF1 could re-bind only to the pre-hydrolysis state of the ribosome (induced by IF2-GTPYS) (Fig. 32). It is likely that IF2 binding to the 70S complex in the presence of nonhydrolysable GTP analogs confers a pre-hydrolysis conformation on the $70 \mathrm{~S}$ complex, revealing an IF1 binding site. Hence, the dissociation of IF1 from the $70 \mathrm{~S}$ complex may be promoted by a conformational rearrangement of the ribosome which occurs upon GTP hydrolysis and IF2 dissociation. The presence of a non-hydrolysable GTP analog shifted the equilibrium towards a stable $70 S$ - mRNA-IF1-IF2—fMet-tRNA ${ }^{\mathrm{fMet}}$ complex, suggesting that GTP hydrolysis may guide the unidirectional progression of the 705 complex into an elongation-competent state, by rendering the dissociation of IF2 and IF1 from the ribosome irreversible. 


\subsection{Interplay between Initiation factors}

On the 30S IC, IF1 and IF3 strongly stabilize each other's binding by modulating the conformation of the $30 \mathrm{~S}$ subunit (Antoun et al., 2006b; Milon et al., 2012; Zucker and Hershey, 1986). As a result, IF1 and IF3 dissociation from the $30 \mathrm{~S}$ complex became 4-8 fold faster in each other's absence (Fig. 23B and Fig. 27B). The two factors influence the rate of $50 S$ subunit joining by affecting the relative arrangement of the 30 S subunit, IF2 and fMettRNA $^{\mathrm{fMet}}$, and conferring an anti-association conformation to the $30 \mathrm{~S}$, which is strengthened or relieved depending on the mRNA properties and codon-anticodon interaction between the mRNA and fMet-tRNA ${ }^{\text {fMet }}$ (Antoun et al., 2006b; Milon et al., 2008).

As mentioned previously, when an mRNA containing an optimal TIR (022 mRNA used in this work) is used to form complexes, subunit association occurs rapidly with an apparent rate of $14 \mu \mathrm{M}^{-1} \mathrm{~s}^{-1}$. Significantly slower apparent rates of subunit joining $\left(0.2 \mathrm{~s}^{-1}\right)$ are observed when complexes are formed using mRNAs containing a non-canonical TIR (Grigoriadou et al., 2007b; Milon et al., 2008) or the 002 mRNA which contains a non-optimal TIR consisting of a long (9-nt) SD sequence followed by a short (5-nt) spacer between the SD sequence and the AUG start codon (Calogero et al., 1988; Milon et al., 2008). It is likely that the low apparent rate of subunit joining is due to the increased dissociation of 'unproductive' complexes into individual subunits and slow transitions towards the 70S EC (Grigoriadou et al., 2007a; Milon et al., 2008). In the absence of either IF1 or IF3, the elemental rate of subunit association was slightly higher $\left(40-50 \mu \mathrm{M}^{-1} \mathrm{~s}^{-1}\right)$, but the dissociation of the complex was very slow (Table 2), giving rise to the overall higher apparent rate constants of the reaction $\left(\sim 40 \mu \mathrm{M}^{-1} \mathrm{~s}^{-1}\right)$. The low rates of subunit dissociation indicate the formation of a longer-lived complex, explaining how IF1 and IF3 contribute to mRNA selection at the 50 S subunit association step (MacDougall and Gonzalez, 2015; Milon et al., 2008).

The faster subunit joining, in the absence of either factor, resulted in higher apparent rates of subsequent reactions without affecting their elemental rate constants. Under these conditions, the dissociation of IF2 and IF1 occurred around the same time as the release of tRNA $^{\mathrm{fMet}}$ from IF2 C2-domain (Table 2). Other than that, the lack of IF1 did not significantly affect the timing or extent of the remaining IF2-dependent reactions on the 70S complex. The absence of IF3 from the system also did not affect the timing of these IF2-dependent processes. It did, however, lower the tRNA fluorescence and peptide bond formation to by 20-40\%, suggesting that in the absence of IF3, a fraction of the 70S complexes did not carry fMet-tRNA $^{\mathrm{fMet}}$. Overall, the IF2 pathway followed the same sequence of events, regardless of 
the presence of IF1 or IF3 (Fig. 34). In summary, 50 S subunit joining was the only step in the late initiation pathway influenced by the absence of IF1 or IF3, whereas none of the following steps were significantly affected.

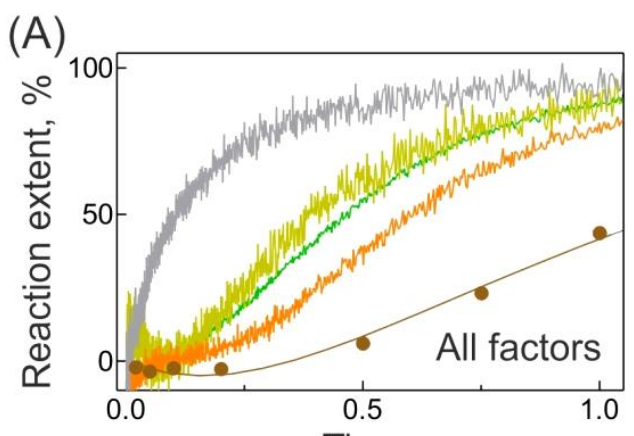

(B)

Time, $s$

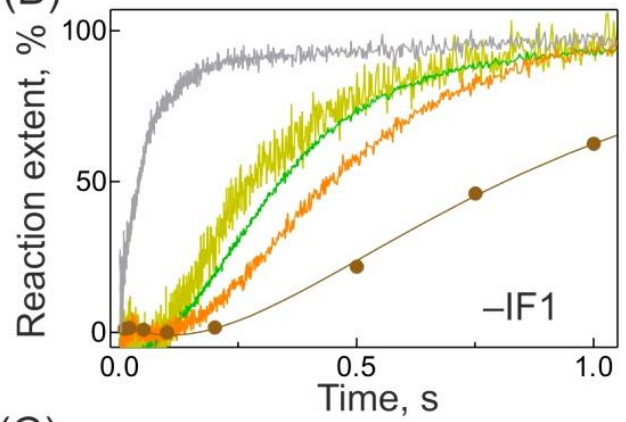

(C)

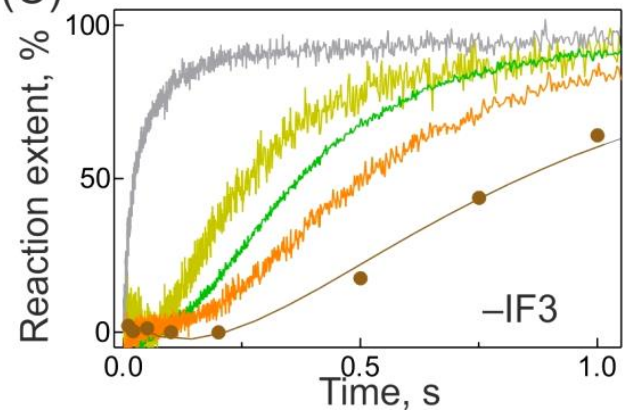

Figure 34. Direct comparison of reaction time courses during 70 S IC formation. Time courses of subunit association (grey), Pi release (gold), tRNA release from IF2 (green), GDP release (orange) and peptide bond formation (brown) are presented at $1 \mu \mathrm{M} 50 \mathrm{~S}$ subunit concentration. All traces were normalized with respect to amplitude changes to facilitate visual comparison of time courses. The reactions were monitored in the presence of $(A)$ all factors, or (B) in the absence of IF1, or (C) in the absence of IF3.

The frequency with which an mRNA enters the translational cycle is dependent on the assembly of a $30 \mathrm{~S} \mathrm{IC}$ with a favorable conformation which promotes facile binding of the $50 \mathrm{~S}$ subunit (Milon et al., 2008; Milon et al., 2012). IF1 and IF3 act as gate-keepers during early stages of initiation by preventing subunit docking to unproductive $30 \mathrm{SICs}$ and sustaining a stage in initiation during which the mRNA and tRNA interaction with the ribosome is 
reversible. After stable subunit joining, this checkpoint is crossed and the mRNA can no longer freely dissociate from the complex. It appears that neither IF1 nor IF3 is essential in promoting 70S IC maturation after the 50S subunit docks on a correctly-formed 30 S IC. However, the prolonged retention of IF1 and IF3 on the 70S complex, perhaps due to an incorrectly formed 70S complex, may hinder IF2-related reactions and prevent formation of key stabilizing inter-subunit bridges (Allen et al., 2005; Myasnikov et al., 2005), thus acting as a safeguard against formation of a mature aberrant 70 S IC. 


\subsection{Comparisons with eukaryotic translation initiation and future perspectives}

In eukaryotes, the process of translation initiation is significantly more complex than in bacteria. The canonical pathway is a multi-step process which employs at least 11 eukaryotic initiation factors (elF). Briefly, the small ribosomal subunit, carrying elFs and initiator tRNA, is recruited to the 5' end of the mRNA. The complex then scans the length of the mRNA in a 5' to 3' direction, searching for the first AUG start codon. After codon recognition, the large subunit joins and all elFs are released giving rise to an elongation competent $80 \mathrm{~S}$ IC which can participate in peptide bond formation (reviewed in (Jackson et al., 2010)).

The eukaryotic homologs of IF1, IF2 and IF3 are elF1A, elF5B and elF1, respectively. In light of the evolutionary conservation, it is likely that certain mechanistic aspects of bacterial translation initiation can be extrapolated to higher eukaryotes. Even though the early stages of initiation are very different between the two life domains, some similarities during the late stages have been noted. After start codon recognition, the affinity of elF1 towards the small ribosomal subunit greatly decreases, leading to the factor's release from the complex (Maag et al., 2005). This situation is comparable to that in bacteria, where the affinity of IF3 to the 30S IC is much lower after start codon selection (Milon et al., 2012), even though the factor only moves away from the subunit interface after being displaced by the $50 \mathrm{~S}$ subunit (Elvekrog and Gonzalez, 2013; Fabbretti et al., 2007; MacDougall and Gonzalez, 2015; Milon et al., 2008). In eukaryotes, the initiator tRNA is recruited to the ribosome by a hetero-trimeric GTPase, namely elF2 which is not homologous to the bacterial IF2. GTP hydrolysis and Pi release from elF2 takes place upon start codon recognition and promotes the release of the factor from the complex (Algire et al., 2005), a prerequisite for rapid subunit association which is mediated by the GTP-bound state of elF5B (Pestova et al., 2000). As in case of IF2, subunit joining triggers GTP hydrolysis by elF5B which thereafter dissociates from the $80 \mathrm{~S}$ complex, presumably due to the lower affinity of its GDP-bound form for the ribosome (Pestova et al., 2000; Shin et al., 2002). Finally, mimicking the dependence of IF1 dissociation on IF2-dependent GTP hydrolysis, the absence of GTP hydrolysis by elF5B lowers the rate of elF1A release from the 80 S IC by 10 -fold (Acker et al., 2009).

Until now, insights into eukaryotic translation initiation have been derived predominantly from genetic screens and biochemical assays performed under steady-state conditions. The large amount of purified components required to carry out fluorescence-based rapid kinetic experiments, has posed a hindrance for monitoring the process in real time. Several elFs which are multi-subunit in composition can be purified only from cell-extracts and are 
obtained in very small quantities (Pisarev et al., 2007). As such, significant progress has been made by Lorsch and colleagues who have not only established protocols for purifying multi-subunit elFs from Saccharomyces cerevicae, expressed recombinantly in bacteria (Acker et al., 2007), but have also successfully utilized rapid-kinetic approaches to study presteady state initiation events in lower eukaryotes. However, such procedures have not yet been established for mammalian elFs and the purchase of large quantities of cell extracts (rabbit reticulocyte or HeLa cell lysate) is an expensive endeavor. Further efforts to reconstitute the mammalian multi-subunit factors from individual recombinant proteins (Sun et al., 2011; Suragani et al., 2006) can help overcome this hurdle and a detailed mechanistic understanding of mammalian translation initiation can be achieved by using fluorescencelabeled recombinant elFs/RNA, employing the methods described in this work. 


\section{MATERIALS}

\section{Table 3. Chemicals}

\begin{tabular}{|c|c|}
\hline $\begin{array}{l}\text { 2-Mercaptoethanol } \\
\text { 4-(2-hydroxyethyl)-1-piperazineethane } \\
\text { sulfonic acid (HEPES) }\end{array}$ & $\begin{array}{l}\text { Sigma-Aldrich - Taufkirschen, Germany } \\
\text { Sigma-Aldrich - Taufkirschen, Germany }\end{array}$ \\
\hline Acetic acid & Merck KGaA - Darmstatdt, Germany \\
\hline Acetonitrile & Merck KGaA - Darmstatdt, Germany \\
\hline Acrylamide (29:1) 40\% & Serva - Heidelberg, Germany \\
\hline Agar & BD - Le Pont de Claix, France \\
\hline Agarose & Serva - Heidelberg, Germany \\
\hline Aluminium chloride $\left(\mathrm{AlCl}_{3}\right)$ & Sigma-Aldrich - Taufkirschen, Germany \\
\hline Ammonium chloride $\left(\mathrm{NH}_{4} \mathrm{Cl}\right)$ & Merck KGaA - Darmstatdt, Germany \\
\hline Ammonium persulphate (APS) & Merck KGaA - Darmstatdt, Germany \\
\hline Ampicillin & Sigma-Aldrich - Taufkirschen, Germany \\
\hline Beryllium chloride $\left(\mathrm{BeCl}_{2}\right)$ & Sigma-Aldrich - Taufkirschen, Germany \\
\hline Boric acid & Merck KGaA - Darmstatdt, Germany \\
\hline Bovine serum albumin (BSA) & Sigma - Steinheim, Germany \\
\hline Complete EDTA free protease inhibitor & Roche - Indianapolis, USA \\
\hline Coomassie blue & Merck KGaA - Darmstatdt, Germany \\
\hline Dimethyl sulfoxide (DMSO) & Merck KGaA - Darmstatdt, Germany \\
\hline Ethanol & Merck KGaA - Darmstatdt, Germany \\
\hline Ethylenediamine tetraacetic acid (EDTA) & Merck KGaA - Darmstatdt, Germany \\
\hline Formic acid & Merck KGaA - Darmstatdt, Germany \\
\hline Glacial acetic acid & Merck KGaA - Darmstatdt, Germany \\
\hline Glycerol & Merck KGaA - Darmstatdt, Germany \\
\hline Hydrochloric acid (HCl) & J.T Baker - Daventer, Netherlands \\
\hline Imidazole & Merck KGaA - Darmstatdt, Germany \\
\hline Isopropyl $\beta$-D-1-thiogalactopyranoside (IPTG) & Roth - Karlsruhe, Germany \\
\hline Kanamycin & Calbiochem - California, USA \\
\hline Scintillation cocktail Lumasafe plus & PerkinElmer - Massachusetts, USA \\
\hline Scintillation cocktail Quickszint 361 & Zinsser analytic - Maidenhead, U.K \\
\hline Magnesium chloride $\left(\mathrm{MgCl}_{2}\right)$ & Merck KGaA - Darmstatdt, Germany \\
\hline
\end{tabular}




\begin{tabular}{|l|l|}
\hline Millipore water & Millipore - Massechusetts, USA \\
N,N,N',N'-tetramethylethylenediamine & Sigma-Aldrich - Taufkirschen, Germany \\
(TEMED) & \\
Phenylmethylsulfonylfluoride (PMSF) & Sigma-Aldrich - Taufkirschen, Germany \\
Potassium chloride (KCl) & Roche - Indianapolis, USA \\
Potassium fluoride (KF) & Merck KGaA - Darmstatdt, Germany \\
Potassium hydroxide (KOH) & Sigma-Aldrich - Taufkirschen, Germany \\
Sodium chloride (NaCl) & Merck KGaA - Darmstatdt, Germany \\
Sodium dodecyl sulphate (SDS) & Merck KGaA - Darmstatdt, Germany \\
Sodium orthovanadate & Serva - Heidelberg, Germany \\
Sodium metavanadate & Sigma-Aldrich - Taufkirschen, Germany \\
Sucrose & Sigma-Aldrich - Taufkirschen, Germany \\
Trifluoro acetic acid (TFA) & Merck KGaA - Darmstatdt, Germany \\
Tris(hydroxymethyl)-aminomethane (Tris) & Merck KGaA - Darmstatdt, Germany \\
Tryptone & Merck KGaA - Darmstatdt, Germany \\
Yeast extract & BD - Le Pont de Claix, France \\
\hline
\end{tabular}

Table 4. Fluorophores and radioactive compounds

Alexa 488 maleimide (Alx488)

Alexa 555 maleimide (Alx555)

Atto 540Q maleimide (Atto540Q)

Bodipy FL succinimidyl ester (Bpy)

lodoacetamide fluorescein (5' IAF)

2'(3')-O-(N-methylanthraniloyl) (Mant)

${ }^{3}[\mathrm{H}]$ Methionine

${ }^{14}[\mathrm{C}]$ Phenylalanine
Life Technologies - Darmstadt, Germany Life Technologies - Darmstadt, Germany Life Technologies - Darmstadt, Germany Life Technologies - Darmstadt, Germany Life Technologies - Darmstadt, Germany Jena Biosciences - Jena, Germany Perkin Elmar - Massachusetts, USA Perkin Elmar - Massachusetts, USA

\section{Table 5. Nucleotides}

7- methyl guanosine

Deoxy- nucleotide triphosphate (dNTP)

Guanosine-5'-triphosphate (GTP)
Sigma-Aldrich - Taufkirschen, Germany NEB - Massachusetts, USA Jena Biosciences - Jena, Germany 


\begin{tabular}{|l|l|}
\hline Guanosine-5'-diphosphate (GDP) & Jena Biosciences - Jena, Germany \\
Guanosine 5'-O-[Y-thio]triphosphate & Jena Biosciences - Jena, Germany \\
(GTPyS) & Life Technologies - Darmstadt, Germany \\
Bodipy-GTP & Life Technologies - Darmstadt, Germany \\
Bodipy- GDP & Life Technologies - Darmstadt, Germany \\
Bodipy- GTPyS & Jena Biosciences - Jena, Germany \\
Mant-GTP & Jena Biosciences - Jena, Germany \\
Mant-GTPYS & \\
\hline
\end{tabular}

Table 6. Kits

\begin{tabular}{|l|l|}
\hline Bradfords' assay kit & BIORAD - California, USA \\
Nucleospin plasmid isolation kit & Macherey Nagel - Dueren, Germany \\
Phusion DNA polymerase kit & NEB - Massachusetts, USA \\
\hline
\end{tabular}

\section{Table 7. Plasmids}

\begin{tabular}{|l|l|}
\hline pET11a - infA & Dept. Rodnina \\
pETM11- infB & Dept. Rodnina \\
pET28a - infC & Dept. Rodnina \\
pet24a - rpsM & Dept. Rodnina \\
pet24a - rpmG & Dept. Rodnina \\
\hline
\end{tabular}

\section{Table 8. DNA primers}

Truncation of infB (IF2 $\Delta 1-294)$

Truncation of infB by site-directed 5' CTG CAG CAA GGC TTC CAG AAG 3'

5' CAT GGC GCC CTG AAA ATA AAG ATT CTC AGT AGT GG 3' mutagenesis (IF2 $\Delta 791-890$ ) 5' CTC CGG AAC TGA AAC AGT AAA TTA TCG GTC TGG CGG AAG 3' 5' CTT CCG CCA GAC CGA TAA TTT ACT GTT TCA GTT CCG GAG 3' 
Table 9. mRNA

\begin{tabular}{|l|l|}
\hline 022 & 5`GGG AAU UCA AAA AUU UAA AAG \\
UUA ACA GGU AUA CAU ACU AUG \\
UUU ACG AUU ACU ACG AUC UUC \\
UUC ACU UAA UGC GUC UGC AGG \\
CAU GCA AGC U 3’ \\
\hline
\end{tabular}

Table 10. Enzymes

\begin{tabular}{|l|l|}
\hline DNAse & Sigma-Aldrich - Taufkirschen, Germany \\
Dpn1 & NEB - Frankfurt, Germany \\
Lysozyme & Sigma-Aldrich - Taufkirschen, Germany \\
Phusion DNA polymerase & Finnymes - Espoo, Finland \\
PNPase & Dept. Rodnina \\
Pyruvate kinase (PK) & Roche - Indianapolis, USA \\
T7 RNA polymerase & Dept. Rodnina \\
\hline
\end{tabular}

\section{Table 11. Buffers and solutions}

\begin{tabular}{|c|c|}
\hline \multirow[t]{2}{*}{ Buffer A for Ni-NTA } & 25 mM HEPES (pH 7.5) \\
\hline & 500 mM NaCl. \\
\hline \multirow[t]{4}{*}{ Buffer B for Ni-NTA } & 25 mM HEPES (pH 7.5) \\
\hline & $300 \mathrm{mM} \mathrm{NaCl}$ \\
\hline & 6 mM 2-mercaptoethanol \\
\hline & $5 \%$ glycerol \\
\hline \multirow[t]{5}{*}{ Buffer C for Ni-NTA } & 25 mM HEPES (pH 7.5) \\
\hline & $300 \mathrm{mM} \mathrm{NaCl}$ \\
\hline & 6 mM 2-mercaptoethanol \\
\hline & $5 \%$ glycerol \\
\hline & 10 mM imidazole \\
\hline \multirow[t]{3}{*}{ Buffer D for Ni-NTA } & 25 mM HEPES (pH 7.5) \\
\hline & $300 \mathrm{mM} \mathrm{NaCl}$ \\
\hline & 6 mM 2-mercaptoethanol \\
\hline
\end{tabular}




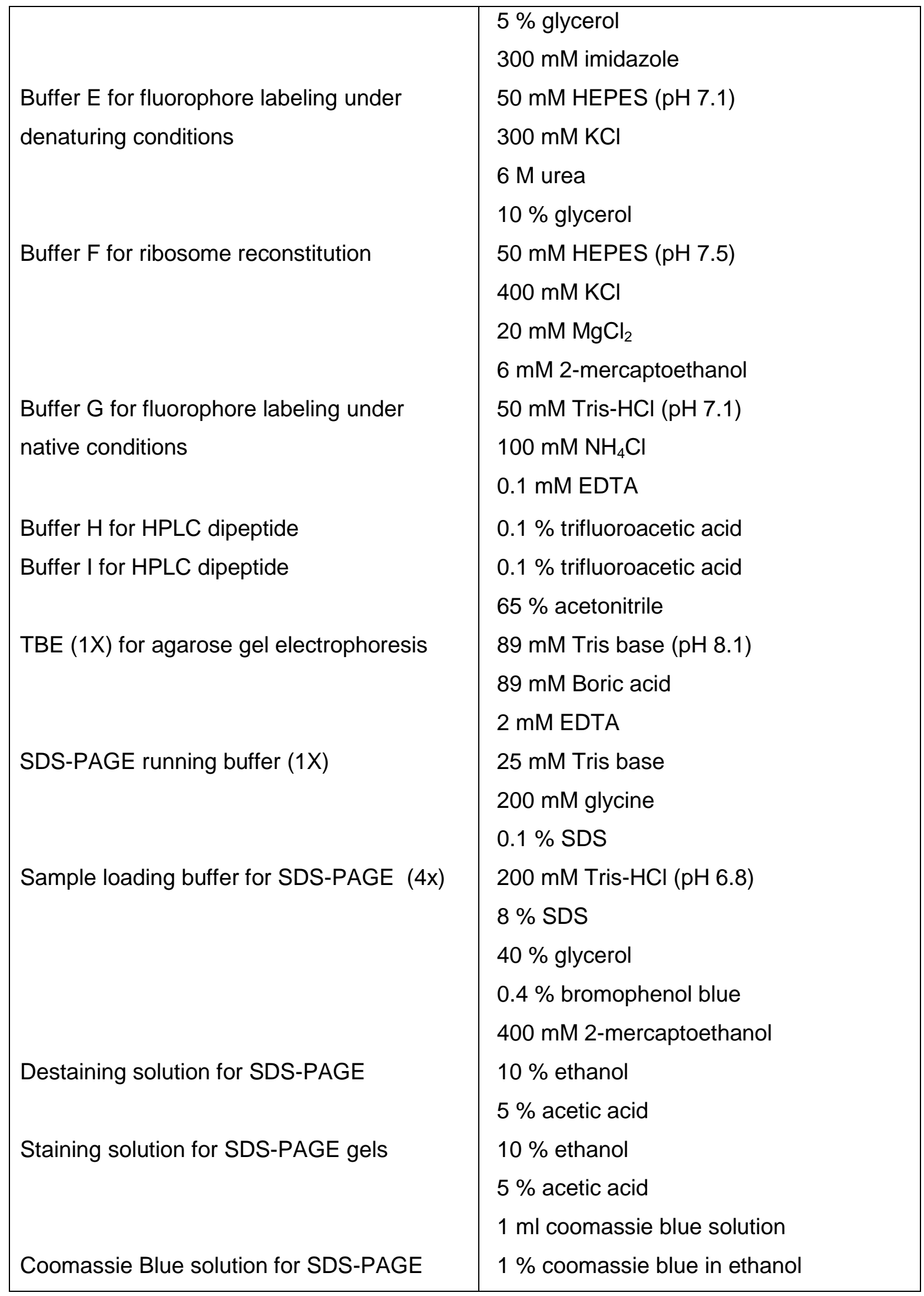




\begin{tabular}{|c|c|}
\hline \multirow[t]{2}{*}{ Pi-MOP mixture } & $0.1 \mathrm{U} / \mathrm{ml}$ PNPase \\
\hline & $200 \mu \mathrm{M}$ 7-methylguanosine \\
\hline \multirow[t]{4}{*}{ Lysis buffer } & 25 mM HEPES (pH 7.5) \\
\hline & $100 \mathrm{mM} \mathrm{NaCl}$ \\
\hline & $10 \mathrm{mM} \mathrm{MgCl}_{2}$ \\
\hline & 6 mM 2-mercaptoethanol \\
\hline \multirow[t]{5}{*}{ Protein Storage buffer } & 50 mM HEPES (pH 7.5) \\
\hline & $70 \mathrm{mM} \mathrm{NH}_{4} \mathrm{Cl}$ \\
\hline & $30 \mathrm{mM} \mathrm{KCl}$ \\
\hline & $7 \mathrm{mM} \mathrm{MgCl}_{2}$ \\
\hline & $10 \%$ glycerol \\
\hline \multirow[t]{4}{*}{$\mathrm{TAKM}_{7}$} & 50 mM Tris-HCl (pH 7.5) \\
\hline & $70 \mathrm{mM} \mathrm{NH}_{4} \mathrm{Cl}$ \\
\hline & $30 \mathrm{mM} \mathrm{KCl}$ \\
\hline & $7 \mathrm{mM} \mathrm{MgCl}_{2}$ \\
\hline
\end{tabular}

\section{Table 12. Cell strains}
E. coli BL21
E. coli MRE600
E. coli $12 \Delta S 13$
E. coli $\mathrm{K} 12 \Delta \mathrm{L} 33$

\section{Table 13. Cell culture media}

\begin{tabular}{|l|l|}
\hline LB broth & $10 \mathrm{~g} / \mathrm{l} \mathrm{NaCl}$ \\
& $10 \mathrm{~g} / \mathrm{l}$ tryptone \\
& $5 \mathrm{~g} / \mathrm{l}$ yeast extract \\
& $10 \mathrm{~g} / \mathrm{l} \mathrm{NaCl}$ \\
& $10 \mathrm{~g} / \mathrm{l}$ tryptone \\
& $5 \mathrm{~g} / \mathrm{l}$ yeast extract \\
& $15 \mathrm{~g} / \mathrm{l}$ agar \\
\hline
\end{tabular}


Table 14. Chromatographic columns

\begin{tabular}{|l|l|}
\hline RP-8 & Merck KGaA - Darmstatdt, Germany \\
Ni-NTA & Qiagen - Hilden, Germany \\
Hi-Trap HP SP & GE Healthcare - Uppsala, Sweden \\
\hline
\end{tabular}

Table 15. Instruments

\begin{tabular}{|l|l|}
\hline Cell density meter- Ultrospec 10 & GE Healthcare - Uppsala, Sweden \\
Cell power supply (Mini PROTEAN Tetra) & BIORAD - California, USA \\
Centrifuge & Beckmann Coulter - California, USA \\
Electrophoresis chamber & BIORAD - California, USA \\
Liquid scintillation counter & PerkinEImer - Massachusetts, USA \\
Quench-flow & Kintek - Texas, USA \\
Rotor JA 25.50 & Beckmann Coulter - California, USA \\
Rotor TLS-55 & Beckmann Coulter - California, USA \\
Rotor JLA 8.1000 & Beckmann Coulter - California, USA \\
Shaking incubator & Eppendorf - Hamburg, Germany \\
Stopped-flow & Applied Photophysics - Leatherhead, UK \\
Ultracentrifuge & Beckmann Coulter - California, USA \\
\hline
\end{tabular}

Table 16. Other materials

\begin{tabular}{|l|l}
\hline Centrifugal filter units & Amicon ultra - Carrigtwohill, Ireland \\
Dialysis cellulose membrane & SpectrumLab - Breda, Netherlands \\
DNA marker smartladder & Eurogentec - Belgium \\
Nitrocellulose membrane & Whatman - Kent, UK \\
Perfect protein marker 15-150 kDa & Novagen - San Diego, USA \\
Ultracentrifuge tubes (2 ml) & Beckman ultraclear - California, USA \\
Stopped-flow cut-off filters KV408, & Schott AG - Mainz, Germany \\
KV450, KV500, KV590 &
\end{tabular}


Table 17. Software

\begin{tabular}{|l|l|}
\hline Prism & GraphPad - California, USA \\
Kintek Explorer & Kintek - Texas, USA \\
Multigauge & Fujifilm - Tokyo, Japan \\
Prodata viewer & Applied Photophysics - Leatherhead, UK \\
\hline
\end{tabular}




\section{METHODS}

\subsection{Basic molecular biology techniques}

\subsubsection{Polymerase chain reaction (PCR)}

A reaction containing template DNA $(0.5 \mathrm{ng} / \mu \mathrm{l})$, primers $(0.5 \mu \mathrm{M})$, 1x Phusion polymerase HF buffer, dNTPs $(0.2 \mathrm{mM})$, DMSO (3\%), and Phusion Polymerase (0.2 unit/ $\mu \mathrm{l})$ was subjected to 30 cycles of PCR (Mullis et al., 1986) in a thermocycler.

Table 18. Conditions used for standard PCR reaction.

$\left.\begin{array}{|lll|}\hline \text { Initial denaturation } & 95^{\circ} \mathrm{C} & 2 \mathrm{~min} \\ \text { Denaturation } & 95^{\circ} \mathrm{C} & 30 \mathrm{~s} \\ \text { Annealing } & 55^{\circ} \mathrm{C} & 30 \mathrm{~s} \\ \text { Elongation } & 72^{\circ} \mathrm{C} & 30 \mathrm{~s} \\ \text { Final extension } & 72^{\circ} \mathrm{C} & 10 \mathrm{~min}\end{array}\right\} 30$ cycles

$5 \mu \mathrm{l}$ of the PCR product was analyzed by agarose gel (1\%) electrophoresis at $100 \mathrm{~V}$ for 40 min in 1X TBE buffer.

Site-directed mutagenesis of genes encoded into plasmids was performed in a $50 \mu \mathrm{l}$ reaction using methods described in the Quick-change site-directed mutagenesis kit (Stratagene California, USA).

Table 19. PCR conditions used for Site-directed mutagenesis

$\left.\begin{array}{|lll|}\hline \text { Initial denaturation } & 95^{\circ} \mathrm{C} & 5 \mathrm{~min} \\ \text { Denaturation } & 95^{\circ} \mathrm{C} & 1 \mathrm{~min} \\ \text { Annealing } & 55^{\circ} \mathrm{C} & 1 \mathrm{~min} \\ \text { Elongation } & 72^{\circ} \mathrm{C} & 4 \mathrm{~min}\end{array}\right\} \quad 18 \mathrm{cycles}$

Thereafter, 20 units of $D p n 1$ was added and the reaction was incubated for $2 \mathrm{~h}$ at $37^{\circ} \mathrm{C}$ in order to digest the methylated template DNA. 


\subsubsection{Transformation and plasmid isolation}

$2 \mu \mathrm{l}$ of the plasmid was added to $100 \mu \mathrm{l}$. coli BL21 chemically competent cells. The cells were incubated on ice for 15 mins, heat shocked at $42^{\circ} \mathrm{C}$ for $30 \mathrm{~s}$ and cooled on ice for 2 min. $400 \mu \mathrm{l}$ of LB broth was added and the mixture was incubated for 40 mins at $37^{\circ} \mathrm{C}$ in a shaking incubator. $100 \mu \mathrm{l}$ of the mixture was plated on LB agar plates containing $30 \mu \mathrm{g} / \mathrm{ml}$ kanamycin or $50 \mu \mathrm{g} / \mathrm{ml}$ amplicillin (depending on the resistance marker within the plasmid). The plates were incubated overnight at $37^{\circ} \mathrm{C}$. Next day, a single colony was picked and immersed in $5 \mathrm{ml} \mathrm{LB}$ broth containing antibiotic. The mixture was kept shaking at $37^{\circ} \mathrm{C}$ over day. The cultures were re-inoculated $(1: 100 \mathrm{v} / \mathrm{v})$ in a larger volume $(200 \mathrm{ml})$ of LB broth containing antibiotic and incubated at $37^{\circ} \mathrm{C}$ on a shaking incubator overnight. The plasmid was purified from $2 \mathrm{ml}$ bacterial culture using a Nucleospin plasmid isolation kit. The gene sequence as well as the presence of the desired mutation was confirmed by DNA sequencing (Peqlab - Erlangen, Germany).

\subsubsection{Protein expression and purification}

3 liters LB broth (containing antibiotic) was inoculated with the overnight culture to a final optical density of $0.1 \mathrm{~A}_{600}$. Protein expression was induced using $0.5 \mathrm{mM}$ IPTG when $\mathrm{A}_{600}$ of cells reached 0.8-0.9. Cells were harvested by centrifugation at $6000 \mathrm{rpm}$ in a Beckman Coulter JLA 8.1000 rotor for $20 \mathrm{~min}$. The cell pellet was dissolved in $2 \mathrm{ml}$ cell lysis buffer per gram of cells. $0.1 \mathrm{mM}$ PMSF and a protease inhibitor tablet were added and the cells were lysed with $1 \mathrm{mg} / \mathrm{ml}$ lysozyme. $5 \mathrm{\mu g} / \mathrm{ml}$ DNAse was added to the mixture to digest genomic DNA.

The cell lysate was centrifuged at 25,000 rpm for 30 mins using a Beckmann Coulter rotor JA 25.50. For His-tag bearing proteins, the supernatant was added to a $2 \mathrm{ml} \mathrm{Ni-NTA}$ column (Crowe et al., 1994) equilibrated with buffer $A$. The column was washed with two column volumes of buffer $B$ and thereafter with buffer $C$. The protein was eluted using buffer $D$ containing $300 \mathrm{mM}$ imidazole. Protein fractions eluted from the Ni-NTA column as well as those proteins lacking His-tags were purified by FPLC (Fast protein liquid chromatography) using previously published procedures (as indicated below). $10 \mu \mathrm{l}$ of samples collected from each purification step were loaded onto an SDS-PAGE gel together with a 15-150 kDa protein marker and run at $100 \mathrm{~V}$ for $2 \mathrm{~h}$ to visualize protein yield and purity. The purified protein samples were dialyzed into storage buffer using cellulose membrane. The protein was then concentrated using centrifugal units with appropriate cut-off filters. The protein was aliquoted, flash frozen in liquid nitrogen and stored at $-80^{\circ} \mathrm{C}$. Protein concentration was 
determined by Bradford's assay (Bradford, 1976) or SDS-PAGE using increasing amounts of BSA as a protein standard.

\subsubsection{SDS-PAGE}

Table 20. Preparation of SDS gels

\begin{tabular}{|l|l|l|}
\hline \multicolumn{3}{|l|}{ Resolving gel $10 \% 5 \mathrm{ml}$} \\
\hline $40 \%$ acrylamide $(29: 1)$ & 1.25 & $\mathrm{ml}$ \\
$1.5 \mathrm{M}$ Tris- $\mathrm{HCl} \mathrm{pH} 8.8$ & 1.25 & $\mathrm{ml}$ \\
water & 2.5 & $\mathrm{ml}$ \\
$10 \%$ SDS & 50 & $\mu \mathrm{l}$ \\
$10 \%$ APS & 50 & $\mu \mathrm{l}$ \\
TEMED & 5 & $\mu \mathrm{l}$ \\
\hline
\end{tabular}

\begin{tabular}{|l|l|l|}
\hline \multicolumn{3}{|l|}{ Stacking gel $4.4 \% 2 \mathrm{ml}$} \\
\hline $40 \%$ acrylamide (29:1) & 0.25 & $\mathrm{ml}$ \\
$1 \mathrm{M}$ Tris pH 6.8 & 0.25 & $\mathrm{ml}$ \\
water & 1.5 & $\mathrm{ml}$ \\
$10 \%$ SDS & 20 & $\mu \mathrm{l}$ \\
$10 \%$ APS & 20 & $\mu \mathrm{l}$ \\
TEMED & 2 & $\mu \mathrm{l}$ \\
\hline
\end{tabular}

4x SDS-PAGE loading dye was added to protein samples and heated at $95^{\circ} \mathrm{C}$ to denature proteins. $10 \mu \mathrm{l}$ of each sample was loaded onto a $10 \%$ SDS-PAGE gel and electrophoresis was performed in $1 \mathrm{x}$ running buffer at $150 \mathrm{~V}$ (Shapiro et al., 1967). The gel was washed with distilled water, $30 \mathrm{ml}$ of staining solution was added and incubated at room temperature for 4 h. The staining solution was discarded and $40 \mathrm{ml}$ destaining solution was added to the gel and incubated overnight on a shaking incubator at room temperature.

\subsection{Preparation of purified translation initiation components}

\subsubsection{Purification of non-fluorescent components}

The below mentioned components were kindly purified by our laboratory technicians- Sandra Kappler (ribosomes and EF-Tu), Olaf Geitzner (fMet-tRNA ${ }^{f M e t}$, Phe-tRNA ${ }^{\text {Phe }}$ ), Christina Kothe (IFs) and Tanja Wiles (mRNA).

$30 S$ and $50 S$ ribosomal subunits were prepared by zonal centrifugation from 705 ribosomes purified from E. coli MRE600 cells (Milon et al., 2007; Rodnina and Wintermeyer, 1995). Plasmids containing genes encoding the three IFs (IF1 (infA-pet11a), IF2 (infB-petM11) and IF3 (infC-pet28a)) were transformed into $E$. coli BL21 cells. IFs were over-expressed and purified using procedures described (Milon et al., 2007). Truncated mutant of IF2 ( $\Delta 1-294$ (Moreno et al., 1999)) was prepared by PCR-amplification of parts of the infB gene and cloning into the plasmid petM11. Truncated mutant of IF2 ( $\Delta 792-890$ (Mortensen et al., 1998)) was prepared by introducing a premature stop codon in the plasmid infB-petM11 
using site-directed mutagenesis. The truncated mutants were over-expressed in E. coli BL21 cells and purified using Ni-NTA affinity purification as described above. Recombinant EF-Tu was prepared as described (Milon et al., 2007; Rodnina et al., 1994). fMet-tRNA ${ }^{\text {fMet }}$ was purified by HPLC (Milon et al., 2007) and was 95\% aminoacylated and formylated. 022 mRNA (94 bp) was prepared by T7 RNA polymerase transcription (Milon et al., 2007).

\subsubsection{Preparation of fluorescence-labeled components}

In general, fluorescence labeling of recombinant proteins was performed by mutagenesis of a single, non-conserved, solvent-exposed residue to cysteine, and subsequently labeling the introduced cysteine residue with a thiol-reactive fluorophore (Alexa488, Alexa555 and Atto540Q) using maleamide chemistry. Alexa and Atto describe the name of fluorescent probe, while the adjacent number indicates the maximum excitation wavelength of the dye. To prevent labeling of the protein at multiple positions, the native cysteine residues at the surface of the protein were mutated to non-reactive serine residues.

\subsubsection{1. $30 \mathrm{~S}$ and $50 \mathrm{~S}$ subunits}

$30 S$ subunits which lacked the ribosomal protein $S 13$ (30S $\Delta S 13$ ) or $50 S$ subunits lacking the ribosomal protein L33 (50S $\Delta \mathrm{L} 33$ ) were expressed from E. coli strain K12 (kindly provided by Prof. Rachel Green (Johns Hopkins University) and Prof. Janine Maddock (University of Michigan), respectively). $30 \mathrm{~S} \Delta \mathrm{S} 13$ and $50 \mathrm{~S} \Delta \mathrm{L} 33$ ribosomal subunits were purified as previously described (Milon et al., 2007) and subsequently reconstituted with fluorescencelabeled ribosomal protein S13 and L33, respectively (see below).

For fluorescence labeling of $\mathrm{S} 13$ (encoded by gene rpsM cloned in plasmid pET24a) and L33 (encoded by gene rpmG cloned in plasmid pET24a), cysteine residues were introduced at positions 112 and 31, respectively. To prevent double-labeling of protein, the native cysteine residue of $\mathrm{S} 13$ (Cys85) was mutated to serine. The proteins were over-expressed in E. coli BL21 cells and subsequently purified according to procedures described (Hickerson et al., 2005). Labeling of recombinant S13 and L33 with fluorescent dye (Alexa488) or quencher dye (Atto540Q) was performed under denaturing conditions with a 10-fold molar excess of dye in buffer $\mathrm{E}$. The reaction mix was incubated for $2 \mathrm{~h}$ at $25^{\circ} \mathrm{C}$ and stopped by adding 6 $\mathrm{mM}$ 2-mercaptoethanol. The excess dye was removed using a cation exchange HiTrap SP HP column and the proteins were refolded (Hickerson et al., 2005). The efficiency of labeling was assessed by SDS-PAGE and spectrophotometric analysis and was 100\%. Finally, $30 S \Delta$ S13 and 50SA L33 subunits were reconstituted with 1.5-fold molar excess of 
fluorescence-labeled S13 and L33 ribosomal proteins, respectively, in buffer F. The reaction mix was incubated in the dark for $1 \mathrm{~h}$ at $47^{\circ} \mathrm{C}$. The excess fluorescent proteins were removed by loading the samples on a $30 \%$ sucrose cushion prepared using buffer $F$. The ribosomal pellets were suspended in $\mathrm{TAKM}_{7}$ buffer and the efficiency of reconstitution with fluorescent ribosomal proteins was determined as $\sim 90-100 \%$. The fluorescent subunits were flash frozen and stored at $-80^{\circ} \mathrm{C}$. The procedure was established in our laboratory and performed by $\mathrm{Dr}$. Riccardo Belardinelli.

\subsubsection{Initiation factors}

Cysteine residues were engineered at position 4 in IF1, position 757 in IF2 and position 166 in IF3 to enable fluorescent labeling. At the same time, the native cysteine residues in IF2 (Cys599) and IF3 (Cys65) were replaced by serine to prevent double-labeling of the protein. The plasmids were transformed into E. coli strain BL21 and all IFs were over-expressed and purified according to procedures described (Milon et al., 2007). Fluorescent labeling of IFs was also performed essentially as described (Milon et al., 2007). Briefly, the purified IFs were dialyzed into labeling buffer $G$ and subsequently labeled with a 5 -fold molar excess of fluorescent dyes (Alexa488 or Alexa555) or quencher dye (Atto540Q) over the protein for $2 \mathrm{~h}$ at $25^{\circ} \mathrm{C}$ (Milon et al., 2007). The reaction was performed in the dark and stopped by adding a 10-fold molar excess of 2-mercaptoethanol. The excess dye was removed using a cation exchange HiTrap SP HP column. The efficiency of labeling was assessed by SDS-PAGE and spectrophotometric analysis and was $>90 \%$. The procedure was established in our laboratory by Dr. Pohl Milon. The mutant IFs were purified by Christina Kothe. The fluorescence-labeling was performed by Dr. Riccardo Belardinelli, Dr. Pohl Milon and Dr. Cristina Maracci.

\subsubsection{3. $\quad$ tRNA $^{\text {fMet }}$}

$\mathrm{tRNA}^{\mathrm{fMet}}$ was labeled at a modified base (thio-uridine) at position 8 with an iodoacetamide derivative of the fluorescent dye, fluorescein (5'-IAF), according to protocols described (Milon et al., 2007). The labeling reaction was stopped by adding 0.3 M KAc (pH 5.0) and the excess dye was removed by four rounds of ethanol precipitation. The efficiency of labeling was assessed by spectrophotometric analysis and scintillation counting of the associated radioactivity, and was $100 \%$. The fluorescein-labeled tRNA $^{\mathrm{fMet}}(\mathrm{Flu})$ was subsequently aminoacylated with radioactive ${ }^{3}[\mathrm{H}]$ Met, formylated and purified according to detailed procedures (Milon et al., 2007). The procedure was performed by Olaf Geitzner. 
Unformylated and purified ${ }^{3}[\mathrm{H}]$ Met-tRNA $A^{\text {fMet }}$ was labeled with Bodipy-FL sulfosuccinimidyl ester at the amino group of Met to form Bpy- ${ }^{3}[\mathrm{H}]$ Met-tRNA ${ }^{\text {fMet }}$. The procedures for labeling have been previously described (Holtkamp et al., 2014). The labeling reaction was stopped by adding $0.2 \mathrm{M} \mathrm{KAc}(\mathrm{pH} \mathrm{5.0)}$ ) and the excess dye was removed by four rounds of ethanol precipitation. The concentration of labeled $\left.\mathrm{Bpy}^{3}{ }^{3} \mathrm{H}\right] \mathrm{Met}-\mathrm{tRNA}{ }^{\mathrm{fMet}}$ was determined by spectrophotometric analysis and the concentration of total ${ }^{3}[\mathrm{H}]$ Met-tRNA ${ }^{\mathrm{fMet}}$ was determined by radioactive counting. In this way, the efficiency of labeling was determined as $\sim 80 \%$. The procedure was established in our laboratory by Dr. Wolf Holtkamp and performed by Olaf Geitzner and Irena Andreeva.

\subsection{Biochemical methods}

\subsubsection{S IC formation}

30S subunits were activated in $\mathrm{TAKM}_{7}$ buffer containing an additional $14 \mathrm{mM} \mathrm{MgCl}$ for 30 $\min$ at $37^{\circ} \mathrm{C}$. 30S IC was prepared by incubating $30 \mathrm{~S}$ subunits $(0.1-0.3 \mu \mathrm{M}$; the concentration varied depending on the particular experiment (see results section)) with a 2-fold molar excess of the three IFs and a 5 -fold molar excess of $f^{3}[H] M e t-t R N A^{\text {fMet }}$ and 022 mRNA in $\mathrm{TAKM}_{7}$ buffer containing $0.25 \mathrm{mM}$ GTP. The reaction mix was incubated at $37^{\circ} \mathrm{C}$ for $30 \mathrm{~min}$ to form 30 S IC and thereafter kept on ice to stabilize the complexes.

\subsubsection{Purification of $70 S$ IC (containing Bpy-Met-tRNA ${ }^{\text {fMet }}$ )}

70 IC (Bpy) were prepared by incubating 70 S ribosomes $(0.5 \mu \mathrm{M})$ with IF1 $(1 \mu \mathrm{M})$, IF2 (1 $\mu \mathrm{M})$, IF3 $(1 \mu \mathrm{M}), \mathrm{Bpy}^{3}{ }^{3}[\mathrm{H}] \mathrm{Met}-\mathrm{tRNA} \mathrm{f}^{\mathrm{fMet}}(1 \mu \mathrm{M})$ and $022 \mathrm{mRNA}(1.5 \mu \mathrm{M})$ in $3 \mathrm{ml} \mathrm{TAKM}$ buffer containing GTP $(0.5 \mathrm{mM})$. The reaction mix was incubated at $37^{\circ} \mathrm{C}$ for $1 \mathrm{~h}$ to form $70 \mathrm{~S} \mathrm{IC}$ and thereafter kept on ice to stabilize the complexes. $600 \mu \mathrm{l}$ of filtered $1.1 \mathrm{M}$ sucrose solution, prepared in $\mathrm{TAKM}_{7}$ buffer, was added to $2 \mathrm{ml}$ ultracentrifuge tubes used in Beckman Coulter TLS-55 rotor. $1.4 \mathrm{ml}$ of the 70 IC (Bpy) reaction mix was layered on top of the sucrose solution and centrifuged for $4 \mathrm{~h}$ at 55,000 rpm. The pellet, containing 70S IC (Bpy), was dissolved in $150 \mu \mathrm{L} \mathrm{TAKM} M_{7}$ buffer and flash frozen and stored at $-80^{\circ} \mathrm{C}$. The concentration of Bpy- $^{3}[\mathrm{H}]$ Met-tRNA ${ }^{\text {fMet }}$ was determined by radioactive counting and the concentration of $70 \mathrm{~S}$ ribosome was determined by measuring the absorbance at $260 \mathrm{~nm}$. In this way, the efficiency of 70 S IC (Bpy) formation was determined as $\sim 85 \%$. 


\subsection{Rapid Kinetics Techniques}

\subsubsection{Stopped-Flow}

Stopped-flow is a technique used to study pre-steady state reaction kinetics occurring in msec-sec time range. The instrument records spectroscopic changes which take place after rapid mixing of two reactants. Often, one or two components in the reaction are fluorescent and change their fluorescence or FRET upon interaction. The change in fluorescence/FRET is recorded with time and provides information about the rate and extent of the reaction. Briefly, $60 \mu$ of each reactant solution is rapidly mixed together in a mixing chamber. The reaction mixture is then transferred to an observation cuvette where the emitted fluorescence passes through an appropriate cut-off filter and is detected by a photomultiplier mounted at a $90^{\circ}$ angle to the excitation beam. The flow of the reaction mixture into the cuvette is then stopped with the help of a stop syringe and the change in fluorescence is measured as a function of time using a SX-18MV stopped-flow apparatus.

1000 - 4000 time points are collected in a logarithmic time scale and the time courses are subsequently analyzed using exponential functions or numerical integration. Standard errors were calculated from fitting of the average derived from 7-10 time courses for each reaction. The dead-time of the instrument is about $1 \mathrm{~ms}$ and thus reactions occurring before this time cannot be monitored.

\subsubsection{Quench-Flow}

The quench-flow technique is used to study reaction kinetics occurring in msec-sec time range. $14 \mu \mathrm{l}$ of each reactant is rapidly mixed together and the reaction is stopped after a specified period of time using a chemical quench solution such as formic acid or $\mathrm{KOH}$. The quenched sample is collected and the product is separated from the reactants using chromatography. The extent of product formation is quantified by measuring the radioactivity associated with the reactant and the product. The time course of the reaction can be determined by measuring the product formation after quenching the reaction at different time points. The dead-time of the instrument is about $3.5 \mathrm{~ms}$ and thus reactions occurring before this time cannot be monitored. Hence, a quench-flow machine can be conveniently used to quench reactions between $0.004-20 \mathrm{~s}$. For longer time periods it is more convenient to quench the reaction by hand. 


\subsection{Rapid kinetic experiments}

30S IC was prepared as described previously. Variations in the protocol (for example, using a different concentration of initiation component or replacing a non-fluorescent component with its fluorescent counterpart) were made as indicated. All experiments were carried out in $\mathrm{TAKM}_{7}$ buffer at $20^{\circ} \mathrm{C}$, unless indicated otherwise. For use in the stopped-flow apparatus, $700 \mu \mathrm{l}$ of each reactant mix was prepared. For use in the quench-flow machine, $350 \mu \mathrm{l}$ of each reactant mix was prepared to measure at least 15 time points. The concentrations indicated in the methods section are those in the reactant mix. Because equal volumes of the two reactants were mixed together, the final concentration of each component (as reported in the results section), is half of what is indicated here.

\subsubsection{Subunit association}

50S subunit joining to 30 S IC was monitored by light scattering (LS) using the stopped-flow apparatus (Antoun et al., 2004; Grunberg-Manago et al., 1975). Briefly, equal volumes of 30S IC $(0.1-0.3 \mu \mathrm{M})$ and at least a 3-fold molar excess of 50 S subunits were rapidly mixed together and the light scattered perpendicular to the excitation beam of light $(434 \mathrm{~nm})$ was monitored in the absence of a cut-off filter. When the two subunits associated with each other to form a $70 S$ complex, an increase in LS was observed.

\subsubsection{Nucleotide interaction with IF2}

The interaction of GTP with the G-domain of IF2 was monitored using the fluorescent GTP analogs mant-GTP and Bpy-GTP. GTPase activation and dissociation of IF2 from the 70S IC were monitored via FRET changes between mant-GTP and the intrinsic Trp residue of the factor (Fabbretti et al., 2012; Milon et al., 2007). Direct excitation was at $290 \mathrm{~nm}$ and the output was monitored after passing through cut-off filter KV408. The reaction was monitored by rapidly mixing $30 \mathrm{~S} \mathrm{IC}(0.3 \mu \mathrm{M})$ formed with mant-GTP $(10 \mu \mathrm{M})$ with varying concentrations of 50 s subunits $(0.9-3 \mu \mathrm{M})$ in a stopped-flow apparatus.

Binding of Bpy-GTP and Bpy-GDP to IF2 was monitored by rapidly mixing free IF2 (0.2 $\mu \mathrm{M})$ or IF2 bound to $30 \mathrm{~S} \mathrm{IC}(0.2 \mu \mathrm{M})$, formed in the absence of any nucleotide, with Bpy-GTP or Bpy-GDP $(4 \mu \mathrm{M})$ in a stopped-flow apparatus. The dissociation of Bpy-GTP or Bpy-GDP from IF2 on the 30 S IC was monitored by rapidly mixing $30 S \mathrm{IC}(0.2 \mu \mathrm{M})$, formed in the presence of the fluorescent nucleotide $(4 \mu \mathrm{M})$, with non-fluorescent GTP $(0.5 \mathrm{mM})$. Dissociation of Bpy-GDP from IF2 after $50 \mathrm{~S}$ subunit joining and GTP hydrolysis was monitored by rapidly mixing $30 \mathrm{~S} \mathrm{IC}(0.2 \mu \mathrm{M})$, formed in the presence of $4 \mu \mathrm{M}$ Bpy-GTP, with varying 
concentrations of 50 S subunits (0.6-4 $\mu \mathrm{M})$ in a stopped-flow apparatus. Direct excitation of Bpy was at $470 \mathrm{~nm}$ and the output was monitored after passing through cut-off filter KV500.

\subsubsection{Pi release from IF2}

To monitor Pi release from IF2 after GTP hydrolysis, an indicator reaction was used where the fluorescent derivative of phosphate binding protein, MDCC-PBP, rapidly binds $\mathrm{Pi}$ after release. To remove any pre-existing inorganic phosphate $(\mathrm{Pi})$, the stopped-flow apparatus was incubated and washed with the Pi-MOP mixture (Brune et al., 1994) for $1 \mathrm{~h}$ at $37^{\circ} \mathrm{C}$. 30 SIC $(0.1 \mu \mathrm{M})$ formed in the presence of GTP $(20 \mu \mathrm{M})$ and varying concentrations of $50 \mathrm{~S}$ subunits $(0.3-2 \mu \mathrm{M})$, were incubated with fluorescent MDCC-PBP protein $(4 \mu \mathrm{M})$ and the PiMOP mixture for $10 \mathrm{~min}$ at $37^{\circ} \mathrm{C}$. The two reactants were rapidly mixed together in the stopped-flow machine and the Pi release from IF2 was monitored by the fluorescence change of MDCC-PBP upon Pi binding. Direct excitation of MDCC was at $425 \mathrm{~nm}$ and the output was monitored after passing through cut-off filter KV450.

\subsection{4. $\quad$ tRNA ${ }^{\text {fMet }}$ interaction with IF2}

The dynamics of the 3' end of tRNA ${ }^{\text {fMet }}$ were monitored using a Bodipy-FL label attached at the amino group of Met (Bpy-Met-tRNA ${ }^{\mathrm{fMet}}$ ) (Gite et al., 2000; Holtkamp et al., 2014). The position of the fluorophore allowed the monitoring of the direct interaction of the 3' CCA-end of Bpy-Met-tRNA ${ }^{\text {fMet }}$ with the C2-domain of IF2. Recruitment of Bpy-Met-tRNA ${ }^{\text {fMet }}$ to the 30S complex was studied by rapidly mixing $30 \mathrm{~S} \mathrm{IC} \mathrm{(formed} \mathrm{in} \mathrm{the} \mathrm{absence} \mathrm{of} \mathrm{fMet-tRNA}{ }^{\mathrm{fMet}}$ ) $(0.2$ $\mu \mathrm{M})$ with Bpy-Met-tRNA ${ }^{\text {fMet }}(0.6 \mu \mathrm{M})$ at $37^{\circ} \mathrm{C}$ in a stopped-flow apparatus. The dissociation of Bpy-Met-tRNA ${ }^{\text {fMet }}$ from the $30 \mathrm{~S}$ IC was studied by rapidly mixing $0.1 \mu \mathrm{M} 30 \mathrm{~S}$ IC (formed in the presence of Bpy-Met-tRNA $\left.{ }^{\mathrm{fMet}}(0.2 \mu \mathrm{M})\right)$ with fMet-tRNA ${ }^{\mathrm{fMet}}(2 \mu \mathrm{M})$ in a stopped-flow apparatus. Release of Bpy-Met-tRNA ${ }^{\text {fMet }}$ from IF2 after subunit joining was monitored by rapidly mixing $30 \mathrm{~S} \mathrm{IC}(0.1 \mu \mathrm{M})$, formed in the presence of Bpy-Met-tRNA $A^{\text {fMet }}(0.3 \mu \mathrm{M})$, with varying concentrations of $50 \mathrm{~S}$ subunits $(0.3-2 \mu \mathrm{M})$. Direct excitation of Bpy was at $470 \mathrm{~nm}$ and the output was monitored after passing through cut-off filter KV500.

\subsubsection{IF2 interaction with the $30 \mathrm{~S}$ subunit}

The binding of IF2 to 30 S IC was checked by rapidly mixing 30S PIC $(0.2 \mu \mathrm{M})$ lacking IF2 with IF2 ${ }_{757}$ (Alx555) $(0.1 \mu \mathrm{M})$, in a stopped-flow apparatus. The dissociation of IF2 from $30 \mathrm{~S}$ IC was monitored by rapidly mixing 30 S IC $(0.2 \mu \mathrm{M})$ containing IF2 $2_{757}(\mathrm{Al}$ (x55) $(0.1 \mu \mathrm{M})$ with non-fluorescent IF2 $(2 \mu \mathrm{M})$. To monitor the binding of IF2 to 30 S complex after the first round of initiation, $30 \mathrm{~S} \mathrm{IC}(0.2 \mu \mathrm{M})$ containing $\operatorname{IF} 2_{757}(\mathrm{Al} \times 555)(0.1 \mu \mathrm{M})$ was rapidly mixed with 
varying concentrations of 50 S subunits $(0.5-2 \mu \mathrm{M})$. Direct excitation of Alx555 was at 555 $\mathrm{nm}$ and the output was monitored after passing through cut-off filter KV590.

\subsubsection{IF1 dynamics}

IF1 dynamics on the ribosome were monitored with the help of two observables in the stopped-flow apparatus: (i) the fluorescence change of $\mathrm{IF}_{4}(\mathrm{Al} \times 555)$; direct excitation of Alx555 was at $555 \mathrm{~nm}$ and the output was monitored after passing through cut-off filter KV590 and (ii) the FRET between $30 \mathrm{~S}_{\mathrm{S} 13}$ (Alx488) and $\mathrm{IF} 1_{4}$ (Atto540Q); direct excitation of Alx488 was at $470 \mathrm{~nm}$ and the output was monitored after passing through cut-off filter KV500. In case of the latter observable, the increase in proximity between the two factors resulted in a decrease in fluorescence due to the fluorescence quenching of $30 \mathrm{~S}_{\mathrm{S} 13}$ (Alx488) by $\mathrm{IF}_{4}($ (Atto540Q). The fluorescence/FRET changes depicted by the two observables represent changes in IF1 environment on the ribosome and the binding/dissociation of the factor from the ribosome, respectively.

To study the binding of IF1 to the $30 \mathrm{~S} \mathrm{IC,} \mathrm{IF1} 1_{4}(\mathrm{Al} \times 555)$ or $\mathrm{IF} 1_{4}$ (Atto540Q) $(0.2 \mu \mathrm{M})$ was rapidly mixed with $30 \mathrm{~S} I \mathrm{IC}$ or $30 \mathrm{~S}_{\mathrm{S} 13}(\mathrm{Alx} 488)$ IC $(0.1 \mu \mathrm{M})$ lacking IF1, respectively. The dissociation of IF1 from the $30 \mathrm{~S}$ IC was monitored by rapidly mixing $30 \mathrm{~S}$ IC containing $\mathrm{IF}_{1}(\mathrm{Al} \times 555)$ or $30 \mathrm{~S}_{\mathrm{S} 13}(\mathrm{Al} \times 488)$ IC containing IF1 ${ }_{4}$ (Atto540Q) $(0.1 \mu \mathrm{M})$, with non-fluorescent IF1 $(2 \mu \mathrm{M})$. To study the dynamics of IF1 after 50 S subunit joining, $30 \mathrm{~S}$ IC containing $\mathrm{IF} 1_{4}(\mathrm{Al} \times 555)$ or $30 \mathrm{~S}_{\mathrm{S} 13}(\mathrm{Al} \times 488)$ IC containing IF1 ${ }_{4}($ Atto540Q) $(0.1 \mu \mathrm{M})$, was rapidly mixed with varying concentrations of $50 \mathrm{~S}$ subunits $(0.3-2 \mu \mathrm{M})$.

\subsubsection{IF3 dynamics}

The dynamics of IF3 on the ribosome was monitored via FRET between IF3 ${ }_{166}(\mathrm{Al} \times 488)$ and IF2 757 (Atto540Q) in the stopped-flow apparatus. Direct excitation of Alx488 was at $470 \mathrm{~nm}$ and the output was monitored after passing through cut-off filter KV500. The increase in proximity between the two factors resulted in a decrease of fluorescence due to the quenching of IF3 ${ }_{166}$ (Alx488) fluorescence by IF2 ${ }_{757}$ (Atto540Q). The binding of IF3 to 30 S IC was monitored by rapidly mixing IF3 ${ }_{166}$ (Alx488) $(0.2 \mu \mathrm{M})$ with $30 \mathrm{~S} \mathrm{IC} \mathrm{(lacking} \mathrm{IF3} \mathrm{and}$ containing IF2 ${ }_{757}$ (Atto540Q) instead of non-fluorescent IF2) $(0.1 \mu \mathrm{M})$. The dissociation of IF3 from the $30 \mathrm{~S} \mathrm{IC}$ was monitored by rapidly mixing $30 \mathrm{~S} \mathrm{IC}(0.1 \mu \mathrm{M})$, formed in the presence of IF2 $2_{757}$ (Atto540Q) and IF3 ${ }_{166}$ (Alx488), with non-fluorescent IF3 (2 $\left.\mu \mathrm{M}\right)$. To study the dynamics of IF3 after 50 S subunit joining, 30S IC $(0.1 \mu \mathrm{M})$ formed in the presence of IF2 ${ }_{757}$ (Atto540Q) 
and $\mathrm{IF} 3_{166}(\mathrm{Alx} 488)$ was rapidly mixed with varying concentrations of $50 \mathrm{~S}$ subunits $(0.3-2$ $\mu \mathrm{M})$.

\subsubsection{First peptide bond formation}

EF-Tu-GTP_Phe-tRNA ${ }^{\text {Phe }}$ ternary complex (EF-Tu TC) was prepared by incubating EF-Tu $(1.6 \mu \mathrm{M})$, phosphoenol pyruvate $(2 \mathrm{mM})$, pyruvate kinase $(0.1 \mu \mathrm{g} / \mu \mathrm{l}),\left[{ }^{14} \mathrm{C}\right] \mathrm{Phe}-\mathrm{tRNA}{ }^{\text {Phe }}(0.8$ $\mu \mathrm{M})$ and GTP $(0.5 \mathrm{mM})$ in $\mathrm{TAKM}_{7}$ buffer for $15 \mathrm{~min}$ at $37^{\circ} \mathrm{C}$ (Rodnina et al., 1994). Varying concentrations of $50 \mathrm{~S}$ subunits $(0.6-2 \mu \mathrm{M})$ were added to the ternary complex mix. To monitor the first peptide bond formation, 30S IC $(0.2 \mu \mathrm{M})$ was rapidly mixed with $50 \mathrm{~S}$ subunits and EF-Tu TC in a quench-flow machine and incubated for different times (0.01- 10 s). The reaction was quenched with $0.5 \mathrm{M} \mathrm{KOH}$. The samples were incubated for $30 \mathrm{~min}$ at $37^{\circ} \mathrm{C}$ to hydrolyze RNA and then neutralized with $1 / 10^{\text {th }}$ sample volume of acetic acid. The final volume was corrected to $200 \mu \mathrm{l}$ using $0.1 \%$ TFA. The samples were centrifuged for 10 min at $13000 \mathrm{rpm}$ to pellet debris and then loaded onto an HPLC reverse chromatography column RP-8. Free amino acids and dipeptides were eluted using a linear gradient of buffer $\mathrm{H}$ and buffer I. The flow rate was $2 \mathrm{ml} / \mathrm{min}$ and the eluate was collected in 17 fractions of $1 \mathrm{ml}$ each. $2 \mathrm{ml}$ Lumasafe Plus liquid scintillation cocktail was added to each vial and $\left[{ }^{3} \mathrm{H}\right]$ and $\left[{ }^{14} \mathrm{C}\right]$ radioactivity in the fractions was measured in a liquid scintillation counter.

\subsection{9. (Re)-association of IF1 and IF2 with mature 70S IC}

IF2 release from, and subsequent re-association with, 70S IC was measured by rapidly mixing 30S IC $(0.1 \mu \mathrm{M})$, formed in the presence of Bpy-Met-tRNA $A^{\mathrm{fMet}}(0.3 \mu \mathrm{M})$ and GTP $(25$ $\mu \mathrm{M})$, with 50 S subunits $(0.5 \mu \mathrm{M})$, IF2 $(8 \mu \mathrm{M})$ and GTPYS $(0.5 \mathrm{mM})$. Binding of IF2 to mature 70 S IC was measured by rapidly mixing purified 70 S IC (Bpy) $(20 \mathrm{nM})$ with IF2 $(8 \mu \mathrm{M})$ bound to different GTP analogs $(0.5 \mathrm{mM})$ such as GTP, GDP, GDPNP and GTPYS. The reaction was also monitored in the presence of GDP $(0.1 \mathrm{mM})$ along with different phosphate analogs (0.1 mM) such as $\mathrm{BeF}_{3}\left(5 \mathrm{mM} \mathrm{KF}\right.$ and $0.1 \mathrm{mM} \mathrm{BeCl}_{3}$ in $\mathrm{TAKM}_{7}$ buffer), $\mathrm{AlF}_{3}(5 \mathrm{mM} \mathrm{KF}$ and $0.1 \mathrm{mM} \mathrm{AlCl}_{3}$ in $\mathrm{TAKM}_{7}$ buffer), ortho- and meta-vanadate. The fluorescence changes of BpyMet-tRNA ${ }^{\text {fMet }}$ were followed with time.

IF1 release from, and subsequent re-association with, 70S IC was measured by rapidly mixing $30 \mathrm{~S}_{\mathrm{S} 13}(\mathrm{Al} \times 488) \mathrm{IC}(0.1 \mu \mathrm{M})$ containing IF1 ${ }_{4}$ (Atto540Q) $(0.2 \mu \mathrm{M})$ and GTP $(25 \mu \mathrm{M})$, with $50 \mathrm{~S}$ subunits $(0.5 \mu \mathrm{M})$ and GTPYS $(0.5 \mathrm{mM})$. The $30 \mathrm{~S}_{\mathrm{S} 13}(\mathrm{Al} \times 488) \mathrm{IC}$ was formed in the absence of IF3 to promote rapid subunit joining to all complexes, regardless of composition. To measure the binding of $\mathrm{IF}_{4}\left(\right.$ Atto540Q) to mature $70 \mathrm{~S}_{\mathrm{S13}}(\mathrm{Alx} 488) \mathrm{IC}, 30 \mathrm{~S}_{\mathrm{S} 13}(\mathrm{Al} \times 488) \mathrm{IC}$ 
$(0.1 \mu \mathrm{M})$ was formed in the presence of GTP $(12.5 \mu \mathrm{M})$ but in the absence of IF1 and IF3. $70 \mathrm{~S}_{\mathrm{S} 13}(\mathrm{Alx} 488) \mathrm{IC}$ was prepared by adding $50 \mathrm{~S}$ subunits $(0.15 \mu \mathrm{M})$ to $30 \mathrm{~S}_{\mathrm{S} 13}(\mathrm{Al} \times 488) \mathrm{IC}$ and incubating the reaction mix at $37^{\circ} \mathrm{C}$ for $10 \mathrm{~min}$. Unpurified $70 \mathrm{~S}_{\mathrm{S} 13}$ (Alx488) IC $(0.1 \mu \mathrm{M})$ was rapidly mixed with $\mathrm{IF}_{4}($ Atto540Q) $(0.2 \mu \mathrm{M})$ and GTP/GTPYS $(0.5 \mathrm{mM})$ in the stopped-flow apparatus.

\subsection{Kinetic data modeling}

To formulate a kinetic model for 70S IC formation, time courses for each observable were collectively evaluated by numerical integration using a 10-step model (see Results section). For Pi release and FRET between mant-GTP and IF2, the fit of the buffer control trace (obtained in the absence of $50 \mathrm{~S}$ subunits) was subtracted from the respective time courses obtained in the presence of $50 \mathrm{~S}$ subunits. All time courses which did not contain a prominent delay phase before the start of the reaction were evaluated using single- or doubleexponential equations to obtain apparent rates of the reaction. Where the time courses were fit with a double-exponential function, the second (minor phase), in most cases, resulted from compositional heterogeneity of the $30 \mathrm{~S}$ complexes and a portion of poorly-active $50 \mathrm{~S}$ subunits. Because the rate and amplitude of this phase varied with different preparations of initiation components, only the rate constant of the relevant (predominant) phase of the reaction is discussed in the Results section. Exponential fitting calculations were performed using Prism (Graphpad Software) and numerical integration calculations were performed using KinTek Explorer (KinTek corporation, USA). Standard errors were calculated from fitting of the average derived from 7-10 time courses. 


\section{REFERENCES}

Acker, M.G., Kolitz, S.E., Mitchell, S.F., Nanda, J.S., and Lorsch, J.R. (2007). Reconstitution of yeast translation initiation. Methods in enzymology 430, 111-145.

Acker, M.G., Shin, B.S., Nanda, J.S., Saini, A.K., Dever, T.E., and Lorsch, J.R. (2009). Kinetic analysis of late steps of eukaryotic translation initiation. Journal of molecular biology 385, 491-506.

Agirrezabala, X., and Frank, J. (2010). From DNA to proteins via the ribosome: structural insights into the workings of the translation machinery. Human genomics 4, 226-237.

Algire, M.A., Maag, D., and Lorsch, J.R. (2005). Pi release from elF2, not GTP hydrolysis, is the step controlled by start-site selection during eukaryotic translation initiation. Molecular cell 20, 251-262.

Allen, G.S., and Frank, J. (2007). Structural insights on the translation initiation complex: ghosts of a universal initiation complex. Molecular microbiology 63, 941-950.

Allen, G.S., Zavialov, A., Gursky, R., Ehrenberg, M., and Frank, J. (2005). The cryo-EM structure of a translation initiation complex from Escherichia coli. Cell 121, 703-712.

Anderson, J.S., Bretscher, M.S., Clark, B.F., and Marcker, K.A. (1967). A GTP requirement for binding initiator tRNA to ribosomes. Nature 215, 490-492.

Antoun, A., Pavlov, M.Y., Andersson, K., Tenson, T., and Ehrenberg, M. (2003). The roles of initiation factor 2 and guanosine triphosphate in initiation of protein synthesis. The EMBO journal 22, 5593-5601.

Antoun, A., Pavlov, M.Y., Lovmar, M., and Ehrenberg, M. (2006a). How initiation factors maximize the accuracy of tRNA selection in initiation of bacterial protein synthesis. Molecular cell 23, 183-193.

Antoun, A., Pavlov, M.Y., Lovmar, M., and Ehrenberg, M. (2006b). How initiation factors tune the rate of initiation of protein synthesis in bacteria. The EMBO journal 25, 2539-2550.

Antoun, A., Pavlov, M.Y., Tenson, T., and Ehrenberg, M.M. (2004). Ribosome formation from subunits studied by stopped-flow and Rayleigh light scattering. Biological procedures online $6,35-54$.

Bai, X.C., Fernandez, I.S., McMullan, G., and Scheres, S.H. (2013). Ribosome structures to near-atomic resolution from thirty thousand cryo-EM particles. eLife 2, e00461.

Ban, N., Nissen, P., Hansen, J., Moore, P.B., and Steitz, T.A. (2000). The complete atomic structure of the large ribosomal subunit at 2.4 A resolution. Science 289, 905-920.

Beaudry, P., Sander, G., Grunberg-Manago, M., and Douzou, P. (1979). Cation-induced regulatory mechanism of GTPase activity dependent on polypeptide initiation factor 2. Biochemistry 18, 202-207. 
Belotserkovsky, J.M., Dabbs, E.R., and Isaksson, L.A. (2011). Mutations in 16S rRNA that suppress cold-sensitive initiation factor 1 affect ribosomal subunit association. The FEBS journal 278, 3508-3517.

Benne, R., Naaktgeboren, N., Gubbens, J., and Voorma, H.O. (1973). Recycling of initiation factors IF-1, IF-2 and IF-3. European journal of biochemistry / FEBS 32, 372-380.

Blanchard, S.C. (2009). Single-molecule observations of ribosome function. Current opinion in structural biology 19, 103-109.

Blanchard, S.C., Gonzalez, R.L., Kim, H.D., Chu, S., and Puglisi, J.D. (2004). tRNA selection and kinetic proofreading in translation. Nature structural \& molecular biology 11, 1008-1014.

Boelens, R., and Gualerzi, C.O. (2002). Structure and function of bacterial initiation factors. Current protein \& peptide science $3,107-119$.

Boileau, G., Butler, P., Hershey, J.W., and Traut, R.R. (1983). Direct cross-links between initiation factors 1, 2, and 3 and ribosomal proteins promoted by 2-iminothiolane. Biochemistry 22, 3162-3170.

Bradford, M.M. (1976). A rapid and sensitive method for the quantitation of microgram quantities of protein utilizing the principle of protein-dye binding. Analytical biochemistry 72 , 248-254.

Brandt, R., and Gualerzi, C.O. (1991). Ribosome-mRNA contact sites at different stages of translation initiation as revealed by cross-linking of model mRNAs. Biochimie 73, 1543-1549.

Brune, M., Hunter, J.L., Corrie, J.E., and Webb, M.R. (1994). Direct, real-time measurement of rapid inorganic phosphate release using a novel fluorescent probe and its application to actomyosin subfragment 1 ATPase. Biochemistry 33, 8262-8271.

Calogero, R.A., Pon, C.L., Canonaco, M.A., and Gualerzi, C.O. (1988). Selection of the mRNA translation initiation region by Escherichia coli ribosomes. Proceedings of the National Academy of Sciences of the United States of America 85, 6427-6431.

Carter, A.P., Clemons, W.M., Jr., Brodersen, D.E., Morgan-Warren, R.J., Hartsch, T., Wimberly, B.T., and Ramakrishnan, V. (2001). Crystal structure of an initiation factor bound to the 30 S ribosomal subunit. Science $291,498-501$.

Caserta, E., Ferrara, C., Milon, P., Fabbretti, A., Rocchetti, A., Tomsic, J., Pon, C.L., Gualerzi, C.O., and La Teana, A. (2010). Ribosomal interaction of Bacillus stearothermophilus translation initiation factor IF2: characterization of the active sites. Journal of molecular biology 396, 118-129.

Caserta, E., Tomsic, J., Spurio, R., La Teana, A., Pon, C.L., and Gualerzi, C.O. (2006). Translation initiation factor IF2 interacts with the $30 \mathrm{~S}$ ribosomal subunit via two separate binding sites. Journal of molecular biology 362, 787-799.

Celano, B., Pawlik, R.T., and Gualerzi, C.O. (1988). Interaction of Escherichia coli translation-initiation factor IF-1 with ribosomes. European journal of biochemistry / FEBS 178, 351-355. 
Chen, H., Bjerknes, M., Kumar, R., and Jay, E. (1994). Determination of the optimal aligned spacing between the Shine-Dalgarno sequence and the translation initiation codon of Escherichia coli mRNAs. Nucleic acids research 22, 4953-4957.

Croitoru, V., Bucheli-Witschel, M., Hagg, P., Abdulkarim, F., and Isaksson, L.A. (2004). Generation and characterization of functional mutants in the translation initiation factor IF1 of Escherichia coli. European journal of biochemistry / FEBS 271, 534-544.

Crowe, J., Dobeli, H., Gentz, R., Hochuli, E., Stuber, D., and Henco, K. (1994). 6xHis-Ni-NTA chromatography as a superior technique in recombinant protein expression/purification. Methods Mol Biol 31, 371-387.

Cummings, H.S., and Hershey, J.W. (1994). Translation initiation factor IF1 is essential for cell viability in Escherichia coli. Journal of bacteriology 176, 198-205.

Dallas, A., and Noller, H.F. (2001). Interaction of translation initiation factor 3 with the 30 S ribosomal subunit. Molecular cell 8, 855-864.

Davies, D.R., and Hol, W.G. (2004). The power of vanadate in crystallographic investigations of phosphoryl transfer enzymes. FEBS letters 577, 315-321.

Daviter, T., Wieden, H.J., and Rodnina, M.V. (2003). Essential role of histidine 84 in elongation factor Tu for the chemical step of GTP hydrolysis on the ribosome. Journal of molecular biology 332, 689-699.

de Smit, M.H., and van Duin, J. (2003). Translational standby sites: how ribosomes may deal with the rapid folding kinetics of mRNA. Journal of molecular biology 331, 737-743.

Dreyfus, M. (1988). What constitutes the signal for the initiation of protein synthesis on Escherichia coli mRNAs? Journal of molecular biology 204, 79-94.

Eiler, D., Lin, J., Simonetti, A., Klaholz, B.P., and Steitz, T.A. (2013). Initiation factor 2 crystal structure reveals a different domain organization from eukaryotic initiation factor $5 \mathrm{~B}$ and mechanism among translational GTPases. Proceedings of the National Academy of Sciences of the United States of America 110, 15662-15667.

Elvekrog, M.M., and Gonzalez, R.L., Jr. (2013). Conformational selection of translation initiation factor 3 signals proper substrate selection. Nature structural \& molecular biology 20 , 628-633.

Fabbretti, A., Brandi, L., Milon, P., Spurio, R., Pon, C.L., and Gualerzi, C.O. (2012). Translation initiation without IF2-dependent GTP hydrolysis. Nucleic acids research 40, 7946-7955.

Fabbretti, A., Pon, C.L., Hennelly, S.P., Hill, W.E., Lodmell, J.S., and Gualerzi, C.O. (2007). The real-time path of translation factor IF3 onto and off the ribosome. Molecular cell 25, 285296.

Fischer, N., Neumann, P., Konevega, A.L., Bock, L.V., Ficner, R., Rodnina, M.V., and Stark, $\mathrm{H}$. (2015). Structure of the E. coli ribosome-EF-Tu complex at $<3$ A resolution by Cscorrected cryo-EM. Nature 520, 567-570. 
Fraser, C.S., and Doudna, J.A. (2007). Quantitative studies of ribosome conformational dynamics. Quarterly reviews of biophysics 40, 163-189.

Gite, S., Mamaev, S., Olejnik, J., and Rothschild, K. (2000). Ultrasensitive fluorescencebased detection of nascent proteins in gels. Analytical biochemistry 279, 218-225.

Golicnik, M. (2010). Metallic fluoride complexes as phosphate analogues for structural and mechanistic studies of phosphoryl group transfer enzymes. Acta chimica Slovenica 57, 272287.

Goodno, C.C. (1982). Myosin active-site trapping with vanadate ion. Methods in enzymology 85 Pt $B, 116-123$.

Green, R., Switzer, C., and Noller, H.F. (1998). Ribosome-catalyzed peptide-bond formation with an A-site substrate covalently linked to 23 S ribosomal RNA. Science 280, 286-289.

Grigoriadou, C., Marzi, S., Kirillov, S., Gualerzi, C.O., and Cooperman, B.S. (2007a). A quantitative kinetic scheme for $70 \mathrm{~S}$ translation initiation complex formation. Journal of molecular biology $373,562-572$.

Grigoriadou, C., Marzi, S., Pan, D., Gualerzi, C.O., and Cooperman, B.S. (2007b). The translational fidelity function of IF3 during transition from the $30 \mathrm{~S}$ initiation complex to the 70 $S$ initiation complex. Journal of molecular biology 373, 551-561.

Grill, S., Moll, I., Hasenohrl, D., Gualerzi, C.O., and Blasi, U. (2001). Modulation of ribosomal recruitment to 5 '-terminal start codons by translation initiation factors IF2 and IF3. FEBS letters 495, 167-171.

Grunberg-Manago, M., Dessen, P., Pantaloni, D., Godefroy-Colburn, T., Wolfe, A.D., and Dondon, J. (1975). Light-scattering studies showing the effect of initiation factors on the reversible dissociation of Escherichia coli ribosomes. Journal of molecular biology 94, 461478.

Gualerzi, C.O., Brandi, L., Caserta, E., Garofalo, C., Lammi, M., La Teana, A., Petrelli, D., Spurio, R., Tomsic, J., and Pon, C.L. (2001). Initiation factors in the early events of mRNA translation in bacteria. Cold Spring Harbor symposia on quantitative biology 66, 363-376.

Guenneugues, M., Caserta, E., Brandi, L., Spurio, R., Meunier, S., Pon, C.L., Boelens, R., and Gualerzi, C.O. (2000). Mapping the fMet-tRNA(f)(Met) binding site of initiation factor IF2. The EMBO journal 19, 5233-5240.

Harms, J., Schluenzen, F., Zarivach, R., Bashan, A., Gat, S., Agmon, I., Bartels, H., Franceschi, F., and Yonath, A. (2001). High resolution structure of the large ribosomal subunit from a mesophilic eubacterium. Cell 107, 679-688.

Hartz, D., Binkley, J., Hollingsworth, T., and Gold, L. (1990). Domains of initiator tRNA and initiation codon crucial for initiator tRNA selection by Escherichia coli IF3. Genes \& development 4, 1790-1800.

Hauryliuk, V., Mitkevich, V.A., Draycheva, A., Tankov, S., Shyp, V., Ermakov, A., Kulikova, A.A., Makarov, A.A., and Ehrenberg, M. (2009). Thermodynamics of GTP and GDP binding 
to bacterial initiation factor 2 suggests two types of structural transitions. Journal of molecular biology 394, 621-626.

Helgstrand, M., Mandava, C.S., Mulder, F.A., Liljas, A., Sanyal, S., and Akke, M. (2007). The ribosomal stalk binds to translation factors IF2, EF-Tu, EF-G and RF3 via a conserved region of the L12 C-terminal domain. Journal of molecular biology 365, 468-479.

Hennelly, S.P., Antoun, A., Ehrenberg, M., Gualerzi, C.O., Knight, W., Lodmell, J.S., and Hill, W.E. (2005). A time-resolved investigation of ribosomal subunit association. Journal of molecular biology 346, 1243-1258.

Hickerson, R., Majumdar, Z.K., Baucom, A., Clegg, R.M., and Noller, H.F. (2005). Measurement of internal movements within the $30 \mathrm{~S}$ ribosomal subunit using Forster resonance energy transfer. Journal of molecular biology 354, 459-472.

Hirokawa, G., Kiel, M.C., Muto, A., Selmer, M., Raj, V.S., Liljas, A., Igarashi, K., Kaji, H., and Kaji, A. (2002). Post-termination complex disassembly by ribosome recycling factor, a functional tRNA mimic. The EMBO journal 21, 2272-2281.

Holtkamp, W., Cunha, C.E., Peske, F., Konevega, A.L., Wintermeyer, W., and Rodnina, M.V. (2014). GTP hydrolysis by EF-G synchronizes tRNA movement on small and large ribosomal subunits. The EMBO journal 33, 1073-1085.

Huang, C., Mandava, C.S., and Sanyal, S. (2010). The ribosomal stalk plays a key role in IF2-mediated association of the ribosomal subunits. Journal of molecular biology 399, 145153.

Ikemura, T., and Ozeki, H. (1977). Gross map location of Escherichia coli transfer RNA genes. Journal of molecular biology 117, 419-446.

Jackson, R.J., Hellen, C.U., and Pestova, T.V. (2010). The mechanism of eukaryotic translation initiation and principles of its regulation. Nature reviews Molecular cell biology 11 , $113-127$.

Julian, P., Milon, P., Agirrezabala, X., Lasso, G., Gil, D., Rodnina, M.V., and Valle, M. (2011). The Cryo-EM structure of a complete $30 \mathrm{~S}$ translation initiation complex from Escherichia coli. PLoS biology 9, e1001095.

Karimi, R., Pavlov, M.Y., Buckingham, R.H., and Ehrenberg, M. (1999). Novel roles for classical factors at the interface between translation termination and initiation. Molecular cell 3, 601-609.

Kipper, K., Hetenyi, C., Sild, S., Remme, J., and Liiv, A. (2009). Ribosomal intersubunit bridge $\mathrm{B} 2 \mathrm{a}$ is involved in factor-dependent translation initiation and translational processivity. Journal of molecular biology 385, 405-422.

Krafft, C., Diehl, A., Laettig, S., Behlke, J., Heinemann, U., Pon, C.L., Gualerzi, C.O., and Welfle, H. (2000). Interaction of fMet-tRNA(fMet) with the C-terminal domain of translational initiation factor IF2 from Bacillus stearothermophilus. FEBS letters 471, 128-132. 
Kuechler, E. (1971). Role of GTP in the positioning of formylmethionyl-tRNAf on the E. coli ribosome. Nature: New biology 234, 216-218.

Kuhle, B., and Ficner, R. (2014a). elF5B employs a novel domain release mechanism to catalyze ribosomal subunit joining. The EMBO journal 33, 1177-1191.

Kuhle, B., and Ficner, R. (2014b). A monovalent cation acts as structural and catalytic cofactor in translational GTPases. The EMBO journal 33, 2547-2563.

Kycia, J.H., Biou, V., Shu, F., Gerchman, S.E., Graziano, V., and Ramakrishnan, V. (1995). Prokaryotic translation initiation factor IF3 is an elongated protein consisting of two crystallizable domains. Biochemistry 34, 6183-6187.

Kyrpides, N.C., and Woese, C.R. (1998). Universally conserved translation initiation factors. Proceedings of the National Academy of Sciences of the United States of America 95, 224228.

La Teana, A., Gualerzi, C.O., and Brimacombe, R. (1995). From stand-by to decoding site. Adjustment of the mRNA on the $30 \mathrm{~S}$ ribosomal subunit under the influence of the initiation factors. RNA 1, 772-782.

La Teana, A., Gualerzi, C.O., and Dahlberg, A.E. (2001). Initiation factor IF 2 binds to the alpha-sarcin loop and helix 89 of Escherichia coli 23 S ribosomal RNA. RNA 7, 1173-1179.

La Teana, A., Pon, C.L., and Gualerzi, C.O. (1993). Translation of mRNAs with degenerate initiation triplet AUU displays high initiation factor 2 dependence and is subject to initiation factor 3 repression. Proceedings of the National Academy of Sciences of the United States of America 90, 4161-4165.

La Teana, A., Pon, C.L., and Gualerzi, C.O. (1996). Late events in translation initiation. Adjustment of fMet-tRNA in the ribosomal P-site. Journal of molecular biology 256, 667-675.

Laalami, S., Timofeev, A.V., Putzer, H., Leautey, J., and Grunberg-Manago, M. (1994). In vivo study of engineered G-domain mutants of Escherichia coli translation initiation factor IF2. Molecular microbiology 11, 293-302.

Laursen, B.S., Kjaergaard, A.C., Mortensen, K.K., Hoffman, D.W., and Sperling-Petersen, H.U. (2004). The N-terminal domain (IF2N) of bacterial translation initiation factor IF2 is connected to the conserved C-terminal domains by a flexible linker. Protein science : a publication of the Protein Society 13, 230-239.

Laursen, B.S., Mortensen, K.K., Sperling-Petersen, H.U., and Hoffman, D.W. (2003). A conserved structural motif at the $\mathrm{N}$ terminus of bacterial translation initiation factor IF2. The Journal of biological chemistry 278, 16320-16328.

Laursen, B.S., Sorensen, H.P., Mortensen, K.K., and Sperling-Petersen, H.U. (2005). Initiation of protein synthesis in bacteria. Microbiology and molecular biology reviews : MMBR 69, 101-123.

Li, W., Liu, Z., Koripella, R.K., Langlois, R., Sanyal, S., and Frank, J. (2015). Activation of GTP hydrolysis in mRNA-tRNA translocation by elongation factor G. Science advances 1. 
Lockwood, A.H., Sarkar, P., and Maitra, U. (1972). Release of polypeptide chain initiation factor IF-2 during initiation complex formation. Proceedings of the National Academy of Sciences of the United States of America 69, 3602-3605.

Lomakin, I.B., Kolupaeva, V.G., Marintchev, A., Wagner, G., and Pestova, T.V. (2003). Position of eukaryotic initiation factor elF1 on the 40S ribosomal subunit determined by directed hydroxyl radical probing. Genes \& development 17, 2786-2797.

Luchin, S., Putzer, H., Hershey, J.W., Cenatiempo, Y., Grunberg-Manago, M., and Laalami, S. (1999). In vitro study of two dominant inhibitory GTPase mutants of Escherichia coli translation initiation factor IF2. Direct evidence that GTP hydrolysis is necessary for factor recycling. The Journal of biological chemistry 274, 6074-6079.

Ma, J., Campbell, A., and Karlin, S. (2002). Correlations between Shine-Dalgarno sequences and gene features such as predicted expression levels and operon structures. Journal of bacteriology $184,5733-5745$.

Maag, D., Fekete, C.A., Gryczynski, Z., and Lorsch, J.R. (2005). A conformational change in the eukaryotic translation preinitiation complex and release of elF1 signal recognition of the start codon. Molecular cell 17, 265-275.

Maar, D., Liveris, D., Sussman, J.K., Ringquist, S., Moll, I., Heredia, N., Kil, A., Blasi, U., Schwartz, I., and Simons, R.W. (2008). A single mutation in the IF3 N-terminal domain perturbs the fidelity of translation initiation at three levels. Journal of molecular biology 383, 937-944.

MacDougall, D.D., and Gonzalez, R.L., Jr. (2015). Translation initiation factor 3 regulates switching between different modes of ribosomal subunit joining. Journal of molecular biology 427, $1801-1818$.

Mandava, C.S., Peisker, K., Ederth, J., Kumar, R., Ge, X., Szaflarski, W., and Sanyal, S. (2012). Bacterial ribosome requires multiple L12 dimers for efficient initiation and elongation of protein synthesis involving IF2 and EF-G. Nucleic acids research 40, 2054-2064.

Maracci, C., Peske, F., Dannies, E., Pohl, C., and Rodnina, M.V. (2014). Ribosome-induced tuning of GTP hydrolysis by a translational GTPase. Proceedings of the National Academy of Sciences of the United States of America 111, 14418-14423.

Marshall, R.A., Aitken, C.E., and Puglisi, J.D. (2009). GTP hydrolysis by IF2 guides progression of the ribosome into elongation. Molecular cell 35, 37-47.

Marzi, S., Myasnikov, A.G., Serganov, A., Ehresmann, C., Romby, P., Yusupov, M., and Klaholz, B.P. (2007). Structured mRNAs regulate translation initiation by binding to the platform of the ribosome. Cell 130, 1019-1031.

McCutcheon, J.P., Agrawal, R.K., Philips, S.M., Grassucci, R.A., Gerchman, S.E., Clemons, W.M., Jr., Ramakrishnan, V., and Frank, J. (1999). Location of translational initiation factor IF3 on the small ribosomal subunit. Proceedings of the National Academy of Sciences of the United States of America 96, 4301-4306. 
McGinnis, J.L., Liu, Q., Lavender, C.A., Devaraj, A., McClory, S.P., Fredrick, K., and Weeks, K.M. (2015). In-cell SHAPE reveals that free 30 S ribosome subunits are in the inactive state. Proceedings of the National Academy of Sciences of the United States of America 112, 2425-2430.

Meinnel, T., Sacerdot, C., Graffe, M., Blanquet, S., and Springer, M. (1999). Discrimination by Escherichia coli initiation factor IF3 against initiation on non-canonical codons relies on complementarity rules. Journal of molecular biology $290,825-837$.

Meunier, S., Spurio, R., Czisch, M., Wechselberger, R., Guenneugues, M., Gualerzi, C.O., and Boelens, R. (2000). Structure of the fMet-tRNA(fMet)-binding domain of B. stearothermophilus initiation factor IF2. The EMBO journal 19, 1918-1926.

Milon, P., Carotti, M., Konevega, A.L., Wintermeyer, W., Rodnina, M.V., and Gualerzi, C.O. (2010). The ribosome-bound initiation factor 2 recruits initiator tRNA to the 30S initiation complex. EMBO reports 11, 312-316.

Milon, P., Konevega, A.L., Gualerzi, C.O., and Rodnina, M.V. (2008). Kinetic checkpoint at a late step in translation initiation. Molecular cell 30, 712-720.

Milon, P., Konevega, A.L., Peske, F., Fabbretti, A., Gualerzi, C.O., and Rodnina, M.V. (2007). Transient kinetics, fluorescence, and FRET in studies of initiation of translation in bacteria. Methods in enzymology 430, 1-30.

Milon, P., Maracci, C., Filonava, L., Gualerzi, C.O., and Rodnina, M.V. (2012). Real-time assembly landscape of bacterial $30 \mathrm{~S}$ translation initiation complex. Nature structural \& molecular biology 19, 609-615.

Milon, P., and Rodnina, M.V. (2012). Kinetic control of translation initiation in bacteria. Critical reviews in biochemistry and molecular biology 47, 334-348.

Milon, P., Tischenko, E., Tomsic, J., Caserta, E., Folkers, G., La Teana, A., Rodnina, M.V., Pon, C.L., Boelens, R., and Gualerzi, C.O. (2006). The nucleotide-binding site of bacterial translation initiation factor 2 (IF2) as a metabolic sensor. Proceedings of the National Academy of Sciences of the United States of America 103, 13962-13967.

Moazed, D., Samaha, R.R., Gualerzi, C., and Noller, H.F. (1995). Specific protection of 16 S rRNA by translational initiation factors. Journal of molecular biology 248, 207-210.

Moll, I., Hirokawa, G., Kiel, M.C., Kaji, A., and Blasi, U. (2004). Translation initiation with 70 S ribosomes: an alternative pathway for leaderless mRNAs. Nucleic acids research 32, 33543363.

Moreau, M., de Cock, E., Fortier, P.L., Garcia, C., Albaret, C., Blanquet, S., Lallemand, J.Y., and Dardel, F. (1997). Heteronuclear NMR studies of E. coli translation initiation factor IF3. Evidence that the inter-domain region is disordered in solution. Journal of molecular biology 266, 15-22.

Moreno, J.M., Drskjotersen, L., Kristensen, J.E., Mortensen, K.K., and Sperling-Petersen, H.U. (1999). Characterization of the domains of E. coli initiation factor IF2 responsible for recognition of the ribosome. FEBS letters 455, 130-134. 
Mortensen, K.K., Kildsgaard, J., Moreno, J.M., Steffensen, S.A., Egebjerg, J., and SperlingPetersen, H.U. (1998). A six-domain structural model for Escherichia coli translation initiation factor IF2. Characterisation of twelve surface epitopes. Biochemistry and molecular biology international 46, 1027-1041.

Mullis, K., Faloona, F., Scharf, S., Saiki, R., Horn, G., and Erlich, H. (1986). Specific enzymatic amplification of DNA in vitro: the polymerase chain reaction. Cold Spring Harbor symposia on quantitative biology 51 Pt 1, 263-273.

Myasnikov, A.G., Marzi, S., Simonetti, A., Giuliodori, A.M., Gualerzi, C.O., Yusupova, G., Yusupov, M., and Klaholz, B.P. (2005). Conformational transition of initiation factor 2 from the GTP- to GDP-bound state visualized on the ribosome. Nature structural \& molecular biology $12,1145-1149$.

Myasnikov, A.G., Simonetti, A., Marzi, S., and Klaholz, B.P. (2009). Structure-function insights into prokaryotic and eukaryotic translation initiation. Current opinion in structural biology 19, 300-309.

Paleskava, A., Konevega, A.L., and Rodnina, M.V. (2012). Thermodynamics of the GTPGDP-operated conformational switch of selenocysteine-specific translation factor SelB. The Journal of biological chemistry $287,27906-27912$.

Panico, J., Parkes, J.H., and Liebman, P.A. (1990). The effect of GDP on rod outer segment G-protein interactions. The Journal of biological chemistry 265, 18922-18927.

Pape, T., Wintermeyer, W., and Rodnina, M.V. (1998). Complete kinetic mechanism of elongation factor Tu-dependent binding of aminoacyl-tRNA to the A site of the E. coli ribosome. The EMBO journal 17, 7490-7497.

Pavlov, M.Y., Zorzet, A., Andersson, D.I., and Ehrenberg, M. (2011). Activation of initiation factor 2 by ligands and mutations for rapid docking of ribosomal subunits. The EMBO journal 30, 289-301.

Peske, F., Rodnina, M.V., and Wintermeyer, W. (2005). Sequence of steps in ribosome recycling as defined by kinetic analysis. Molecular cell 18, 403-412.

Pestova, T.V., Lomakin, I.B., Lee, J.H., Choi, S.K., Dever, T.E., and Hellen, C.U. (2000). The joining of ribosomal subunits in eukaryotes requires elF5B. Nature 403, 332-335.

Petrelli, D., LaTeana, A., Garofalo, C., Spurio, R., Pon, C.L., and Gualerzi, C.O. (2001). Translation initiation factor IF3: two domains, five functions, one mechanism? The EMBO journal 20, 4560-4569.

Petrov, A., Chen, J., O'Leary, S., Tsai, A., and Puglisi, J.D. (2012). Single-molecule analysis of translational dynamics. Cold Spring Harbor perspectives in biology 4, a011551.

Pioletti, M., Schlunzen, F., Harms, J., Zarivach, R., Gluhmann, M., Avila, H., Bashan, A., Bartels, H., Auerbach, T., Jacobi, C., et al. (2001). Crystal structures of complexes of the small ribosomal subunit with tetracycline, edeine and IF3. The EMBO journal 20, 1829-1839. 
Pisarev, A.V., Unbehaun, A., Hellen, C.U., and Pestova, T.V. (2007). Assembly and analysis of eukaryotic translation initiation complexes. Methods in enzymology 430, 147-177.

Pon, C.L., and Gualerzi, C.O. (1984). Mechanism of protein biosynthesis in prokaryotic cells. Effect of initiation factor IF1 on the initial rate of $30 \mathrm{~S}$ initiation complex formation. FEBS letters 175, 203-207.

Pon, C.L., and Gualerzi, C.O. (1986). Mechanism of translational initiation in prokaryotes. IF3 is released from ribosomes during and not before $70 \mathrm{~S}$ initiation complex formation. FEBS letters 195, 215-219.

Qin, D., and Fredrick, K. (2009). Control of translation initiation involves a factor-induced rearrangement of helix 44 of $16 \mathrm{~S}$ ribosomal RNA. Molecular microbiology 71, 1239-1249.

Qin, D., Liu, Q., Devaraj, A., and Fredrick, K. (2012). Role of helix 44 of $16 \mathrm{~S}$ rRNA in the fidelity of translation initiation. RNA $18,485-495$.

Qin, H., Grigoriadou, C., and Cooperman, B.S. (2009). Interaction of IF2 with the ribosomal GTPase-associated center during $70 S$ initiation complex formation. Biochemistry 48, 46994706.

Ramakrishnan, V. (2002). Ribosome structure and the mechanism of translation. Cell 108, 557-572.

Rasmussen, L.C., Oliveira, C.L., Jensen, J.M., Pedersen, J.S., Sperling-Petersen, H.U., and Mortensen, K.K. (2008). Solution structure of C-terminal Escherichia coli translation initiation factor IF2 by small-angle X-ray scattering. Biochemistry 47, 5590-5598.

Rodnina, M.V., Beringer, M., and Wintermeyer, W. (2006). Mechanism of peptide bond formation on the ribosome. Quarterly reviews of biophysics 39, 203-225.

Rodnina, M.V., Fricke, R., and Wintermeyer, W. (1994). Transient conformational states of aminoacyl-tRNA during ribosome binding catalyzed by elongation factor Tu. Biochemistry 33, 12267-12275.

Rodnina, M.V., Savelsbergh, A., Katunin, V.I., and Wintermeyer, W. (1997). Hydrolysis of GTP by elongation factor G drives tRNA movement on the ribosome. Nature 385, 37-41.

Rodnina, M.V., and Wintermeyer, W. (1995). GTP consumption of elongation factor Tu during translation of heteropolymeric mRNAs. Proceedings of the National Academy of Sciences of the United States of America 92, 1945-1949.

Roll-Mecak, A., Cao, C., Dever, T.E., and Burley, S.K. (2000). X-Ray structures of the universal translation initiation factor IF2/elF5B: conformational changes on GDP and GTP binding. Cell 103, 781-792.

Savelsbergh, A., Katunin, V.I., Mohr, D., Peske, F., Rodnina, M.V., and Wintermeyer, W. (2003). An elongation factor G-induced ribosome rearrangement precedes tRNA-mRNA translocation. Molecular cell 11, 1517-1523. 
Schluenzen, F., Tocilj, A., Zarivach, R., Harms, J., Gluehmann, M., Janell, D., Bashan, A., Bartels, H., Agmon, I., Franceschi, F., et al. (2000). Structure of functionally activated small ribosomal subunit at 3.3 angstroms resolution. Cell 102, 615-623.

Schmeing, T.M., and Ramakrishnan, V. (2009). What recent ribosome structures have revealed about the mechanism of translation. Nature 461, 1234-1242.

Schuwirth, B.S., Borovinskaya, M.A., Hau, C.W., Zhang, W., Vila-Sanjurjo, A., Holton, J.M., and Cate, J.H. (2005). Structures of the bacterial ribosome at 3.5 A resolution. Science 310, 827-834.

Selmer, M., Dunham, C.M., Murphy, F.V.t., Weixlbaumer, A., Petry, S., Kelley, A.C., Weir, J.R., and Ramakrishnan, V. (2006). Structure of the 70 S ribosome complexed with mRNA and tRNA. Science 313, 1935-1942.

Sette, M., Spurio, R., van Tilborg, P., Gualerzi, C.O., and Boelens, R. (1999). Identification of the ribosome binding sites of translation initiation factor IF3 by multidimensional heteronuclear NMR spectroscopy. RNA 5, 82-92.

Severini, M., Spurio, R., La Teana, A., Pon, C.L., and Gualerzi, C.O. (1991). Ribosomeindependent GTPase activity of translation initiation factor IF2 and of its G-domain. The Journal of biological chemistry 266, 22800-22802.

Shapiro, A.L., Vinuela, E., and Maizel, J.V., Jr. (1967). Molecular weight estimation of polypeptide chains by electrophoresis in SDS-polyacrylamide gels. Biochemical and biophysical research communications $28,815-820$.

Shapkina, T.G., Dolan, M.A., Babin, P., and Wollenzien, P. (2000). Initiation factor 3-induced structural changes in the $30 \mathrm{~S}$ ribosomal subunit and in complexes containing tRNA(f)(Met) and mRNA. Journal of molecular biology 299, 615-628.

Shin, B.S., Maag, D., Roll-Mecak, A., Arefin, M.S., Burley, S.K., Lorsch, J.R., and Dever, T.E. (2002). Uncoupling of initiation factor elF5B/IF2 GTPase and translational activities by mutations that lower ribosome affinity. Cell 111, 1015-1025.

Shine, J., and Dalgarno, L. (1974). The 3'-terminal sequence of Escherichia coli $16 \mathrm{~S}$ ribosomal RNA: complementarity to nonsense triplets and ribosome binding sites. Proceedings of the National Academy of Sciences of the United States of America 71, 13421346.

Shoji, S., Abdi, N.M., Bundschuh, R., and Fredrick, K. (2009). Contribution of ribosomal residues to P-site tRNA binding. Nucleic acids research 37, 4033-4042.

Simonetti, A., Marzi, S., Fabbretti, A., Hazemann, I., Jenner, L., Urzhumtsev, A., Gualerzi, C.O., and Klaholz, B.P. (2013). Structure of the protein core of translation initiation factor 2 in apo, GTP-bound and GDP-bound forms. Acta crystallographica Section D, Biological crystallography 69, 925-933.

Simonetti, A., Marzi, S., Jenner, L., Myasnikov, A., Romby, P., Yusupova, G., Klaholz, B.P., and Yusupov, M. (2009). A structural view of translation initiation in bacteria. Cellular and molecular life sciences : CMLS 66, 423-436. 
Simonetti, A., Marzi, S., Myasnikov, A.G., Fabbretti, A., Yusupov, M., Gualerzi, C.O., and Klaholz, B.P. (2008). Structure of the 30 S translation initiation complex. Nature 455, 416-420.

Spurio, R., Brandi, L., Caserta, E., Pon, C.L., Gualerzi, C.O., Misselwitz, R., Krafft, C., Welfle, K., and Welfle, H. (2000). The C-terminal subdomain (IF2 C-2) contains the entire fMet-tRNA binding site of initiation factor IF2. The Journal of biological chemistry 275, 2447-2454.

Stringer, E.A., Sarkar, P., and Maitra, U. (1977). Function of initiation factor 1 in the binding and release of initiation factor 2 from ribosomal initiation complexes in Escherichia coli. The Journal of biological chemistry 252, 1739-1744.

Studer, S.M., and Joseph, S. (2006). Unfolding of mRNA secondary structure by the bacterial translation initiation complex. Molecular cell 22, 105-115.

Sun, C., Todorovic, A., Querol-Audi, J., Bai, Y., Villa, N., Snyder, M., Ashchyan, J., Lewis, C.S., Hartland, A., Gradia, S., et al. (2011). Functional reconstitution of human eukaryotic translation initiation factor 3 (elF3). Proceedings of the National Academy of Sciences of the United States of America 108, 20473-20478.

Suragani, R.N., Ghosh, S., Ehtesham, N.Z., and Ramaiah, K.V. (2006). Expression and purification of the subunits of human translational initiation factor 2 (elF2): phosphorylation of elF2 alpha and beta. Protein expression and purification 47, 225-233.

Surkov, S., Nilsson, H., Rasmussen, L.C., Sperling-Petersen, H.U., and Isaksson, L.A. (2010). Translation initiation region dependency of translation initiation in Escherichia coli by IF1 and kasugamycin. The FEBS journal 277, 2428-2439.

Sussman, J.K., Simons, E.L., and Simons, R.W. (1996). Escherichia coli translation initiation factor 3 discriminates the initiation codon in vivo. Molecular microbiology 21, 347-360.

Thach, S.S., and Thach, R.E. (1971). Translocation of messenger RNA and "accommodation" of fMet-tRNA. Proceedings of the National Academy of Sciences of the United States of America 68, 1791-1795.

Tomsic, J., Vitali, L.A., Daviter, T., Savelsbergh, A., Spurio, R., Striebeck, P., Wintermeyer, W., Rodnina, M.V., and Gualerzi, C.O. (2000). Late events of translation initiation in bacteria: a kinetic analysis. The EMBO journal 19, 2127-2136.

Tsai, A., Petrov, A., Marshall, R.A., Korlach, J., Uemura, S., and Puglisi, J.D. (2012). Heterogeneous pathways and timing of factor departure during translation initiation. Nature 487, 390-393.

Wang, J., Caban, K., and Gonzalez, R.L., Jr. (2015). Ribosomal initiation complex-driven changes in the stability and dynamics of initiation factor 2 regulate the fidelity of translation initiation. Journal of molecular biology 427, 1819-1834.

Weiel, J., and Hershey, J.W. (1982). The binding of fluorescein-labeled protein synthesis initiation factor 2 to Escherichia coli $30 \mathrm{~S}$ ribosomal subunits determined by fluorescence polarization. The Journal of biological chemistry 257, 1215-1220. 
Wienk, H., Tishchenko, E., Belardinelli, R., Tomaselli, S., Dongre, R., Spurio, R., Folkers, G.E., Gualerzi, C.O., and Boelens, R. (2012). Structural dynamics of bacterial translation initiation factor IF2. The Journal of biological chemistry 287, 10922-10932.

Wimberly, B.T., Brodersen, D.E., Clemons, W.M., Jr., Morgan-Warren, R.J., Carter, A.P., Vonrhein, C., Hartsch, T., and Ramakrishnan, V. (2000). Structure of the 30 S ribosomal subunit. Nature 407, 327-339.

Wintermeyer, W., Peske, F., Beringer, M., Gromadski, K.B., Savelsbergh, A., and Rodnina, M.V. (2004). Mechanisms of elongation on the ribosome: dynamics of a macromolecular machine. Biochemical Society transactions 32, 733-737.

Wishnia, A., Boussert, A., Graffe, M., Dessen, P.H., and Grunberg-Manago, M. (1975). Kinetics of the reversible association of ribosomal subunits: stopped-flow studies of the rate law and of the effect of Mg2+. Journal of molecular biology 93, 499-415.

Yusupov, M.M., Yusupova, G.Z., Baucom, A., Lieberman, K., Earnest, T.N., Cate, J.H., and Noller, H.F. (2001). Crystal structure of the ribosome at 5.5 A resolution. Science 292, 883896.

Yusupova, G., Jenner, L., Rees, B., Moras, D., and Yusupov, M. (2006). Structural basis for messenger RNA movement on the ribosome. Nature 444, 391-394.

Yusupova, G.Z., Yusupov, M.M., Cate, J.H., and Noller, H.F. (2001). The path of messenger RNA through the ribosome. Cell 106, 233-241.

Zoldak, G., Sedlak, E., Wolfrum, A., Musatov, A., Fedunova, D., Szkaradkiewicz, K., and Sprinzl, M. (2008). Multidomain initiation factor 2 from Thermus thermophilus consists of the individual autonomous domains. Biochemistry 47, 4992-5005.

Zorzet, A., Pavlov, M.Y., Nilsson, A.I., Ehrenberg, M., and Andersson, D.I. (2010). Errorprone initiation factor 2 mutations reduce the fitness cost of antibiotic resistance. Molecular microbiology 75, 1299-1313.

Zucker, F.H., and Hershey, J.W. (1986). Binding of Escherichia coli protein synthesis initiation factor IF1 to $30 \mathrm{~S}$ ribosomal subunits measured by fluorescence polarization. Biochemistry 25, 3682-3690. 


\section{LIST OF ABBREVIATIONS}

\begin{tabular}{|c|c|c|}
\hline $30 \mathrm{~S} \mathrm{IC}$ & - & 30S Initiation Complex \\
\hline 30S PIC & - & 30S Pre-Initiation Complex \\
\hline $70 S \mathrm{EC}$ & - & 70S Elongation Complex \\
\hline $70 \mathrm{~S} \mathrm{IC}$ & - & 70S Initiation Complex \\
\hline 70 S IC (Bpy) & - & $70 S$ IC containing Bpy-Met-tRNA ${ }^{\text {fMet }}$ \\
\hline 70S PIC & - & 70S Pre-Initiation Complex \\
\hline$\mu \mathrm{M}$ & - & micromolar \\
\hline A site & - & Aminoacyl site \\
\hline$A_{600}$ & - & Absorbance measured at $600 \mathrm{~nm}$ \\
\hline aa-tRNA ${ }^{\text {aa }}$ & - & aminoacyl-tRNA \\
\hline $\mathrm{AlF}_{3}$ & - & aluminium fluoride \\
\hline Alx & - & Alexa dye \\
\hline $\mathrm{BeF}_{3}$ & - & beryllium fluoride \\
\hline Bpy & - & Bodipy FL \\
\hline Cryo - EM & - & Cryo - Electron Microscopy \\
\hline CTD & - & C-terminal domain \\
\hline cys & - & cysteine \\
\hline dark & - & non-fluorescent \\
\hline dc & - & decoding center \\
\hline D-stem & - & Dihydrouridine stem \\
\hline E site & - & Exit site \\
\hline EF & - & Elongation Factor \\
\hline EF-Tu TC & - & EF-Tu_GTP_Phe-tRNA ${ }^{\text {Phe }}$ Ternary Complex \\
\hline f-Met & - & formyl-methionine \\
\hline FPLC & - & Fast Protein Liquid Chromatography \\
\hline IF & - & Initiation Factor \\
\hline$k_{\text {app }}$ & - & Apparent rate constant \\
\hline $\mathrm{K}_{\mathrm{d}}$ & - & Dissociation rate constant \\
\hline LS & - & Light Scattering \\
\hline mRNA & - & messenger RNA \\
\hline n.d. & - & not determined \\
\hline n.o. & - & not observed \\
\hline
\end{tabular}




$\begin{array}{lll}\text { Ni-NTA } & - & \text { Nickel- nitrile triacetic acid } \\ \text { nm } & - & \text { nanometer } \\ \text { NTD } & - & \text { N-terminal domain } \\ \text { OB } & - & \text { Oligonucleotide-binding domain } \\ \text { P site } & - & \text { Peptidyl site } \\ \text { P/l site } & - & \text { Peptidyl/intermediate site } \\ \text { PCR } & - & \text { Polymerase Chain Reaction } \\ \text { Pi } & - & \text { Inorganic Phosphate } \\ \text { PTC } & - & \text { Peptidyl Transferase Center } \\ \text { RF } & - & \text { Release Factor } \\ \text { RRF } & - & \text { Ribosomal Release Factor } \\ \text { rRNA } & - & \text { ribosomal RNA } \\ \text { SD } & - & \text { Shine-Dalgarno sequence } \\ \text { SDS-PAGE } & - & \text { Sodium dodecyl sulphate- polyacrylamide gel } \\ & - & \text { electrophoresis } \\ \text { TIR } & - & \text { Translation Initiation Region } \\ \text { tRNA } & - & \text { transfer RNA } \\ \text { tRNA (Flu) } & - & \text { Fluorescein-labeled fMet-tRNA } \\ \text { V/v } & - & \text { volumel } \\ \text { wt } & - & \text { wild type }\end{array}$




\section{ACKNOWLEDGEMENTS}

"There is no path to happiness. Happiness is the path." Unknown

Wow, it's almost over. Four and a half years - they passed by so quickly. My PhD was, like most phDs, interspersed with highs and lows. And every step forward was taken with the support of kind and generous individuals whom I would like to sincerely thank.

First and foremost, I thank Prof. Marina Rodnina, Boehringer Ingelheim Fonds and Dr. Steffen Burkhardt. I am proudly here today, able to submit this PhD thesis because they believed in me. Not just in my scientific potential, but more importantly in me as a person. They undoubtedly played a tremendous role in guiding my studies, but were also there for me during the hard times when I was temporarily unable to work - and for that I am immensely grateful. I thank Prof. Wintermeyer and my thesis committee members, Prof. Neumann and Prof. Krebber for their support, career advice and very helpful suggestions about the PhD project.

I still remember my lab rotation in Marina's department - it was the most fun, and it introduced me to another important guide - Dr. Pohl Milon. Pohl played a very important role in shaping me as an experimentalist. He made rapid kinetics sound so fantastic and exciting, and it really was. In a way, the project was his brain-child and I was fortunate enough to care for it. Then Dr. Riccardo Belardinelli and Dr. Cristina Maracci joined us with their fluorescent components and excellent ideas. Under Marina's guidance and backed by a team of competent technical staff - Sandra, Olaf, Christina K and Tanja - we managed to produce results which were novel and relevant in a field that has been mostly ignored for the last 7 years.

My PhD was not only about bacterial initiation - I dabbled with eukaryotic initiation as well. With help from Christina K, Prof. Tatyana Pestova and her super helpful lab members, we managed to establish in our lab the rather complex, reconstituted system of mammalian initiation, which consists of at least 15 components. Currently, we are using the system as a prelude to study translation elongation (with Dr. Dmitry Burakovsky) and will soon progress to the termination stage.

Of course, work would not have been nearly as enjoyable were it not for my wonderful colleagues and friends. I am thankful for the joy, laughter and great conversations I had with Michael, Heena, Neva, Raffaela, Irena, Carlos, Karine, Dimitra and Sejeong. And my two favorite girls in the world - Veena and Chaitali. The three of us have been together since the 
day we first landed in Germany. We have stood by each other every step of the way and I cannot believe that soon we will head towards our different destinations. I will really miss you both.

And now for my lifeline - my family. Dad, you inspired me since I was a child to work hard and be curious. You set the bar and I have always strived to achieve it and make you proud. Mom, you have made me the person that I am today. You have instilled in me your stability and virtues that have helped me find happiness and peace. You are my best friend. My sister, my angel Manu, you are the kindest person I have ever known and it makes me so happy to see you now with your beautiful family. And finally, my husband Eike. I met you a week before I started my $\mathrm{PhD}$ and you have been there with me, for me, every day since. Before we got married we had already fulfilled the traditional vows - in richness and poor, in sickness and in health - and now I look forward to spending the rest of my life by your side. 


\section{Curriculum Vitae}

\section{Personal Information}

$\begin{array}{ll}\text { Name } & \text { Akanksha Goyal } \\ \text { Email id: } & \text { akanksha.goyal@mpibpc.mpg.de } \\ \text { Date of Birth } & 22.11 .1988 \\ \text { Nationality } & \text { Indian }\end{array}$

\section{Education}

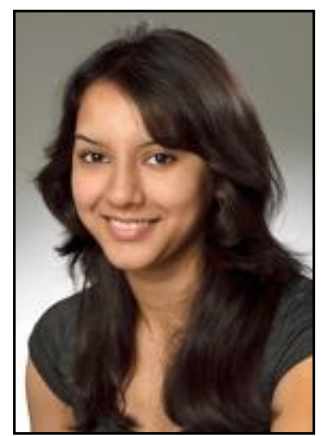

\begin{tabular}{|c|c|}
\hline 2011 - present & Ph.D in Molecular Biology \\
\hline & Max Planck Institute for Biophysical Chemistry, Göttingen, Germany \\
\hline $2009-2011$ & M.Sc in Molecular Biology \\
\hline & International Max Planck Research School (IMPRS) for Molecular \\
\hline & Biology, Göttingen, Germany \\
\hline & Grade $-1.0($ scale $1.0-4.0)=A($ scale $A-F)$ \\
\hline $2006-2009$ & B.Sc (Honors) in Biochemistry \\
\hline & Sri Venkateswara College, University of Delhi, New Delhi, India \\
\hline & Grade $-81.7 \%$ (scale $0-100 \%)$ \\
\hline
\end{tabular}

\section{Fellowships}

2012 - $2014 \quad$ Boehringer Ingelheim Fonds PhD Fellowship

$2011-2012 \quad$ GGNB Excellence Stipend

$2009-2010 \quad$ Stipend of the Excellence Foundation for the promotion of the Max Planck Society

\section{Publications}

Goyal, A., Belardinelli, R., Maracci, C., Milón, P., Rodnina, M.V. Directional transition from initiation to elongation in bacterial translation. Nucleic Acids Research (2015) 
